Aus der Sportmedizin

(Prof. Dr. med., Dr. rer. nat. A. Niklas)

Schwerpunkt Rehabilitations- und Sportmedizin

der Medizinischen Fakultät der Universität Göttingen

\title{
Trainingsbegleitende Leistungsdiagnostik im Behindertensport Ski alpin
}

\author{
INAUGURAL - DISSERTATION \\ zur Erlangung des Doktorgrades \\ der Medizinischen Fakultät der \\ Georg-August-Universität zu Göttingen \\ vorgelegt von \\ Friederike Wippermann \\ aus \\ Hamburg
}

Göttingen 2011 
Dekan: $\quad$ Prof. Dr. med. C. Frömmel

I. Berichterstatter: Prof. Dr. med. et Dr. rer. nat. A. Niklas

II. Berichterstatter: Priv.-Doz. Dr. med. R. Wachter

III. Berichterstatter: Prof. Dr. med. et Dr. rer. nat. Th. Crozier

Tag der mündlichen Prüfung: 05.10.2011 


\section{Inhaltsverzeichnis}

1 Einleitung. .1

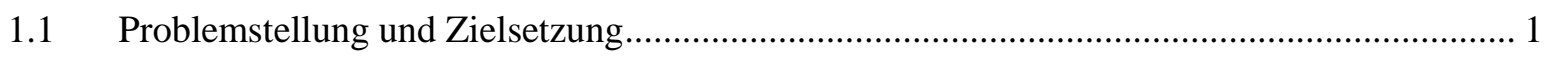

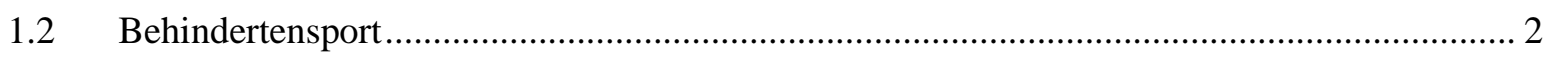

1.2.1 Definition Behinderung und Behindertensport........................................................ 2

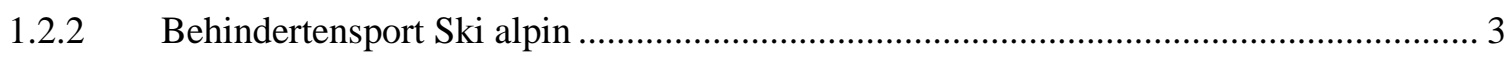

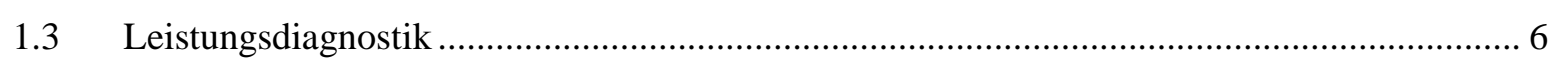

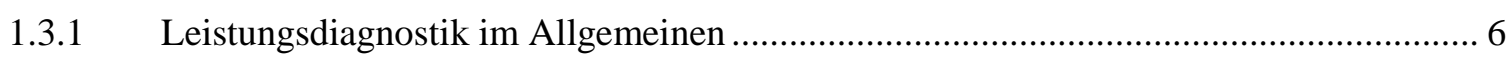

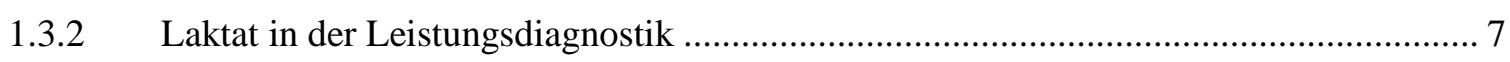

1.3.3 Besonderheiten der Leistungsdiagnostik im Behindertensport ................................... 13

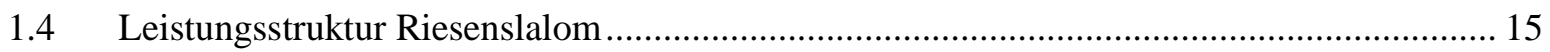

1.5 Leistungsdiagnostische Untersuchungen im Skisport alpin ............................................. 20

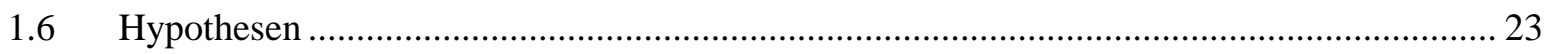

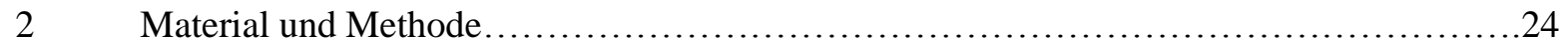

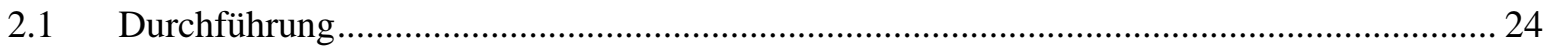

2.2 Vorstellung der einzelnen Athleten und Behinderungen ................................................... 26

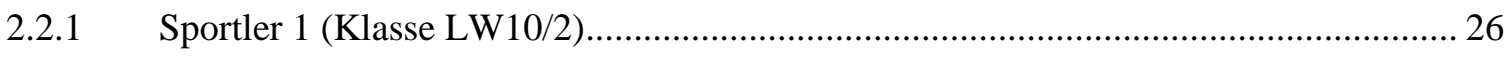

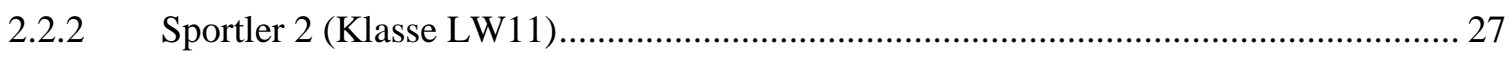

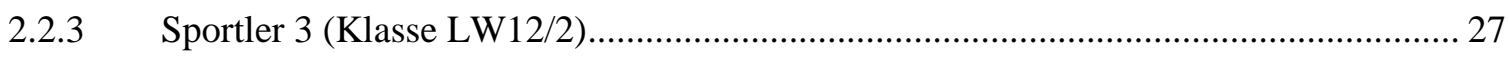

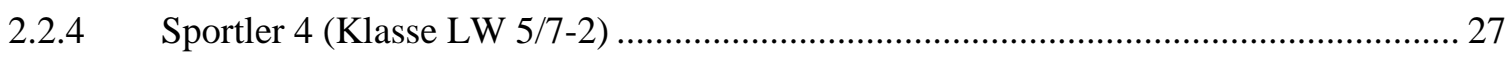

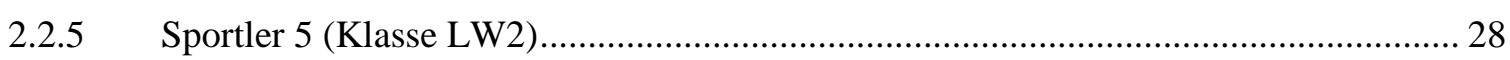

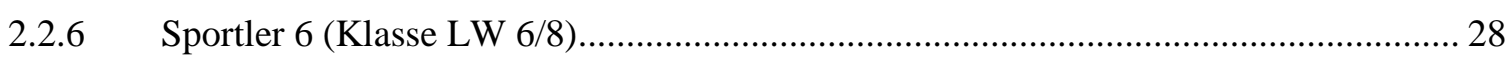

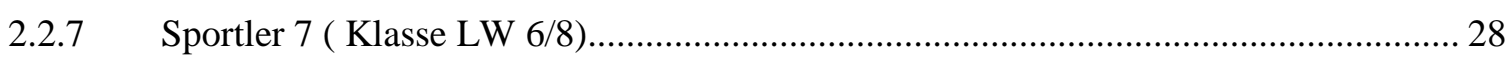

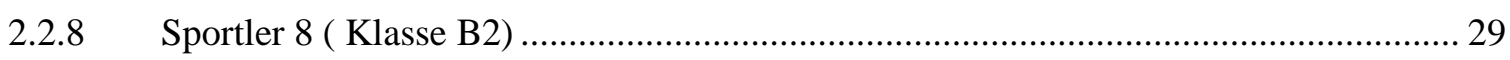

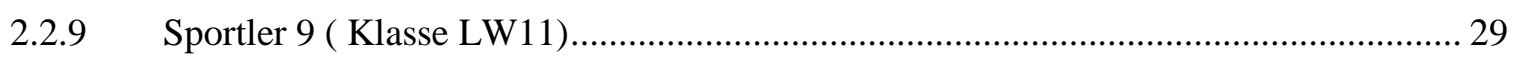

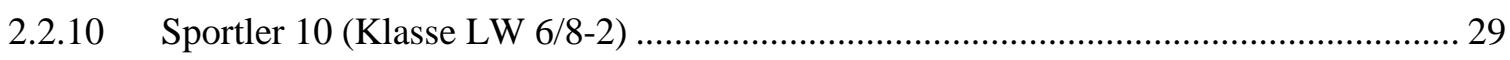

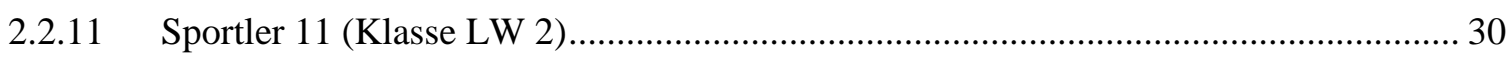

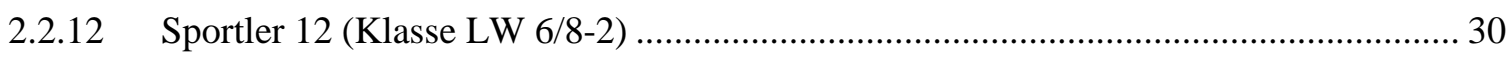

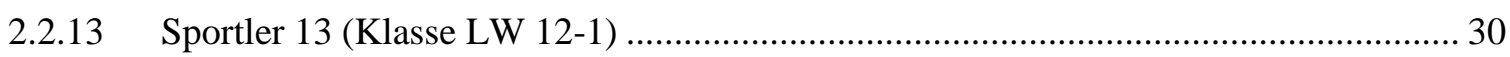

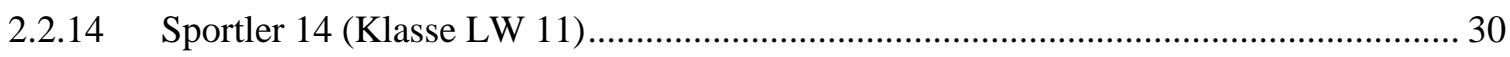

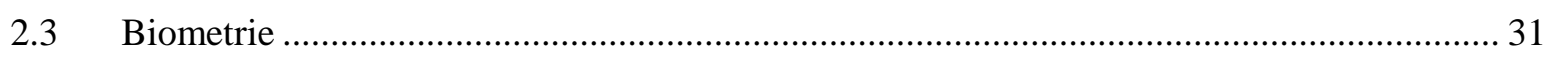

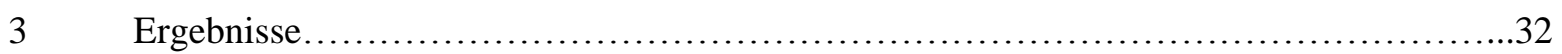

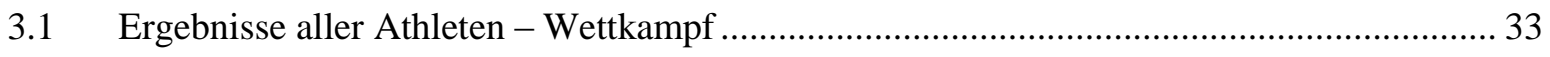

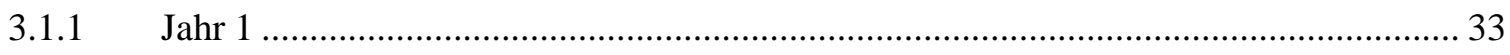

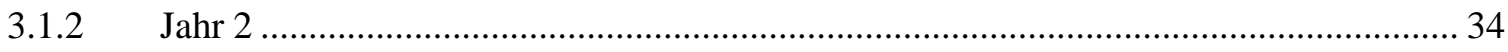




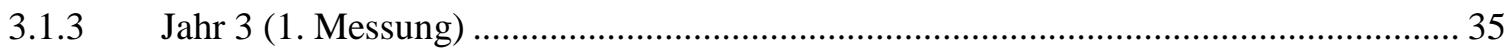

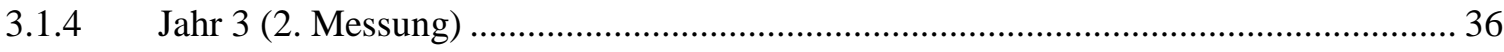

3.2 Sitzende und stehende Sportler - Einzelfallbetrachtung .................................................... 38

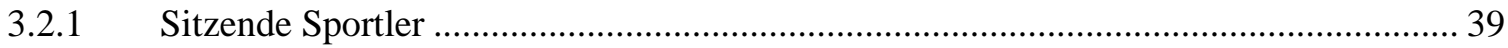

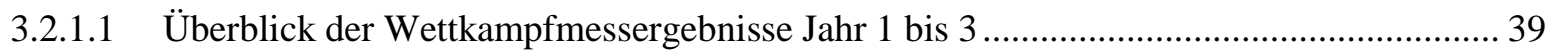

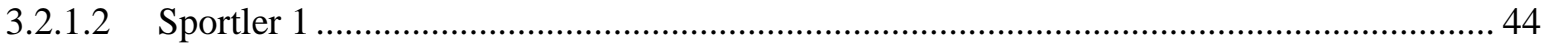

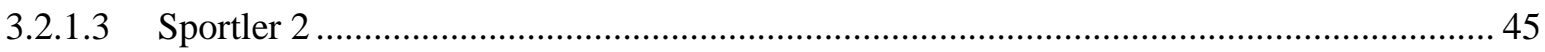

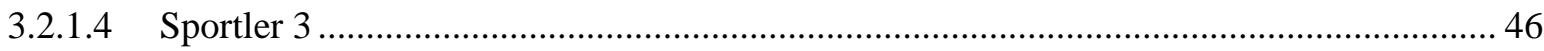

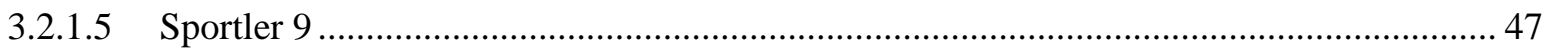

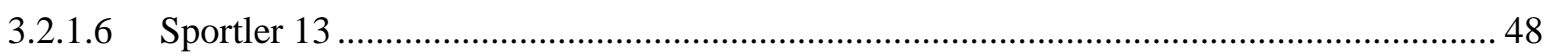

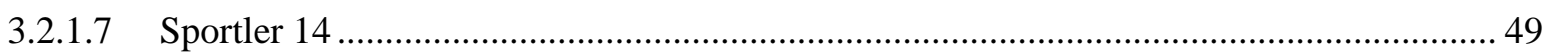

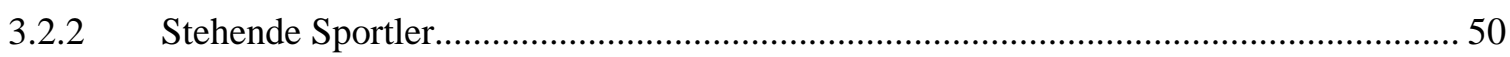

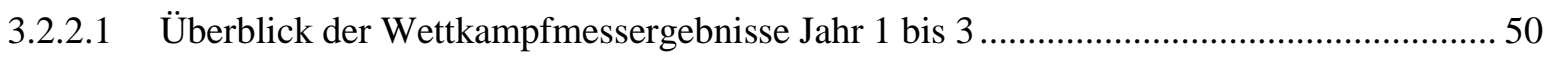

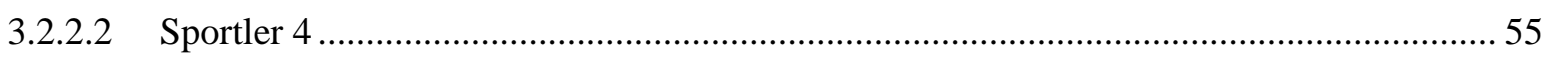

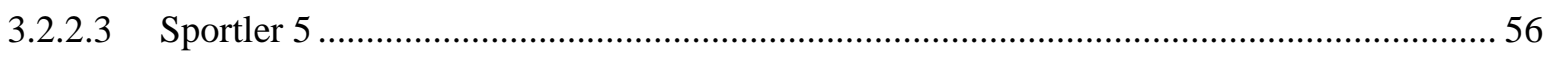

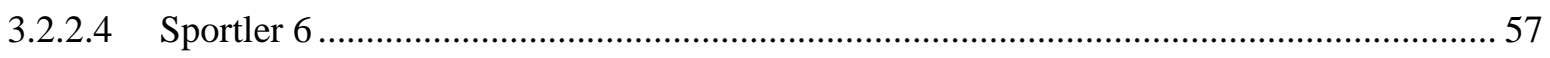

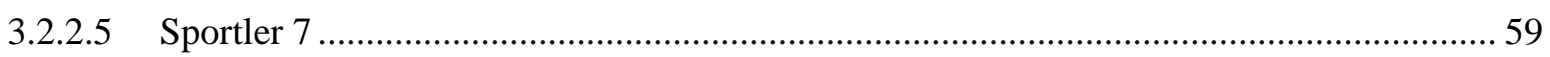

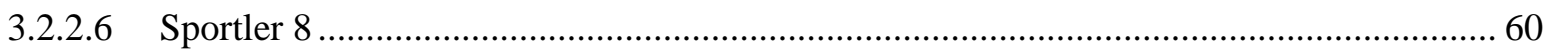

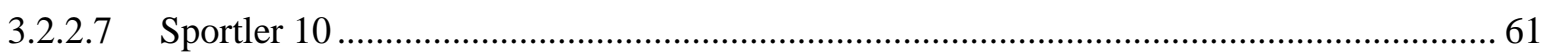

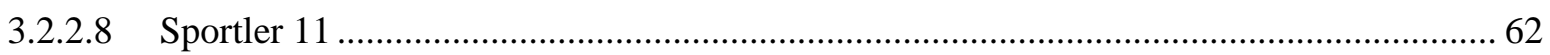

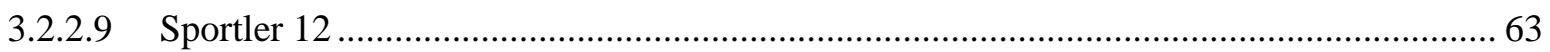

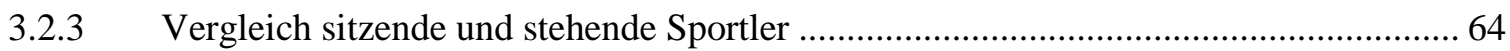

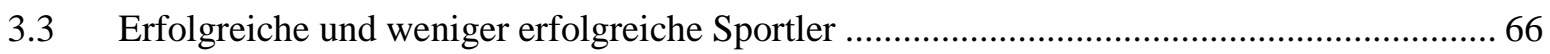

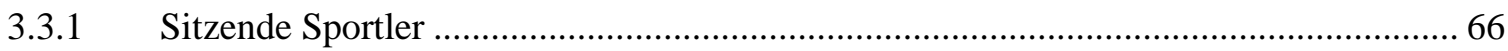

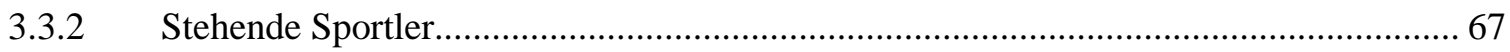

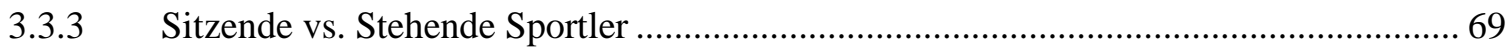

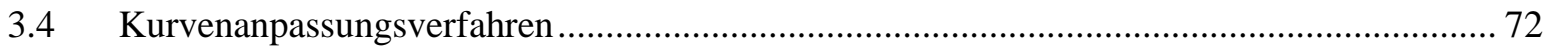

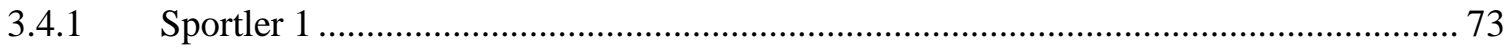

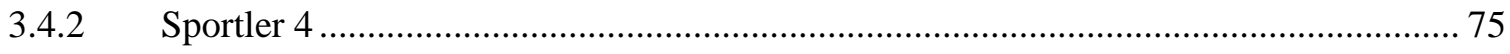

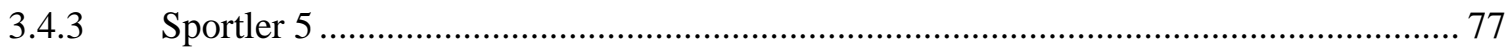

3.5 Vergleich Athleten Behindertensport und gesunde Vergleichsgruppe ................................. 79

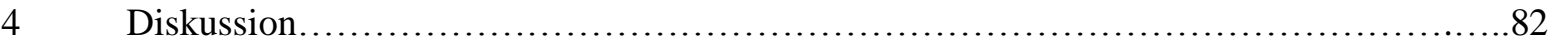

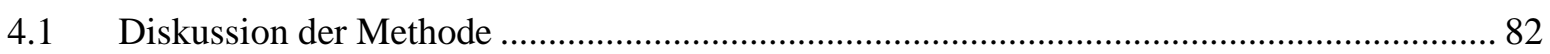

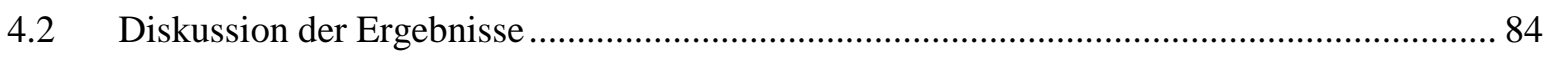

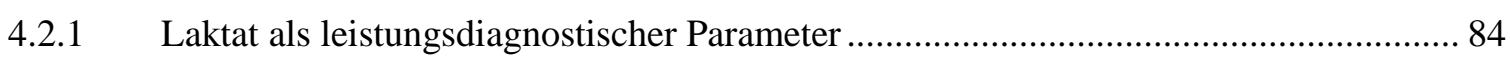

4.2.2 Vergleich sitzende und stehende Sportler - Wettkampf und Training.......................... 85 
4.2.3 Vergleich erfolgreiche und weniger erfolgreiche Sportler

4.2.4 Feldversuch versus unspezifische Messungen in der Vorbereitungsphase im Sommer 90

4.2.5 Vergleich Behindertensportler und gesunde Probandengruppe …............................... 91

4.2.6 Laktatkonzentrationskurven in der Nachbelastungsphase .............................................. 94

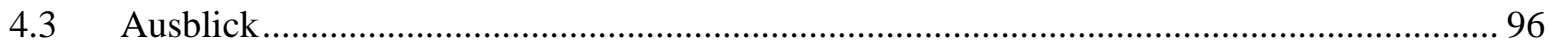

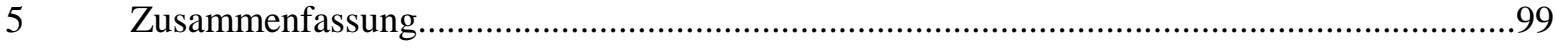

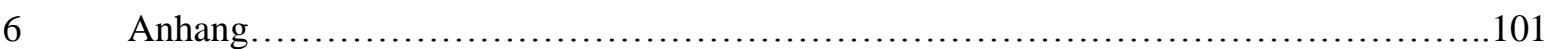

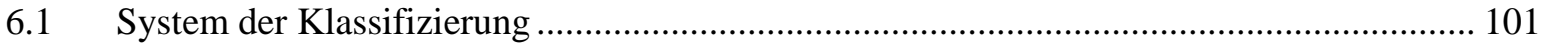

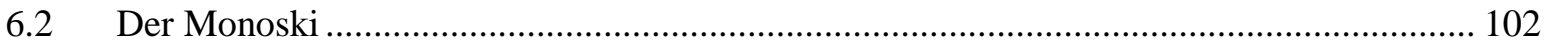

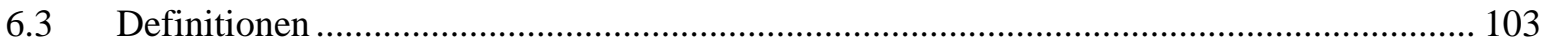

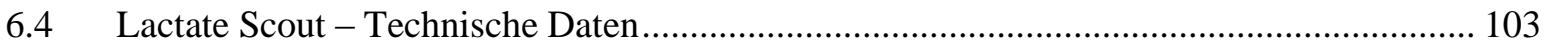

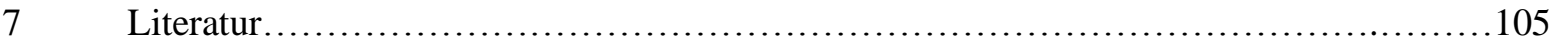


Abb.

Abbildung

Abs.

Absatz

Appl

Applied

ATP

Adenosintriphosphat

Aufl.

Auflage

bzw.

beziehungsweise

ca.

circa

DBS

Deutscher Behinderten- Sportverband e.V.

d.h.

das heißt

Disqu.

Disqualifikation

Diss.

Dissertation

DSB

Deutscher Sportbund

DSV

Deutscher Skiverband

Dtsch

Deutsche

et al.

et alii

etc.

et cetera

Eur

European

Hrsg.

Herausgeber

J

Journal

Jg.

Jahrgang

M.

$\max$.

musculus

MdE

maximal

min.

Minderung der Erwerbsfähigkeit

$\mathrm{Mm}$.

Minute/ Minuten

$\mathrm{mmol} / \mathrm{l}$

musculi

$\mathrm{NADH}$

Millimol pro Liter

Nikotinamidadenindinukleotid

od.

oder

Physiol

Physiology

R0

Laktatkonzentration im Blut in Ruhe vor Belastung (Rennen)

R1

Laktatkonzentration im Blut unmittelbar nach Belastungsende (Rennen) 
Laktatkonzentration im Blut 3 Minuten nach Belastungsende (Rennen)

R3

Laktatkonzentration im Blut 5 Minuten nach Belastungsende (Rennen)

R4

Laktatkonzentration im Blut 7 Minuten nach Belastungsende (Rennen)

RHC-KREK-System

Realistic Handicap Competition und Kreative Renn Ergebnis Kontrolle

S.

SoWi

Seite

Sportmed

Sozialwissenschaftliche

S0

Sportmedizin

S1

Laktatkonzentration im Blut in Ruhe vor Belastung (Sommer)

Laktatkonzentration im Blut unmittelbar nach Belastungsende (Sommer)

S2

Laktatkonzentration im Blut 3 Minuten nach Belastungsende (Sommer)

Laktatkonzentration im Blut 5 Minuten nach Belastungsende (Sommer)

Laktatkonzentration im Blut in Ruhe vor Belastung (Training)

Laktatkonzentration im Blut unmittelbar nach Belastungsende (Training)

Laktatkonzentration im Blut 3 Minuten nach Belastungsende (Training)

T3

Laktatkonzentration im Blut 5 Minuten nach Belastungsende (Training)

Tab. Tabelle

u.a.

unter anderem

v.

von

v.a.

vor allem

Vgl.

Vergleiche

WHO

World Health Organization

Z

Zeitschrift

z.B.

zum Beispiel 


\section{Abbildungsverzeichnis}

Abbildung 1: Kompartimente der Laktatproduktion, -verteilung und -elimination 11

Abbildung 2: Modell Leistungsstruktur Ski alpin - Riesenslalom 18

Abbildung 3: Modell Leistungsstruktur Ski alpin - Riesenslalom - Variable Faktoren 19

Abbildung 4: Rennen Jahr 1 - Alle Athleten 33

Abbildung 5: Rennen Jahr 2 - Alle Athleten 35

Abbildung 6: Rennen Jahr 3 (1. Messung) - Alle Athleten 36

Abbildung 7: Rennen Jahr 3 (2. Messung) - Alle Athleten 37

Abbildung 8: Athleten Rennen insgesamt - Sitzend und Stehend 38

Abbildung 9: Athleten Training insgesamt - Sitzend und Stehend 38

Abbildung 10: Athleten Sitzend Rennen gesamt 39

Abbildung 11: Rennen Jahr 1 - Sitzende Sportler $\quad 40$

Abbildung 12: Rennen Jahr 2 - Sitzende Sportler $\quad 41$

Abbildung 13: Rennen Jahr 3 (I.) - Sitzende Sportler 42

Abbildung 14: Rennen Jahr 3 (II.) - Sitzende Sportler 43

Abbildung 15: Athleten Stehend Rennen gesamt 50

Abbildung 16: Rennen Jahr 1 - Stehende Sportler

Abbildung 17: Rennen Jahr 2 - Stehende Sportler

Abbildung 18: Rennen Jahr 3 (I.) - Stehende Sportler 53

Abbildung 19 : Rennen Jahr 3 (II.) - Stehende Sportler $\quad 54$

Abbildung 20: Athleten Rennen gesamt - Sitzend und Stehend 64

Abbildung 21: Athleten Training gesamt - Sitzend und Stehend 65

Abbildung 22: Vgl. Rennen Sportler 1 und 14

$\begin{array}{ll}\text { Abbildung 23: Vgl. Rennen Sportler } 4 \text { und } 5 & 67\end{array}$

Abbildung 24: Vgl. Rennen Sportler 4 und $6 \quad 68$

$\begin{array}{ll}\text { Abbildung 25: Vgl. Rennen Sportler } 1 \text { und } 4 & 69\end{array}$

Abbildung 26: Vgl. erfolgreiche/ weniger erfolgreiche Sportler - Sitzend und Stehend 70 


\section{Tabellenverzeichnis}

Tabelle 1: Teilnehmende Sportler der jeweiligen Wettkampfjahre 25

Tabelle 2: Rennen Jahr 1 - Alle Athleten 33

Tabelle 3: Rennen Jahr 2 - Alle Athleten 34

Tabelle 4: Rennen Jahr 3 (1. Messung) - Alle Athleten 35

Tabelle 5: Rennen Jahr 3 (2. Messung) - Alle Athleten 36

Tabelle 6: Rennen Jahr 1 - Sitzende Sportler $\quad 40$

Tabelle 7: Rennen Jahr 2 - Sitzende Sportler $\quad 41$

Tabelle 8: Rennen Jahr 3 (I.) - Sitzende Sportler 41

Tabelle 9: Rennen Jahr 3 (II.) - Sitzende Sportler 42

Tabelle 10: Sportler 1- Rennen Jahre 1 bis 3

Tabelle 11: Sportler 1 - Training Jahr 1 und 2

Tabelle 12: Sportler 1 - Messergebnisse Sommer 44

Tabelle 13: Sportler 2 - Rennen Jahre 1 bis 3

Tabelle 14: Sportler 2 - Messergebnisse Sommer 45

Tabelle 15: Sportler 3 - Rennen Jahre 1 bis $3 \quad 46$

Tabelle 16: Sportler 3 - Training Jahr 1 und $2 \quad 46$

Tabelle 17: Sportler 3 - Messergebnisse Sommer $\quad 46$

Tabelle 18: Sportler 9 - Rennen Jahre 1 bis $3 \quad 47$

Tabelle 19: Sportler 9 - Messergebnisse Sommer $\quad 47$

Tabelle 20: Sportler 13 - Rennen Jahre 1 bis $3 \quad 48$

Tabelle 21: Sportler 13 - Training Jahr 1 und 2

Tabelle 22: Sportler 13 - Messergebnisse Sommer 48

Tabelle 23: Sportler 14 - Rennen Jahre 1 bis 3

Tabelle 24: Sportler 14 - Messergebnisse Sommer 49

Tabelle 25: Rennen Jahr 1 - Stehende Sportler 51

Tabelle 26: Rennen Jahr 2 - Stehende Sportler 51 
Tabelle 27: Rennen Jahr 3 (I.) - Stehende Sportler 52

Tabelle 28: Rennen Jahr 3 (II.) - Stehende Sportler 53

Tabelle 29: Sportler 4 - Rennen Jahre 1 bis 3

Tabelle 30: Sportler 4 - Training Jahr 1 und 2

Tabelle 31: Sportler 4 - Messergebnisse Sommer 55

Tabelle 32: Sportler 5 - Rennen Jahre 1 bis $3 \quad 56$

Tabelle 33: Sportler 5 - Training Jahr 1 und 2

Tabelle 34: Sportler 5 - Messergebnisse Sommer 57

Tabelle 35: Sportler 6 - Rennen Jahre 1 bis $3 \quad 57$

Tabelle 36: Sportler 6 - Training Jahr 1 und 2

Tabelle 37: Sportler 6 - Messergebnisse Sommer 58

Tabelle 38: Sportler 7 - Rennen Jahre 1 bis 3

Tabelle 39: Sportler 7 - Training Jahr 1 und 2

Tabelle 40: Sportler 7 - Messergebnisse Sommer

Tabelle 41: Sportler 8 - Rennen Jahre 1 bis $3 \quad 60$

Tabelle 42: Sportler 8 - Training Jahr 1 und $2 \quad 60$

Tabelle 43: Sportler 8 - Messergebnisse Sommer $\quad 61$

Tabelle 44: Sportler 10 - Rennen Jahre 1 bis $3 \quad 61$

Tabelle 45: Sportler 10 - Training Jahr 1 und $2 \quad 61$

Tabelle 46: Sportler 10 - Messergebnisse Sommer $\quad 62$

Tabelle 47: Sportler 11 - Rennen Jahre 1 bis 3

Tabelle 48: Sportler 11 - Training Jahr 1 und $2 \quad 62$

Tabelle 49: Sportler 11 - Messergebnisse Sommer 63

Tabelle 50: Sportler 12 - Rennen Jahre 1 bis $3 \quad 63$

Tabelle 51: Sportler 12 - Training Jahr 1 und 2 


\section{Einleitung}

\subsection{Problemstellung und Zielsetzung}

Im Mittelpunkt dieser Arbeit stehen leistungsdiagnostische Untersuchungen im Behindertensport Ski alpin. Skifahrer aus dem Leistungssportbereich werden trainingsbegleitend in Wettkampf, Training und in der Vorbereitungsphase im Sommer untersucht.

Im Behindertensport Ski alpin werden Breiten- und Leistungssport unterschieden. Der Leistungssportbereich erfährt eine zunehmende Professionalisierung, die jedoch im Vergleich zum Nichtversehrtensport weniger stark ausgeprägt ist.

Im Zuge dessen sind leistungsdiagnostische Untersuchungen mit dem Ziel einer Steuerung von Training und Wettkampf von großer Bedeutung. Unterschieden werden dabei sportartunspezifische, semispezifische und sportartspezifische Tests.

Erstere werden im Allgemeinen unter Laborbedingungen durchgeführt; so werden standardisierte und reproduzierbare Bedingungen erreicht.

Zur Durchführung sportartspezifischer Untersuchungen, also jener, in denen die Biomechanik der jeweiligen Sportart simuliert wird, eignen sich besonders zyklische Sportarten wie der Rad- oder der Rudersport. Derartige Messungen sind im Skisport unter Laborbedingungen nicht durchführbar, in Form eines Feldtests jedoch bereits in vielen Studien im Freizeit- und Leistungssportbereich im Nichtversehrtensport erfolgt.

Unter anderem wurden in der Arbeit von Temme (2001) sportartspezifische leistungsdiagnostische Messungen an Sportlern aus einer Weltcupmannschaft in verschiedenen Skidisziplinen in einem solchen Feldversuch am Hang vorgenommen.

Vergleichbare Untersuchungen existieren im Behindertenskisport bisher nicht.

Innerhalb eines Probandenkollektivs physisch beeinträchtigter Sportler findet sich ein breites Spektrum an Behinderungen. Dieser Besonderheit - also der Heterogenität der Gruppe gegenüber dem Nichtversehrtensport gilt die besondere Aufmerksamkeit. Die Bewegungsmöglichkeiten unterscheiden sich; dies wirkt sich wiederum auf die technische Ausführung und nicht zuletzt auf die Ergebnisse im Wettkampf aus. Durch eine Einteilung der Athleten im Wettkampf entsprechend ihrer physischen Beeinträchtigung in 
unterschiedliche Wettkampfklassen soll eine Vergleichbarkeit der Leistung und somit des Wettkampfergebnisses ermöglicht werden.

In dieser Arbeit werden nun sportartspezifische leistungsdiagnostische Untersuchungen anhand des Parameters Blutlaktat im Behindertensport Ski alpin ausgewertet. Sie ist auf Grund einer geringen Teilnehmerzahl und eines heterogenen Probandenkollektivs als Pilotstudie zu begreifen, die folgende Fragen thematisieren soll:

- Welche Besonderheiten gelten im Behindertensport Ski alpin?

- Welche Auswirkungen haben diese etwaigen Besonderheiten auf leistungsdiagnostische Untersuchungen?

- Sind leistungsdiagnostische Untersuchungen im Behindertenskisport im Rahmen eines Feldversuchs praktikabel?

- Welche Unterschiede sind von besonderer Bedeutung bei der Betrachtung sitzender und stehender Sportler?

- Bestehen Zusammenhänge zwischen Feldversuch im Winter und den unter Laborbedingungen im Sommer erhobenen Werten?

- Welche Bedeutung hat die Heterogenität des Probandenkollektivs hinsichtlich der Aussagekraft der Ergebnisse?

- Können die gewonnenen Ergebnisse im Rahmen einer Trainingssteuerung verwendet werden?

Der Behindertensport und Leistungsdiagnostik stehen in dieser Arbeit im Mittelpunkt. Es erfolgt zunächst eine Erläuterung der wichtigsten Begriffe und Hintergründe.

\subsection{Behindertensport}

\subsubsection{Definition Behinderung und Behindertensport}

Der Begriff Behinderung wird im Sozialgesetzbuch wie folgt definiert:

„Menschen sind behindert, wenn ihre körperliche Funktion, geistige Fähigkeit oder seelische Gesundheit mit hoher Wahrscheinlichkeit länger als sechs Monate von dem für das Lebensalter typischen Zustand abweichen und daher ihre Teilhabe am Leben in der Gesellschaft beeinträchtigt ist.“"(Sozialgesetzbuch IX, §2 Abs.1)

Im medizinischen Sinne ist eine Behinderung als eine „nicht nur vorübergehende, zu einer MdE (Minderung der Erwerbsfähigkeit) v. mindestens 10\% führende körperliche, geistige od. 
seelische Beeinträchtigung“ zu begreifen (Pschyrembel 1990, S.190), unabhängig von der Ursache.

Die WHO unternahm eine weitere Differenzierung in Schädigung (Impairment), Beeinträchtigung (Disability) und Behinderung (Handicap) (Vgl. Pschyrembel 2007, S. 221). Des Weiteren werden psychische und physische Behinderungen unterschieden.

„Unter Behindertensport werden alle Formen und Ausprägungen von Sport verstanden, die für Menschen mit Behinderung ausführbar sind.“ (Zimmer 2007, S. 451).

Der Deutsche Behindertensportbund unterteilt den Behindertensport in verschiedene Bereiche; in Rehabilitationssport, Breiten- und Leistungssport erfolgen unterschiedliche Zielsetzungen (Vgl. http://www.dbs-npc.de).

Im Rahmen der vorliegenden Arbeit werden Probanden aus dem Leistungssportbereich mit verschiedenen körperlichen Behinderungen untersucht; von besonderem Interesse ist der medizinische Aspekt des Behindertensports.

\subsubsection{Behindertensport Ski alpin}

Im Behindertensport Ski alpin bewegen sich Sportler mit verschiedenen körperlichen Behinderungen; eine große Unterscheidung ist zunächst - auf der technischen Ausführung basierend - zwischen sitzenden und stehenden Athleten vorzunehmen. In die Gruppe der sitzenden Sportler sind jene einzuordnen, die Querschnittlähmungen oder Beinamputationen aufweisen; die Behinderungen in der Gruppe Stehend reichen von Amputationen der Arme über Teilamputationen der Beine bis hin zu sehbehinderten Skifahrern.

Trotz dieses breiten Spektrums an körperlichen Behinderungen bestreiten die Athleten einen gemeinsamen Wettkampf. Um eine Vergleichbarkeit der Wettkampfergebnisse zu erzielen, werden sogenannte Wettkampfklassen formiert, in denen bestimmte Schädigungsmuster als Cluster zusammengefasst werden. Jede Gruppe erhält einen bestimmten Zeitfaktor, mit dem im Anschluss an den Wettkampf die echte Rennzeit (Absolutzeit) neu berechnet wird.

Im Zuge der Pseudonymisierung der Probanden in dieser Studie ist auch eine weitgehende Anonymisierung der Behinderungen notwendig. Dies geschieht im Folgenden über die Benennung der einzelnen Wettkampfklassen.

Zur Übersicht folgt eine genauere Darstellung des Klassifizierungssystems. 


\section{System der Klassifizierung}

Vor einem Wettkampf findet die Einordnung der jeweiligen Athleten in eine bestimmte Wettkampfklasse statt. Dem gehen umfangreiche Untersuchungen der Sportler durch anerkannte Physiotherapeuten und Ärzte voraus. Die Einordnung erfolgt anhand funktioneller Eigenschaften. Auch während des Rennens werden die Athleten durch Ärzte und Physiotherapeuten beobachtet, um etwaige Veränderungen der physischen Fähigkeiten registrieren zu können (Vgl. http:// www.skiteam-alpin.de 2008: Reglement: Die Klassifizierung).

Eine erste Unterscheidung der Sportler geschieht in die drei Kategorien

$$
\begin{aligned}
& \text { ○lind, } \\
& \text { ○ Stehend und } \\
& \text { ○ Sitzend. }
\end{aligned}
$$

Diese wiederum beinhalten mehrere Klassen (Vgl. http:// www.skiteam-alpin.de 2008: Reglement: Klassen. Klassen im Behindertensport Ski Alpin); eine ausführliche Darstellung des Klassifizierungssystems findet sich im Anhang.

Zur Erläuterung der Skitechnik innerhalb der Gruppen folgen einige Beispiele:

\section{a) Skifahrer der Kategorie Blind}

Sehbehinderte Skifahrer nehmen mit einem Guide an Skirennen teil. Dieser Guide fährt vor Ihnen und gibt ihnen über akustische Signale, beispielsweise „rechts, rechts, rechts“, die Kurvenrichtung an. Die Kategorie „Blind“ wird in drei Klassen unterteilt; jeder Athlet hat unabhängig vom Sehvermögen einen Guide.

\section{b) Stehende Skifahrer}

Das Spektrum der körperlichen Behinderungen in dieser Klasse ist groß.

Skifahrer, die armamputiert sind, fahren mit einem Stock oder ohne Stock. Jene, deren körperliche Behinderung an den unteren Extremitäten lokalisiert ist, nehmen unterstützend sogenannte Krückenski zur Hilfe. Dies sind kleine Ski, die der Stabilisierung dienen und je nach Situation hochgeklappt (zum Anschieben) oder flachgestellt (während des Fahrens) werden können (Vgl. Fendt 2002, S. 4). Sie werden über ein Zugseil betätigt; durch Zug können sie in ihrer Position verändert werden. Diese Krückenski finden ebenfalls bei Monoskifahrern Verwendung. 


\section{c) Sitzende Skifahrer - Monoskifahrer}

In die Kategorie Sitzend werden Skifahrer eingeordnet, die sich im Alltag mit einem Rollstuhl bewegen. Die Ursachen sind vielfältig - beispielsweise Querschnittlähmungen oder Amputationen der Beine. Diese Sportler bedienen sich eines sogenannten Monoskis; der Sportler sitzt in einer auf seine körperliche Behinderung abgestimmten Sitzschale, die auf einem Ski montiert ist (Bauweise des Monoskis siehe Anhang).

Zur Stabilisierung werden ebenfalls Krückenski eingesetzt, die an den Armen befestigt werden.

\section{Das Handicap-System}

Bei einem großen Spektrum an körperlichen Behinderungen von Sportlern bei einem gemeinsamen Wettkampf stellt die Bewertung der Leistung eine Herausforderung dar. Die erbrachte Leistung eines Skifahrers mit einer im oberen Thorakalmark lokalisierten Querschnittlähmung beispielsweise lässt sich schwer mit der eines Sportlers vergleichen, der eine Querschnittlähmung im unteren Lumbalbereich aufweist und demzufolge mehr aktive Muskelmasse zur Bewegungsausführung und Stabilisierung einsetzen kann.

Aus diesem Grund wurde das sogenannte „RHC-KREK-System (Realistic Handicap Competition und Kreative Renn Ergebnis Kontrolle) oder kurz Handicap System“

(http:// www. skiteam-alpin.de 2008: Reglement. Handycap-System. Das Handicap-System) entwickelt.

Dieses beinhaltet, dass verschiedenen Klassen innerhalb der drei Kategorien jeweils Zeitfaktoren zugeordnet werden. Im Anschluss an das Rennen wird die tatsächlich erzielte Laufzeit mit dem entsprechenden Faktor multipliziert. Die resultierende Zeit ist dann unmittelbar mit der anderer Athleten innerhalb derselben Kategorie vergleichbar (Vgl. http:// www. skiteam-alpin.de 2008: Reglement. Handycap-System. Das Handicap-System).

Aus diesen Ausführungen wird deutlich, dass das Probandenkollektiv im Rahmen der Untersuchungen und der Ergebnisse nicht zusammengefasst werden kann, eine Clusterbildung ist nicht möglich. Im Folgenden wird also eine Einzelbetrachtung vorgenommen.

Zentrales Thema der Arbeit sind leistungsdiagnostische Untersuchungen, im Folgenden wird Leistungsdiagnostik zunächst im Allgemeinen und anschließend im Zusammenhang mit dem Skisport und Behindertensport beleuchtet. 


\subsection{Leistungsdiagnostik}

\subsubsection{Leistungsdiagnostik im Allgemeinen}

Unter Leistungsdiagnostik versteht man „Lehre und Komplex von Verfahren der Leistungsdiagnose, d.h. der Erfassung und Beurteilung der sportlichen Leistungen und der aktuellen Leistungsfähigkeit - des erreichten Leistungsstandes - auf der Grundlage von Kennwerten, Kennlinien und Merkmalen des Leistungsvollzuges sowie von Kennwerten der wesentlichen personalen Leistungsvoraussetzungen“(Schnabel G 1997, S.49).

Im Rahmen einer Trainingsplanung und -steuerung werden in der Vorbereitungsphase auf einen Wettkampf leistungsdiagnostische Untersuchungen durchgeführt, die Rückschlüsse auf den Trainingszustand und die Leistungsfähigkeit des einzelnen Sportlers und zur weiteren Trainingsplanung erlauben sollen.

Je näher dabei der Bezug zur Leistungsstruktur einer Sportart ist, desto gezielter kann eine Trainingssteuerung vorgenommen werden (Vgl. Schnabel G et al. 2008, S. 450).

In der sportmedizinischen Leistungsdiagnostik werden „,sportartspezifische, semispezifische und unspezifische Prüfbedingungen“" unterschieden (Pansold 1983, S.9).

Sportartunspezifische Untersuchungen erfolgen im Labor und können beispielsweise mithilfe von Ergometern (Fahrrad, Ruderergometer, Laufband) als Stufentests durchgeführt werden; die Dauer der jeweiligen Belastungsstufen kann variiert werden. Verschiedene Parameter werden bestimmt: Maximale Belastung, Herzfrequenz, Laktatkonzentration, maximale Sauerstoffaufnahme sowie maximales Atemminutenvolumen (Vgl. Clasing 1994, S. 222).

Bei sitzenden Sportlern (Querschnittlähmungen) kommt das Handbike bzw. die Drehkurbelergometrie zum Einsatz; ,die Pedale werden durch Handgriffe ersetzt, die mit den Armen bewegt werden“ (de Marées 2002, S. 448).

Semispezifische Untersuchungen werden an Spezialergometern durchgeführt (Skilanglaufergometer). Sportartspezifische Untersuchungen hingegen geben den motorischen Stereotyp der Sportart wieder (Vgl. Bachl et al. 1994, S. 166). Sie können ebenfalls anhand von Laboruntersuchungen (Rad- / Rudersport) oder als Feldversuche unter Wettkampf- oder Trainingsbelastung vorgenommen werden. Sie dienen v.a. im Gegensatz zu Laboruntersuchungen der Beurteilung der sportartspezifischen Ausdauer (Vgl. Heck 1990, S. 100). 


\subsubsection{Laktat in der Leistungsdiagnostik}

„Die laktatorientierte Leistungsdiagnostik setzt die Konzentration des Laktats im Kapillarblut als Indikator für die Inanspruchnahme der anaeroben Glykolyse ein“ (Pessenhofer und Schwaberger 1994, S. 153).

\section{Energiebereitstellung unter Belastung}

Die Energiebereitstellung im Körper variiert in Abhängigkeit von der Belastungsdauer bei körperlicher Aktivität. Bei sehr kurzen Belastungen werden Phosphate zur Energiebereitstellung verwendet; bei länger andauernden (bis zu ca. zwei Minuten) wird die Energie über den Abbau von Glukose im Rahmen der Glykolyse zur Verfügung gestellt: Aus Glucose entsteht unter Gewinn von ATP Laktat.

In der vorletzten Stufe der Glykolyse entstehen Pyruvat und ATP. Das Pyruvat wird enzymatisch durch die Laktatdehydrogenase zu Laktat reduziert, dabei wird NADH oxidiert.

Diese Reaktion von Pyruvat zu Laktat ist sowohl unter aeroben als auch anaeroben Bedingungen möglich.

Die Energiebilanz unterscheidet sich in Abhängigkeit von aeroben und anaeroben Bedingungen: unter aeroben Bedingungen werden 36 bis 38 ATP, auf dem anaerobem Weg nur 2 ATP gewonnen. (Vgl. Löffler 2001, S. 107).

In der anaeroben Glykolyse ist Laktat (Laktatanion) das Endprodukt. Gleichzeitig fallen vermehrt Wasserstoffionen an, da NADH bei Sauerstoffmangel nicht oxidiert werden kann. Das Laktat hat keinen Einfluss auf den pH-Wert des Blutes, da es als Anion vorliegt und nur schlecht durch die Muskelmembran diffundiert. Eine Abnahme des Blut-pH während anaerober Arbeit ist auf eine ansteigende Wasserstoffionenkonzentration zurückzuführen (Vgl. Weicker 1994, S. 16).

Unter aeroben Bedingungen - also Belastungssituationen, in denen eine ausreichende Sauerstoffversorgung des Organismus gewährleistet ist - wird Laktat zu Pyruvat oxidiert, welches dann wiederum weiteren Abbauwegen zugeführt werden kann und Energie liefert.

Unter anaeroben Bedingungen kann diese Reaktion nicht erfolgen, so dass die Konzentration des Laktats im Blut ansteigt. Anschließend wird das Laktat in Abhängigkeit von der zu leistenden Arbeit eliminiert.

Bezüglich der Elimination des Laktats bestehen Unterschiede zwischen den verschiedenen Muskelfasertypen (Vgl. Weicker 1994, S. 17; Weineck 2007, S. 238). 
Wird kurzfristig starke körperliche Arbeit geleistet und werden damit anaerobe Energiebereitstellungswege in Anspruch genommen, steigt die Laktatkonzentration im Muskel an. Sie ist im Vergleich zum Blut ca. zweimal so hoch (Vgl. Kindermann und Keul, 1977b S. 61). Das Laktat wird anschließend durch die Zellmembran aus der Muskelzelle ins Blut transportiert, „nach kurzzeitigen erschöpfenden Belastungen bis etwa drei Minuten Dauer wird zwischen der 6. und 10. Minute der Erholungsphase ein annäherndes Äquilibrium zwischen Muskel- und Blutlactatkonzentration erreicht“ (Kindermann und Keul 1977b, S. 61).

Dauert eine körperliche Belastung länger als zwei Minuten, erfolgt die Energiebildung vorwiegend auf aerobem Weg. In Korrelation zur Veränderung der Laktatkonzentration im Blut bei anaerober Energiegewinnung verändern sich der $\mathrm{pH}-\mathrm{Wert}$ und der base exzess spiegelbildlich (Vgl. Kindermann und Keul 1977a, S. 81).

Das entstandene Laktat wird aus dem Blut in verschiedenen Organen dehydriert und Pyruvat letztlich in $\mathrm{CO}_{2}$ und Wasser umgewandelt, so dass der Laktatkonzentrationsspiegel im Blut wieder sinkt.

Durch leichte körperliche Betätigung nach Belastungsende kann der Abbau des Laktats beschleunigt werden (Vgl. Kindermann und Keul 1977b, S. 63).

\section{Laktatkinetik und Training}

Sportliches Training hat unterschiedliche Auswirkungen auf den Organismus: „neben morphologisch nachweisbaren Veränderungen ist eine Adaptation des Zellstoffwechsels zu erwarten“ (Keul 1969, S. 193). So konnte Keul nachweisen, „dass beim Training für die Ausdauerleistungen die oxydativen und beim Training für kurzfristige Muskelarbeit ... die glykolytischen Zellsysteme zunehmen“ (Keul 1969, S. 198).

Durch eine Zunahme der oxydativen Enzyme nimmt die Laktatkinetik einen anderen Verlauf; „der Laktatumsatz steigt mit Zunahme der Belastungsdauer und einer trainingsbedingten Zunahme der oxydativen Enzyme an“(Kindermann und Keul 1977b, S. 63).

In Untersuchungen an 400-m-Läufern konnten Kindermann und Keul feststellen, dass diese eine höhere Laktatazidose tolerieren können als untrainierte Sportler. Weiterhin spielt die Motivation der Spitzensportler eine entscheidende Rolle: Sie schütten vor und während der sportlichen Belastung in höherem Maße Katecholamine aus, die wiederum glykolytische Wirkung haben, die ,somit teilweise an der unterschiedlichen metabolischen Azidose beteiligt sein könnte“ (Kindermann und Keul 1977b, S. 82). Während der Untersuchungen wurde bei 
den beteiligten Sportlern bei den untrainierten Probanden eine höhere Laktatazidose beobachtet als bei den trainierten Sportlern. Es ist also anzunehmen, dass „das Verhalten des Säure-Basen-Haushalts im arteriellen Blut ... für die Bewertung des Trainingszustands oder der Leistungsfähigkeit unter normalen hämodynamischen Bedingungen von Bedeutung sein kann“ (Kindermann und Keul 1977b, S. 92).

Weiterhin „setzen Trainierte ihre Muskulatur ökonomischer ein, so dass die laktatproduzierenden weißen Fasern erst bei höheren Belastungen rekrutiert werden“" (Klinke und Silbernagl 2003, S.523).

Zusammengefasst bedeutet dies also, dass durch Training die Energiespeicher, die Mitochondriendichte und Enzymaktivitäten zunehmen und hormonelle Regulationsmechanismen optimiert werden (Vgl. Weineck 2007, S. 253.)

In Abhängigkeit von der Dauer körperlicher Belastung und den Hauptbewegungselementen verhält sich die Laktatkonzentration im Blut. Sie steigt bei Sportarten mit einem hohen Anteil an anaeroben Elementen. Bei Ausdauersportarten hingegen, bei denen vorwiegend auf aerobem Wege Energie bereitgestellt wird, ist die Blutlaktatkonzentration zumeist geringer.

Für Pansold sind die Laktatbildungsgeschwindigkeit sowie die Kapazität, also die Laktatmenge von Bedeutung. Die Systeme sind in unterschiedlicher Form zu trainieren. Durch Wiederholungen mit Belastungszeiten bis $\mathrm{zu} 20$ Sekunden sei ein immer wiederkehrender Reiz auf die Laktatbildungsgeschwindigkeit auszuüben. Wird hingegen die Belastung bei geringerer Wiederholungszahl verstärkt, wird ein Reiz auf die „glykolytische Durchsatzrate“" ausgeübt (Vgl. Pansold 1983, S.7).

Die Laktatkonzentration im Blut ist also abhängig von der Bildung des Laktats, seiner Verteilung im Kompartiment Blut und der Elimination.

\section{Anaerobe Ausdauer}

Bei sehr intensiven kurzzeitigen Belastungen, unter einer Dauer von zwei Minuten, steht also die anaerobe Energiegewinnung im Vordergrund.

Die anaerobe Ausdauer wird von Schnabel A et al. (1979, S. 503) auch als „Schnelligkeitsausdauer“ bezeichnet. 
Bezüglich der Leistung gibt es metabolische Faktoren, die diese begrenzen und deshalb berücksichtigt werden müssen:

„- die Energiefreisetzung aus den energiereichen Phosphaten (alactacide anaerobe Kapazität), die Energiefreisetzung aus der Glykolyse (lactacide anaerobe Kapazität) und die Widerstandsfähigkeit gegenüber der Lactatacidose (Acidosetoleranz)“(Schnabel A et al. 1979, S. 503).

Im Rahmen der laktatorientierten Leistungsdiagnostik wurden zahlreiche sogenannte Schwellenmodelle entwickelt, einen Überblick liefern Heck et al. (1985).

Mader entwickelte 1976 das Modell der „aerob-anaeroben Schwelle“; mit dem Begriff bezeichnete er den „Bereich des Übergangs zwischen der rein aeroben zur partiell anaeroben laktazid gedeckten muskulären Energiestoffwechselleistung“ (Heck et al. 1985, S. 22). Diesen Bereich siedelte er bei einer Laktatkonzentration von 4 mmol Laktat/l Blut an.

Im Folgenden entwickelten Kindermann, Keul und Simon abgewandelte Schwellenmodelle. Stegmann konstatierte 1981 die „individuell anaerobe Schwelle“, die er definierte „als den Zeitpunkt, an dem maximale Eliminationsrate und die Diffusionsrate des Laktats im Gleichgewicht stehen“ (Heck et al. 1985, S. 24).

Im physiologischen Sinne handelt es sich um eine Homöostase. Laktat wird in Abhängigkeit von der Belastungsform gebildet, im Kompartiment Blut verteilt und anschließend abgebaut. Diese Prozesse verlaufen überlappend; „zahlreiche metabolische und hormonelle Regelmechanismen beeinflussen die Produktion, den Release und die Elimination von Laktat" (Weicker 1994, S.11).

Ziel dieser Regulationsmechanismen ist, die Veränderungen des «inneren Milieus» so gering wie möglich zu halten (Vgl. Klinke und Silbernagl 2003, S. 535).

Im Allgemeinen kommt es bei körperlicher laktazider Belastung zu einem Anstieg des Laktats im Blut; diese ist zum einen auf eine Bildung des Laktats zum anderen jedoch auch auf eine Umverteilung der Durchblutung von Leber zu Skelettmuskel und dementsprechend einer „ungenutzten Lactatklärfunktion der Leber“ (Klinke und Silbernagl 2003, S.522) zurückzuführen.

Bei der Verteilung des Laktats im Blut und der gemessenen Konzentration sind verschiedene Mechanismen von Bedeutung: Enzymaktivitäten, Durchlässigkeit der Zellmembranen, Flüssigkeitsgehalt des Extrazellularraums und die Größe des Blutvolumens; die Laktatkonzentration ist also multifaktoriell bedingt (Vgl. Stegemann 1991, S.270). 
Ähnlich viele Faktoren beeinflussen die Elimination: der Trainingszustand des Herzens und die Regeneration des Laktats in der Leber beispielsweise (Vgl. ebenda 1991, S. 270); weiterhin Belastungs- und Überbelastungsreaktionen oder regenerative Maßnahmen (Vgl. Weicker 1994, S.18).

Die Laktatfreisetzung/-bildung variiert weiterhin in Abhängigkeit von statischer oder dynamischer Muskelarbeit.

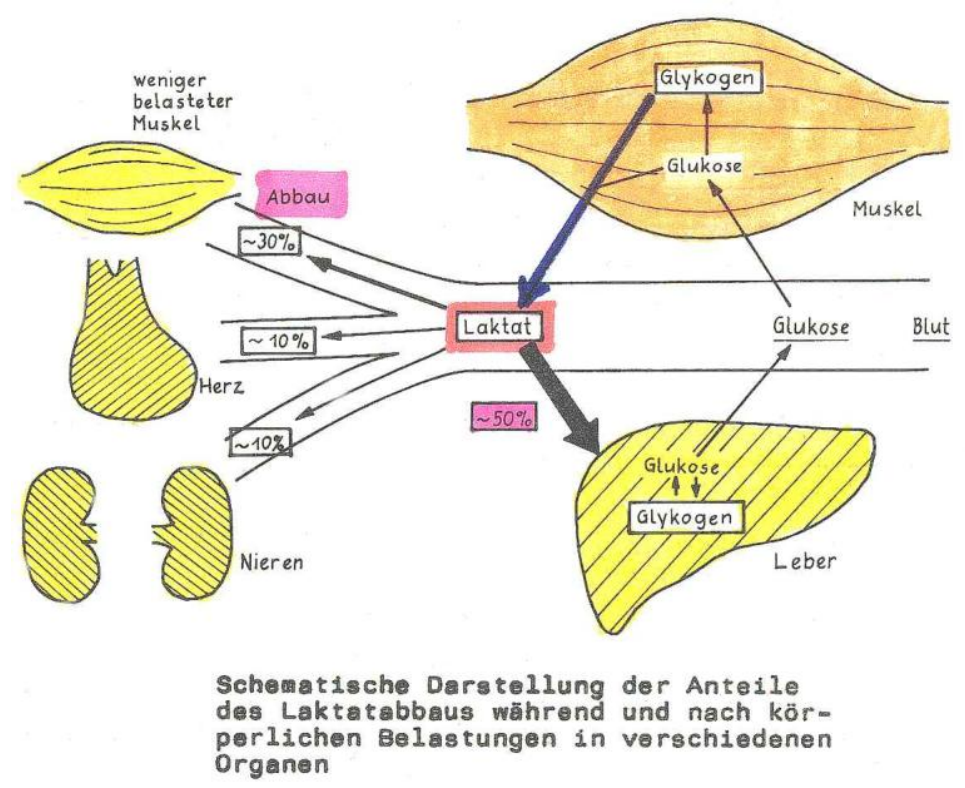

Abbildung 1: Kompartimente der Laktatproduktion, -verteilung und -elimination (Neumann und Gottschalk 1982, S. 17)

\section{Laktatkonzentrationskurven}

Entsprechend der Struktur einer Sportart ergeben sich verschiedene Verläufe der Laktatkonzentration während und nach einer Belastung. Eine Möglichkeit zur Beschreibung der Laktatkinetik bietet sich über die Darstellung anhand einer Laktatkonzentrationskurve.

Zunächst ist eine grundlegende Unterscheidung vorzunehmen in Laktatkonzentrationskurven, die die Kinetik während einer Belastung, und jene, die sie in der Nachbelastungsphase wiedergeben.

Pansold und Zinner konnten eine Laktatleistungskurve in der Sportart Schwimmen typisieren, die ebenso „für andere Sportarten unter sportartspezifischen, semispezifischen oder unspezifischen Testbedingungen" anwendbar ist (Pansold und Zinner 1994, S. 51).

Von Interesse bei der Interpretation der Laktatkonzentrationskurve sind vor allem das Anstiegsverhalten und die maximal gemessene Laktatkonzentration im Blut als „Ausdruck der anaerob-laktaziden Energiebereitstellung im Muskel“" (Pansold und Zinner 1994, S. 51). 
Training beeinflusst den Verlauf der Laktatkonzentrationskurve in unterschiedlicher Form:

Ein Training mit vorwiegend aerober Belastungskomponente kann zu einer Rechtsverschiebung der Kurve führen, während sich eine Verbesserung des glykolytischen Systems beispielsweise in Form höherer maximaler Laktatwerte ausdrücken würde (Vgl. Pansold 1983, S. 16). Zusammengefasst zeigt sich eine Verbesserung der Leistung in einer Rechtsverschiebung und Abflachung der Kurve sowie Änderung der maximalen Laktatwerte (Pansold und Zinner 1994, S. 52)

Die Analyse der Steigung der Laktatkonzentrationskurve zeigte in Untersuchungen, „daß die gleiche Steigung der Laktatkurve mit Verbesserung des Trainingszustands bereits bei einer geringeren Laktatkonzentration erreicht wird“"(Simon et al. 1981, S. 12).

Bei durchschnittlich trainierten Personen würde also die kritische Laktatzunahme im Gegensatz zu Ausdauertrainierten erst bei höheren Laktatwerten im Blut erreicht (Vgl. Simon et al., 1981, S. 12).

Über die Darstellung der Laktatkonzentration während einer Belastung soll eine Beurteilung des aktuellen Leistungsstands eines Sportlers ermöglicht werden (Vgl. Braumann et al. 1987, S. 37). Sie finden in der vorliegenden Studie vorwiegend Verwendung in den Sommermessungen der stehenden Athleten.

Laktatkonzentrationskurven, die die Kinetik in der Nachbelastungsphase wiedergeben, zielen hingegen v.a. auf eine Beurteilung der Regenerationsfähigkeit eines Sportlers ab.

Der maximale Wert der Laktatkonzentration nach Belastung ,ist unter Berücksichtigung von Diffusions- und Eliminationsprozessen ein direkter Indikator für das im Muskel gebildete Laktat“" (de Marées 2002, S. 479).

Insgesamt finden Laktatkonzentrationskurven in leistungsdiagnostischen Untersuchungen Anwendung, um Verlaufsbeobachtungen zur Beurteilung der Trainingseffektivität durchzuführen (Vgl. Braumann et al. 1987, S. 38).

Die Struktur der Sportart Ski alpin gibt bereits geeignete Messzeitpunkte vor. Untersuchungen in Ruhe sowie nach einem Wettkampf- oder Trainingsrennen sind einfach durchzuführen; Analysen während der Belastung sind kompliziert. Hinzu kommt, dass gerade im Riesenslalom eine gute Regenerationsfähigkeit auf Grund zweier unmittelbar aufeinanderfolgender Durchläufe von großer Bedeutung ist. Die Darstellung der Laktatkinetik in der Nachbelastungsphase ermöglicht eine Interpretation der Regenerationsfähigkeit; deshalb werden Laktatkonzentrationskurven nach Belastung konstruiert und analysiert. 
Koch und Raschka beschreiben verschiedene denkbare Modellierungen von Laktatkonzentrationskurven: So seien sowohl nicht-lineare Regressionsmodelle als auch Wachstumskurven geeignet, um eine konstante Belastung abzubilden (Vgl. Koch und Raschka 1999, S. 128). Durch Polynome lasse sich eine rein empirische Beschreibung des Kurvenverlaufs erreichen (Koch und Raschka 1999, S. 129). Grundsätzlich sollen Daten möglichst optimal an ein Modell angepasst werden; empirische Modelle erfüllen diese Voraussetzung. Mithilfe eines solchen Polynoms ist es auch möglich, Laktatkonzentrationen zu bestimmten Zeitpunkten zu berechnen. Eine weitere Möglichkeit zur Auswertung der Laktatkonzentrationskurve besteht in der Berechnung der Fläche unter der Kurve, um somit die Menge des insgesamt produzierten Laktats abschätzen zu können (Vgl. Koch und Raschka 1999, S. 128-130).

Das Probandenkollektiv dieser Studie weist ein großes Spektrum an körperlichen Behinderungen auf; die technische Ausführung differiert bereits zwischen sitzenden und stehenden Athleten: Auch bedienen sie sich unterschiedlicher Muskelmassen in unterschiedlicher Intensität.

Man könnte vermuten, ein ausschließlicher Vergleich der Laktatwerte nach Belastung eigne sich eher weniger zur Beurteilung der Regenerationsfähigkeit; über die Darstellung der Laktatkonzentration im Verlauf sollen Rückschlüsse ermöglicht werden. Weiterhin ist der Frage nachzugehen, ob und inwiefern die jeweilige Laktatkinetik eines Sportlers von der eines anderen abweicht.

Die physiologischen Prozesse sind vielschichtig und zeigen viele Abhängigkeiten. Ein starres Modell zur Interpretation dieser Prozesse - in diesem Fall der Laktatproduktion, -verteilung und des -abbaus - erscheint weniger geeignet.

Auf Grund dessen werden im Ergebnisteil Exponentialfunktionen und Polynome hinsichtlich ihrer Verwendbarkeit geprüft und gegenübergestellt. $\mathrm{Zu}$ untersuchen ist weiterhin, ob Berechnungen von Laktatkonzentrationen $\mathrm{zu}$ einem bestimmten Zeitpunkt $\mathrm{x}$ über diese Funktionen möglich wären.

\subsubsection{Besonderheiten der Leistungsdiagnostik im Behindertensport}

Besondere Berücksichtigung in dieser Studie sollte die Heterogenität des Kollektivs der ausgewählten Sportler finden.

Die Blutlaktatkonzentration variiert u.a. abhängig von der für eine Bewegungsausführung aktiv eingesetzten Muskulatur. Dies soll anhand eines Beispiels verdeutlicht werden: 
Sportler A sei querschnittgelähmt.

Sportler B sei blind.

Sportler A setzt zum Skifahren vor allem die obere Extremität und seine Rumpfmuskulatur ein, die Muskulatur der Beine kann nicht rekrutiert werden. Der Anteil der beanspruchten Muskulatur ist also verglichen mit Sportler B, der seine vollständige Muskulatur zum Skifahren einsetzen kann, deutlich erniedrigt.

Es ist anzunehmen, dass die Laktatkonzentration bei Sportler A wegen der geringeren Muskelmasse niedriger ist als bei Sportler B.

Diesen Zusammenhang konnten Aigner et al. bei Untersuchungen von Rollstuhlfahrern mit Querschnittlähmungen unterschiedlicher Lokalisation nachweisen: Die Laktatwerte waren umso höher, je niedriger der Querschnitt lokalisiert war und demzufolge mehr aktive Muskeln zur Bewegungsausführung eingesetzt werden konnten (Vgl. Aigner et al. 1990, S. 313 f.).

Wie bereits angesprochen ist die Laktatkonzentration im Blut von unterschiedlichen Faktoren abhängig. Sowohl die Laktatproduktion und -akkumulation als auch der Abbau des vorhandenen Laktats sind von der Muskelmasse abhängig, „der Anteil an laktatproduzierender Muskulatur, der Verteilungsräume, und die Eliminationsmöglichkeiten müssen berücksichtigt werden“ (Bachl et al. 1994, S. 173). Die Glykogenspeicher unterscheiden sich. Für die Studie bedeutet dies, dass auf Grund der verschiedenen körperlichen Behinderungen und der entsprechend unterschiedlich einsetzbaren Muskelmasse individuelle Laktatkonzentrationskurven beschrieben werden könnten. Daraus lässt sich die Notwendigkeit einer Einzelfallbetrachtung der erhobenen Daten ableiten.

Im Mittelpunkt des Interesses der Studie stehen also nicht die Absolutwerte der gemessenen Laktatkonzentration im Blut, sondern der Verlauf der Laktatkonzentration im Blut nach der Belastung, sie wird während der Erholungsphase bestimmt.

Als Vergleichsgrößen können vereinzelt Ergebnisse von standardisierten Ergometertests der jeweiligen Sportler aus der Vorbereitungsphase der entsprechenden Jahre dienen.

Die sportartspezifische Leistungsdiagnostik wird durch die Leistungsstruktur einer Sportart, also durch die einzelnen Elemente, beeinflusst. 
Über die Darstellung des Bewegungsprofils soll eine Leistungsstruktur für die Ski-alpinDisziplin Riesenslalom entwickelt werden, die ebenfalls für den Behindertensport Ski alpin Anwendung finden kann.

\subsection{Leistungsstruktur Riesenslalom}

Die Leistungsstruktur einer Sportart ist im Allgemeinen definiert als „innerer Aufbau der sportlichen Leistung aus bestimmten Faktoren (Elemente) und ihren Wechselbeziehungen (Kopplungen)“ (Schnabel G und Thieß 1987, S.113). Die sportliche Leistung setzt sich aus vielen Einflussgrößen zusammen, die untereinander in Beziehung stehen (Vgl. ebenda 1987, S.113).

Anhand der Struktur einer Sportart ist „das Aufstellen von Strukturmodellen künftiger Höchstleistung und eine dementsprechende Organisation und methodische Gestaltung des Trainings“" möglich (Ebenda 1987, S.114).

Aus der Definition der Leistungsstruktur ergibt sich weiterhin die Notwendigkeit einer Trainings- und Wettkampfstruktur.

Zur Entwicklung eines Modells der Leistungsstruktur einer Sportart müssen also Faktoren und Wechselbeziehungen definiert werden.

Im Skisport alpin werden vier Disziplinen unterschieden:

- Abfahrt

- Super-G

- Riesenslalom und

- Slalom.

Die Messungen im Rahmen der Studie wurden im Riesenslalom durchgeführt. Für diese Disziplin soll eine Leistungsstruktur konstruiert werden.

\section{Bewegungsprofil und Energiebereitstellung im Riesenslalom}

Im alpinen Skilauf dominieren Reaktionsbereitschaft, Schnelligkeit, Kraft und Muskelausdauer (Vgl. Keul et al. 1975, S. 53).

Die Muskelarbeit besteht aus statischen und dynamischen Elementen, diese sind in Abhängigkeit von der Disziplin unterschiedlich gewichtet. Während eines Rennens leistet ein 
Skifahrer v.a. „muskuläre Haltearbeit (vorrangig der Beinmuskulatur), die eine hohe Muskelausdauer erfordert“" (Kindermann und Keul 1977b, S. 73).

Im Riesenslalom finden sich durch häufige Richtungswechsel - auf Grund von mehr Kurven als in der Abfahrt und kleineren Kurvenradien - viele dynamische Elemente. Es werden zwei Renndurchläufe durch die Athleten absolviert; eine gute Grundlagenausdauer und Regenerationsfähigkeit sind also bedeutend.

Weiterhin sind eine gute neuromuskuläre Koordination und Reaktionsfähigkeit Voraussetzung.

Lochner et al. beschreiben, dass im Skirennlauf v.a. „Technik, Rennerfahrung und emotionelle Anpassung“ von Bedeutung sind (Lochner et al. 1983, S. 120).

Bein- und Rumpfmuskulatur kommen bei der Bewegungsausführung besonders zum Tragen (Vgl. Jonath und Krempel 1981, Abb. S. 403).

In vielen Untersuchungen, die die kardiopulmonale Belastung und die Energiebereitstellung im Skisport thematisierten, konnte gezeigt werden, dass beim alpinen Skilauf kurzfristig hohe Herzfrequenzen als Folge hyperadrenerger Reaktionen zu verzeichnen sind (Vgl. Keul et al. 1975, S. 50). Insgesamt gilt, „daß dynamische Bewegungsabläufe mit relativ vielen und großräumigen Teilkörper-Verlagerungen unter energetischen Gesichtspunkten das HerzKreislauf-System vergleichsweise stark beanspruchen“ (Mester und de Marées 1981, S. 86). Herzfrequenz und Blutdruck (systolischer ebenso wie diastolischer) steigen an (Vgl. Hollmann 1982, S. 190).

Eine weitere Ursache für die hohe Herzfrequenz ist die psychische Belastung.

Kindermann und Keul sind der Ansicht, Skiabfahrtslauf werde wegen der hohen psychischen Beanspruchung zu den Stressbelastungen gezählt. Auf Grund dessen seien Laktatanstieg und pH-Abfall nur zum Teil auf eine muskuläre Beanspruchung zurückzuführen (Vgl. Kindermann und Keul 1977a, S. 83). Die kardiopulmonale Belastung für den Organismus ist auf Grund der vorherrschenden Haltearbeit und nur kurzfristiger Muskelanspannungen jedoch gering (Vgl. Keul et al. 1975, S. 50), so dass sie keine Adaptationsvorgänge beispielsweise hinsichtlich der Herzgröße oder der aeroben Kapazität nach sich zieht (Vgl. Keul et al. 1975, S. 50; Keul et al. 1982, S. 126).

Die Muskelausdauer beinhaltet die aerobe und die anaerobe Ausdauer; auch diese sind je nach Disziplin unterschiedlich gewichtet. Insgesamt ist die Belastung kurz und hoch; dadurch ,ist 
der Organismus auf eine maximale Sauerstoffnutzung angewiesen und bevorzugt daher den Kohlenhydrateabbau“ (Keul et al. 1975, S. 56).

Die Dauer eines Renndurchlaufs beläuft sich jeweils auf eine Zeit von etwa einer Minute, so dass von einer vorwiegend anaeroben Energiebereitstellung auszugehen ist; dies kann wiederum starke Laktatanstiege zur Folge haben (Vgl. Lochner et al. 1983, S.118 f.).

Eine gute aerobe Ausdauer ist wichtig, jedoch ist gerade im Leistungssportbereich eine „allgemeine anaerobe Ausdauer (Schnelligkeitsausdauer)...leistungsbestimmend“ (Jonath und Krempel 1981, S. 398).

Lochner et al. zählen den alpinen Rennlauf im Hinblick auf den Energieumsatz zu jenen Wettkampfdisziplinen mit hohem Krafteinsatz (Vgl. Lochner et al. 1983, S. 118).

Nicht zuletzt wird durch die Katecholaminausschüttung (durch erhöhten Sympathikotonus) die Glykolyse unterstützt, da Schlüsselenzyme dieses Stoffwechselweges aktiviert werden (Vgl. Kindermann und Keul, 1977b, S. 74). Dies hat einen zunächst starken Anstieg der Laktatspiegel zur Folge. Keul et al. konnten in Untersuchungen nachweisen, dass die Laktatkonzentrationen nach einem Rennen ,20 bis 30 Minuten nach der Abfahrt wieder auf 3 bis $4 \mu \mathrm{Mol} / \mathrm{ml}$ abgesunken sind“" (Keul et al. 1975, S. 52).

Die anaerobe Glykolyse ist also v.a. im Riesenslalom wesentlich (Vgl. Lochner et al. 1983, S. 119), denn gerade in der Steuerphase des Schwunges wird die Durchblutung der Oberschenkelmuskulatur z.T. unterbrochen, da „,im Bereich der Knie- und HüftgelenkStreckmuskulatur durch anhaltende isometrische Kontraktion der Zentrifugalkraft entgegengewirkt werden muß“ (Mester und de Marées 1981, S. 86).

Keul et al. nehmen an, dass beim alpinen Skiläufer die Leistungsgrenzen durch die neuromuskuläre Koordination und die Energieumsetzungen im Muskel mit nachfolgender entsprechender Kontraktionsarbeit begrenzt würden (Vgl. Keul et al. 1982, S. 124).

Zuletzt sind die äußeren Faktoren nicht $\mathrm{zu}$ vernachlässigen: Wind, Schnee und Schneebeschaffenheit, Temperatur, Gelände und Geländebeschaffenheit sowie das Material (Vgl. dazu auch Fecht 1980, S. 85-95).

In Anlehnung an das Modell zum Anforderungsprofil Ski alpin von Jonath und Krempel (1981, S. 400) wurde aus den zuvor genannten Elementen eine Leistungsstruktur für die Disziplin Riesenslalom konstruiert. 


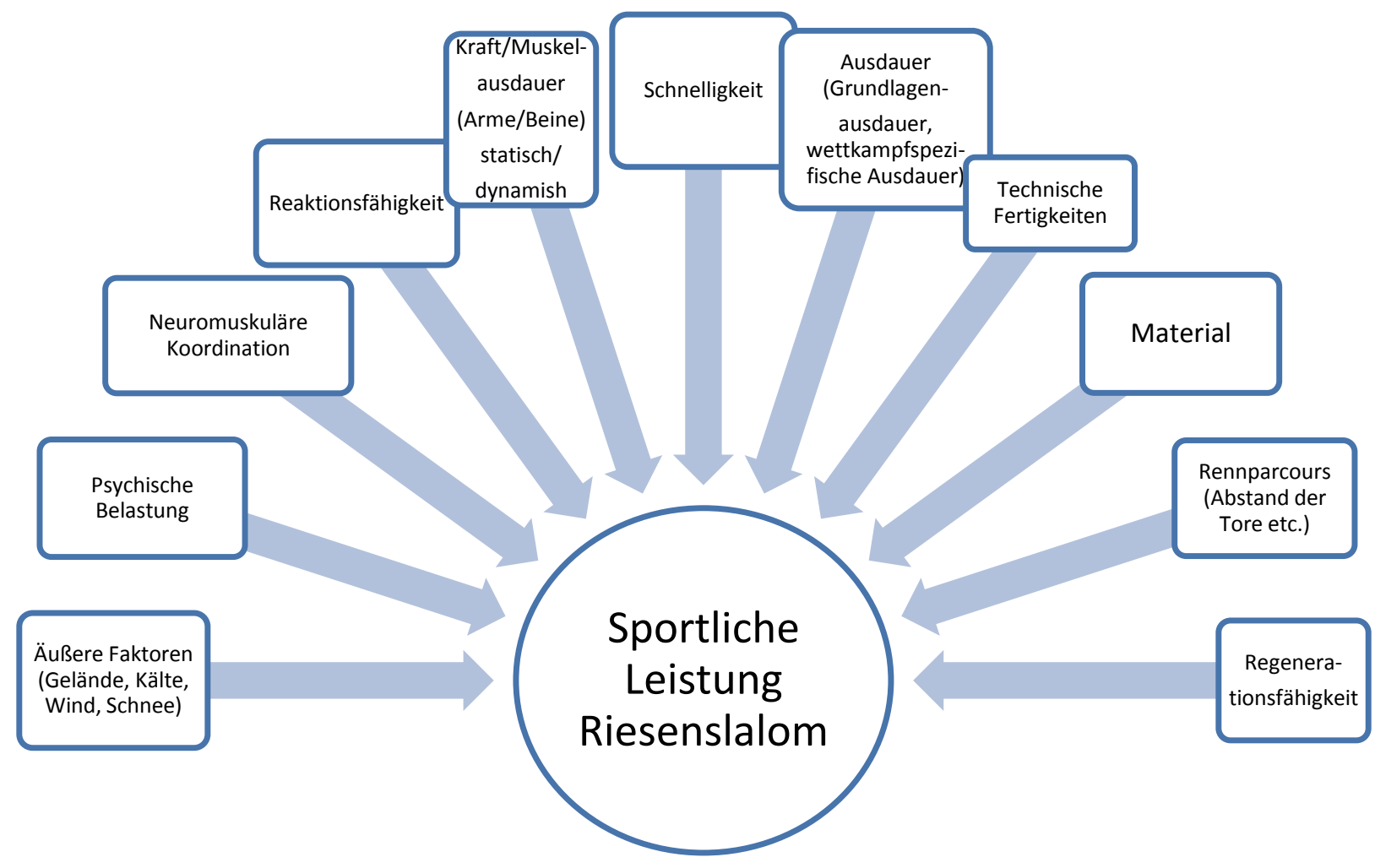

Abbildung 2: Modell Leistungsstruktur Ski alpin - Riesenslalom

Im Hinblick auf die Zusammensetzung der Probandengruppe müssen einige Elemente besonders bewertet werden.

Im Behindertensport kommen Besonderheiten gegenüber dem Nichtversehrtensport hinzu, die es zu berücksichtigen gilt und die als variable Elemente in der folgenden Abbildung farblich unterlegt sind. 


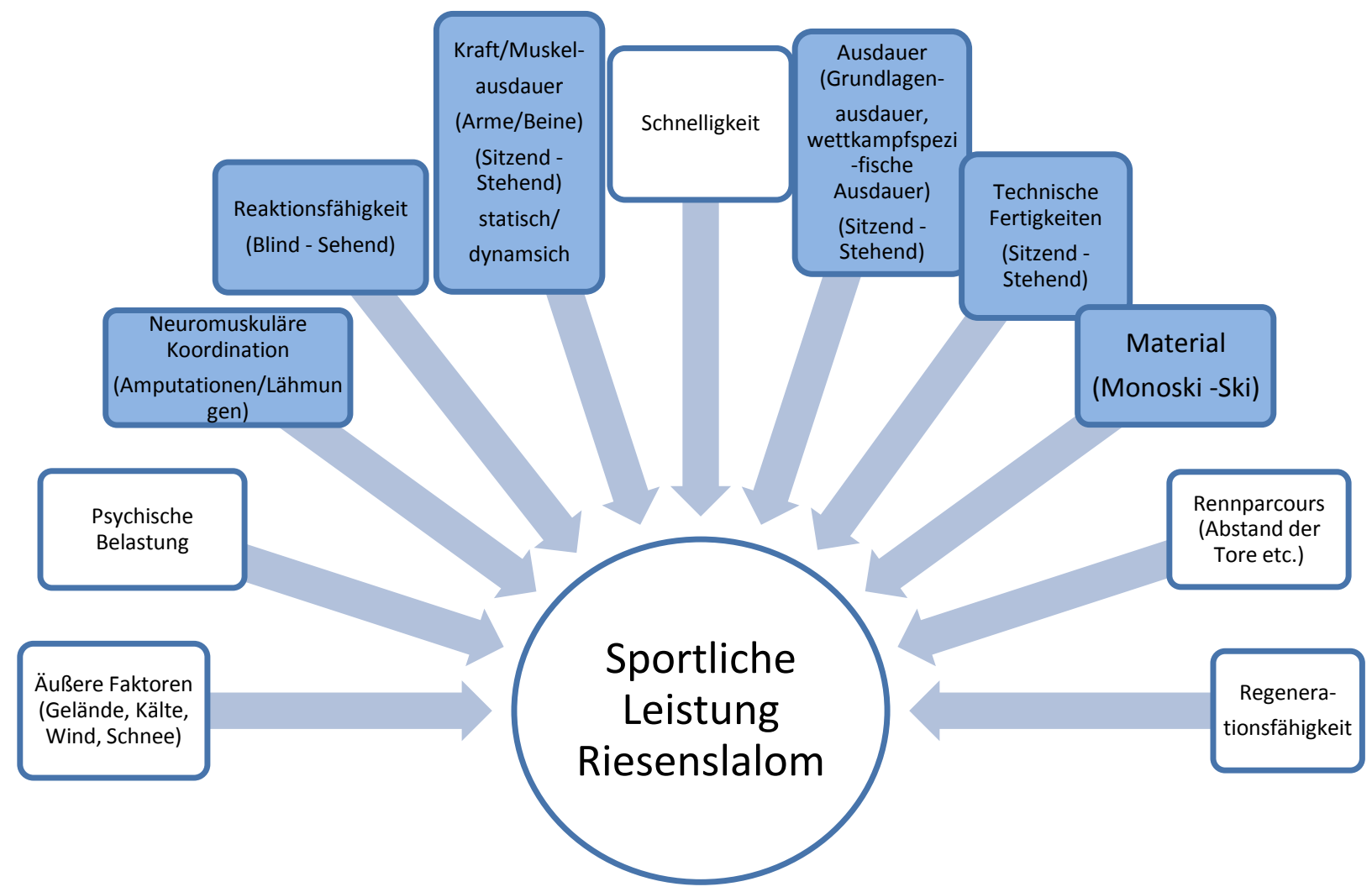

Abbildung 3: Modell Leistungsstruktur Ski alpin - Riesenslalom - Variable Faktoren

Die beanspruchte Muskulatur ist bei körperlich behinderten Skifahrern unterschiedlich verteilt: Stehende Skifahrer können je nach Ausprägung ihrer Behinderung die Muskeln im Bein- und Rumpfbereich zur Bewegungsausführung einsetzen. Sitzende Skifahrer hingegen müssen diese Bewegungen durch die Arme und Schultern sowie den Rumpf ersetzen.

Doch auch im Hinblick auf andere Faktoren bestehen Unterschiede zwischen gesunden und behinderten Sportlern sowie innerhalb der Gruppe der behinderten Athleten.

Vergleicht man beispielsweise einen blinden und einen sehenden Skifahrer, so wird deutlich, dass sich die Reaktionsfähigkeit unterscheidet; auch die neuromuskuläre Koordination oder Muskelausdauer variieren zwischen körperlich behinderten und nichtversehrten Sportlern. Insgesamt differieren auch Ausdauer, technische Fertigkeiten und das Material.

Sportmedizinische Leistungsdiagnostik hegt den Anspruch, dass es „Ziel jedes Leistungsprüfverfahrens sein [sollte], die aktuelle Belastung möglichst physikalisch bzw. motorisch-strukturell zu definieren“ (Bachl et al. 1994, S.167). Dies macht eine Definition der Leistungsstruktur Riesenslalom notwendig. 
An der Variabilität der Elemente bei Anwendung auf die Gesamtprobandengruppe wird deutlich, dass auf Grund verschiedener Schädigungsmuster und dementsprechend unterschiedlicher Bewegungsmöglichkeiten eine Einzelfallbetrachtung auch der leistungsdiagnostischen Ergebnisse von Nöten ist.

\subsection{Leistungsdiagnostische Untersuchungen im Skisport alpin}

In der Literatur sind einige leistungsdiagnostische Untersuchungen von verschieden strukturierten Probandengruppen im Feldversuch am Skihang zu finden. Diese erfolgten alle im Nichtversehrtensport. Verschiedene Parameter wurden bestimmt, u.a. auch die Laktatkonzentration im Blut vor und nach einer Belastung; die Höhe der Konzentrationen ist dabei sehr unterschiedlich beschrieben.

Festzuhalten ist zunächst, dass die Laktatkonzentration abhängig vom Können eines Skifahrers und der Belastungsform ist.

Eher niedrige Laktatwerte werden im Freizeitsportbereich beschrieben:

Berghold et al. (1984) untersuchten die Laktatkonzentration im Blut nach Belastung bei geübten Tourenskiläufern zunächst nach dem Aufstieg und anschließend nach der Abfahrt. Dieser Gruppe gegenüber stellten sie weitere Probanden, die ausschließlich eine Abfahrt absolvierten. Insgesamt wurde jeweils ein Nachbelastungswert bestimmt. Der mittlere Maximalwert betrug 2,5 $\pm 0,9 \mathrm{mmol} / \mathrm{l}$ bei der erstgenannten Gruppe und 2,6 $\pm 1,1 \mathrm{mmol} / \mathrm{l}$ in der Abfahrtsgruppe.

Mester und de Marées (1981) führten Laktatmessungen während verschiedener Untersuchungseinheiten mit fortgeschrittenen und guten Skifahrern durch; die Maximalwerte nach Belastung lagen höher als bei Berghold et al., sie stiegen bis zu einer Konzentration von $6,95 \mathrm{mmol} / \mathrm{l}$ Blut (Mittelwert 4,55 \pm 1,11 mmol/l) bei der Übungsform Abfahrt an. In der Untersuchungsreihe war v.a. der Anstieg der Laktatkonzentration von Interesse.

Röder (2001) nahm vergleichende Untersuchungen u.a. der Laktatkonzentration bei Freizeit-, Seniorenskifahrern und aktiven Skilehrern vor. Sie konnte nachweisen, dass die Gruppe der Skilehrer als geübteste Probandengruppe mit einer mittleren Laktatkonzentration von 2,66 \pm $0,83 \mathrm{mmol} / \mathrm{l}$ niedrigere Werte als alle anderen, vor allem als die Seniorenskifahrer $(3,65 \pm$ 
$1,11 \mathrm{mmol} / \mathrm{l}$ ) aufwiesen. Vergleichend schloss sich eine Versuchsreihe auf einem Abfahrtssimulator an.

Röder bietet weiterhin einen weitreichenden Überblick über andere empirische Studien im Skisport und ihre Ergebnisse (Vgl. Röder 2001, S. 13). So werden auch zwei Studien angeführt, in denen deutlich höhere Laktatkonzentrationen ermittelt wurden: Raas/ Hörtnagel (1984) und Zintl/ Gebauer (1985).

Insgesamt konnten höhere Laktatkonzentrationen in Untersuchungen nachgewiesen werden, deren Probanden aus dem Leistungssportbereich rekrutiert wurden bzw. bei denen die Belastung höher war - sei es durch schwierige Geländeverhältnisse oder längere Belastungszeiten. Dies waren v.a. Belastungsformen, die vergleichsweise viele dynamische Bewegungselemente enthielten.

In den zuvor erwähnten Studien von Raas und Hörtnagel (1984) sowie Zintl und Gebauer (1985) wurden Untersuchungen an Leistungssportlern während eines Riesenslaloms vorgenommen, als Probanden fungierten Kaderskiläufer. Sie ermittelten Laktatkonzentrationen in der Nachbelastungsphase von 15,2 $\pm 2,1 \mathrm{mmol} / \mathrm{l}$ (Raas und Hörtnagel) bzw. 10-11 mmol/l (Zintl und Gebauer) (Vgl. Röder 2001, S. 13).

Lochner et al. (1983) führten Laktatmessungen an Skilehrern nach einem Riesenslalomrennen unter Wettkampfbedingungen durch; maximal wurde eine Laktatkonzentration von 9,9 mmol/l bestimmt. Auch bei dieser Studie war der Anstieg der Laktatwerte durch die Belastung von vorrangigem Interesse, es wurde ein Wert nach drei Minuten in der Nachbelastungsphase bestimmt.

Saibene et al. (1985) und Veicsteinas et al. (1984) untersuchten Skifahrer auf nationalem Niveau. Saibene et al. simulierten ein Riesenslalomrennen, als Probanden fungierten Athleten aus dem Leistungssportbereich; nach Beenden der Belastung wurde in der gesamten Gruppe ein Maximalwert von 8,3 mmol/l erreicht. Veicsteinas et al. untersuchten ebenfalls Skifahrer aus dem Leistungssportbereich. Die Laktatkonzentrationen wurden im Rahmen eines Riesenslaloms aus venösem Blut aus der Kubitalvene in Ruhe und fünf Minuten nach Belastungsende bestimmt; der mittlere Maximalwert betrug 12,4 $\pm 1,9 \mathrm{mmol} / \mathrm{l}$.

Mayr (2008) begleitete Freeskier (Leistungssportler auf internationalem Niveau) während einiger Wettkämpfe und bestimmte verschiedene metabolische Parameter im 
Belastungsverlauf. Laktat wurde vor und nach dem Rennen ermittelt. Auch er führte lediglich die Messung eines Nachbelastungswerts durch (mittlerer Maximalwert 12,4 \pm 2,6 mmol/l, Maximalwert eines Sportlers $16 \mathrm{mmol} / \mathrm{l})$.

Hintzy et al. (2009) untersuchten den Anstieg der Laktatkonzentration nach einem Riesenslalomrennen an jugendlichen Rennläufern; die gemessenen Laktatwerte lagen mit max. 5,7 mmol/l deutlich unter den Ergebnissen der oben angeführten Studien. Von Bedeutung ist hierbei, dass Jugendliche eine andere Zusammensetzung der Skelettmuskulatur aufweisen und sich somit Laktatproduktion, -verteilung und -elimination unterscheiden (Vgl. Hintzy et al. 2009, S. 500).

Temme (2001) bestimmte in ihren Untersuchungen von Leistungssportlern im Feldversuch u.a. im Riesenslalom neben anderen Parametern die Laktatkonzentration im Blut vor und bis 30 Minuten nach Belastung. Die Mittelwerte der einzelnen Athleten - unterteilt in Frauen und Männer - wurden ermittelt. Bei den Frauen betrug der Maximalwert 13,8 mmol Laktat/l Blut (Mittelwerte nach Belastung ca. 9,6 bis $12 \mathrm{mmol} / \mathrm{l}$ ), bei den Männern wurden Werte über 20 mmol/l gemessen (Mittelwerte nach Belastung ca. 15 -17 mmol/l). 
Folgende Hypothesen sollen im Rahmen dieser Arbeit überprüft werden:

\subsection{Hypothesen}

- Laktat ist als leistungsdiagnostischer Parameter für sportartspezifische Untersuchungen im Riesenslalom geeignet.

- Sitzende und stehende Athleten zeigen Unterschiede in der Höhe der Laktatkonzentration und eine andere Laktatkinetik nach Belastung.

- Erfolgreiche und weniger erfolgreiche Sportler zeigen Unterschiede in der Höhe der Laktatkonzentration und eine andere Laktatkinetik nach Belastung.

- Es bestehen Unterschiede hinsichtlich der Laktatkonzentration bei behinderten und gesunden Sportlern.

- Trotz Heterogenität der Gruppe und Einzelfallanalysen lassen sich aus den leistungsdiagnostischen Untersuchungen Schlussfolgerungen hinsichtlich einer Trainingsplanung ableiten.

- Leistungsdiagnostische Untersuchungen im Behindertensport Ski alpin sind im Feldversuch praktikabel und aussagekräftig hinsichtlich einer Trainingssteuerung. 


\section{Material und Methode}

\subsection{Durchführung}

In der Studie Trainingsbegleitende Leistungsdiagnostik im Behindertensport Ski alpin wurden leistungsdiagnostische Untersuchungen anhand des Parameters Laktat im Vollblut vorgenommen. Als Probanden fungierten Sportler, die sich im Leistungssportbereich bewegen. Sie weisen verschiedene körperliche Behinderungen auf; die Struktur der Gruppe ist also heterogen. Sie wurden vom Mannschaftsarzt für die Studie vorgeschlagen; die Geschlechterverteilung ist gemischt.

Der Mannschaftsarzt hat im Rahmen seiner Betreuung in drei aufeinander folgenden Jahren während vier Wettkämpfen und zwei dazugehörigen Trainingseinheiten kapilläre Blutentnahmen aus dem Ohrläppchen zur Bestimmung der Laktatkonzentration im Blut der Sportler durchgeführt. Das Laktat wurde also aus dem Vollblut bestimmt. Dies ist insofern von Bedeutung, als die Laktatkonzentration im Plasma auf Grund der niedrigeren Konzentration in Erythrozyten höher ist (Vgl. de Marées 2002, S. 473). Ergänzend liegen Ergebnisse von sportartunspezifischen Messungen aus der Vorbereitungsphase der vorangegangenen Sommer vor.

Diese Blutentnahmen wurden außerhalb der Studie im Rahmen der normalen Betreuung der Sportler vorgenommen. Die Leistungsdiagnostik ist Bestandteil des Trainings und dient der Optimierung der Trainingsinhalte und der Gestaltung der Trainingsintervalle. Es handelt sich um eine Routinemaßnahme, die unabhängig von der Studie stattfindet.

Im Rennen wurde bei jedem Sportler die Blutlaktatkonzentration in Ruhe vor Belastung (R0) bestimmt. Weitere Messungen erfolgten wegen der Struktur des Riesenslaloms nach der Belastung: 1.) unmittelbar nach Belastungsende (Wettkampfende) (R1), 2.) drei Minuten nach Belastungsende (R2), 3.) fünf Minuten nach Belastungsende (R3) und zum Teil sieben Minuten nach Belastungsende (R4).

Während des Trainings wurden Proben zu den Zeitpunkten T0 (in Ruhe), T1 (unmittelbar nach Belastungsende), T2 (drei Minuten nach Belastungsende) und T3 (fünf Minuten nach Belastungsende) entnommen. Im Sommer hat die Probengewinnung zum einen während der Belastung, zum anderen in der Nachbelastungsphase (S0, S1, S2, S3) entsprechend den Zeitpunkten aus Rennen und Training stattgefunden.

Anhand der Werte soll der Verlauf der Laktatkonzentration im Blut in der Phase nach Belastung beschrieben werden. 
Der folgenden Tabelle ist zu entnehmen, wann die einzelnen Sportler an den Messungen teilgenommen haben. Insgesamt liegen vier Messreihen im Rahmen von Wettkämpfen, zwei während der vorbereitenden Trainingseinheiten sowie drei aus den Messungen aus der Vorbereitungsphase im Sommer vor.

\begin{tabular}{|c|c|c|c|c|c|c|c|c|c|c|c|c|c|}
\hline Sportler & 1 & 2 & 3 & 4 & 5 & 6 & 7 & 8 & 9 & 10 & 11 & 12 & 13 \\
\hline Teilnahme Rennen Jahr 1 & $x$ & & $x$ & $x$ & $x$ & $x$ & $x$ & $x$ & $x$ & $x$ & & $x$ & \\
\hline Teilnahme Rennen Jahr 2 & $x$ & & $x$ & $x$ & $x$ & $x$ & $x$ & $x$ & & $x$ & $x$ & & $x$ \\
\hline Teilnahme Rennen Jahr 3 (I.) & $x$ & $x$ & & $x$ & $x$ & $x$ & $x$ & & & & & & \\
\hline Teilnahme Rennen Jahr 3 (II.) & $x$ & $x$ & & $x$ & $x$ & $x$ & $x$ & & & & & & \\
\hline Teilnahme Training Jahr 1 & $x$ & & & $x$ & $x$ & & $x$ & & & & $x$ & $x$ & \\
\hline Teilnahme Training Jahr 2 & $x$ & & $x$ & $x$ & $x$ & $x$ & $x$ & $x$ & & $\mathrm{x}$ & $x$ & & $x$ \\
\hline Teilnahme Sommermessung Jahr 1 & $x$ & $x$ & $x$ & $x$ & $x$ & $x$ & $x$ & & $x$ & $x$ & & & $x$ \\
\hline Teilnahme Sommermessung Jahr 2 & $x$ & & $x$ & $x$ & $x$ & & & $x$ & & & $x$ & & $x$ \\
\hline Teilnahme Sommermessung Jahr 3 & $\mathrm{x}$ & & $x$ & & & $x$ & & & & & & & \\
\hline
\end{tabular}

Die Erhebung der Messwerte im Sommer - während der Vorbereitungsphase - ist bei den sitzenden Sportlern in Form einer leistungsdiagnostischen Untersuchung mit einem Handbike erfolgt, das wiederum auf einem Laufband gesteuert wurde. Über die Einstellung der Geschwindigkeit des Laufbandes werden Belastungsstufen festgelegt, die reproduzierbar sind. In der Gruppe der stehenden Sportler ist eine leistungsdiagnostische Untersuchung mit Hilfe eines Fahrradergometers durchgeführt worden; die Belastungsstufen wurden zwischen 25 und 50 Watt gesteigert. In regelmäßigen Abständen (je Belastungsstufe) erfolgten Messungen von Herzfrequenz und Laktatkonzentration.

Als Messgerät während Sommer- und Wintermessungen fungierte der „Lactate Scout“ der Firma SensLab (technische Daten siehe Anhang). Die Bestimmung der Laktatkonzentration im Blut erfolgte anhand von kapillärem Blut aus dem Ohrläppchen.

In der Bedienungsanleitung des Gerätes ist ein Temperaturbereich von $+5^{\circ} \mathrm{C}$ bis $+45^{\circ} \mathrm{C}$ angegeben. Um im Feldversuch optimale Bedingungen zu schaffen, wurde das Gerät unter der Kleidung möglichst nah am Körper getragen.

Die Datenerhebung beginnt mit Trainingswerten, die einen Tag vor dem Rennen gewonnen wurden. Am Renntag erfolgte eine Ruhemessung (R0) bei Zimmertemperatur vor 
Besichtigung der Rennstrecke. Anschließend besichtigten die Athleten den Rennparcours und kehrten abhängig von ihrer Startzeit noch einmal in den Raum zurück. Erst unmittelbar vor dem Start verließen die Sportler den Raum. Als Skidisziplin der Wettkampfmessungen wurde der Riesenslalom gewählt.

Die Disziplinen Slalom und Super-G wurden erst nach dem Riesenslalom bestritten, so dass dies die erste Renndisziplin war, und die Athleten ausgeruht waren.

Die Erhebung der Daten nach Belastung (R1 bis R4) erfolgte bei Außentemperaturen.

Auf Grund der zuvor angesprochenen Problematik einer erschwerten Vergleichbarkeit der Leistungen der Athleten wird bei der Auswertung der Ergebnisse eine Unterteilung in die Gruppen Stehend und Sitzend in Anlehnung an die Einteilung im Wettkampf vorgenommen; Vergleiche erfolgen zunächst zwischen den Sportlern innerhalb derselben Gruppe.

Die Vielfalt der körperlichen Behinderungen der an der Studie teilnehmenden Sportler ist groß, in Abhängigkeit des jeweiligen Schädigungsmusters variiert das Bewegungsausmaß eines jeden Einzelnen. Es erfolgt eine Darstellung der einzelnen Schädigungsmuster und Bewegungsmöglichkeiten anhand der Darstellung der Wettkampfklassen.

\subsection{Vorstellung der einzelnen Athleten und Behinderungen}

\subsubsection{Sportler 1 (Klasse LW10/2: Monoskiläufer (wenig Rumpfmuskulatur))}

Bei Sportler 1 liegt eine komplette Querschnittlähmung im Bereich des Thorakalmarks vor. Die Funktionen von Schulter- und Armmuskulatur sind erhalten; Außenrotation und Abduktion des Arms sind möglich, weiterhin Innenrotation, Adduktion, Ante- und Retroversion des Armes nach medial. Der Einsatz der Krückenski ist gewährleistet.

Die autochthone sowie die sekundäre Thoraxmuskulatur sind ebenfalls in ihrer Funktion erhalten, der Thorax kann somit verspannt werden.

Durch die Läsion des Rückenmarks im Bereich des Thorakalmarks ist die Bauchmuskulatur im unteren Anteil von dieser Schädigung betroffen. Durch die segmentale Innervation ist eine Beurteilung der verbleibenden Funktion der Muskulatur erschwert. Im Falle des M. rectus abdominis bedeutet die Schädigung beispielsweise, dass die Anteile unterhalb des betroffenen Nervensegments nicht kontrahiert werden können; eine Rumpfbeugung im Sinne einer Annäherung von Os pubis und Sternum ist nicht möglich. Weiterhin betroffen sind die Mm. obliquus abdominis internus et externus, der M. transversus abdominis sowie die autochthone 
und sekundäre Rückenmuskulatur unterhalb der Läsion. Die unteren Anteile der Rückenmuskulatur und die Hüfte können nicht aktiv zur Bewegungsausführung eingesetzt werden.

\subsubsection{Sportler 2 (Klasse LW11: Monoskiläufer (mit Rumpfmuskulatur))}

Sportler 2 hat traumatisch bedingt eine komplette Querschnittlähmung in Höhe des unteren Thorakalmarks.

Die bei Sportler 1 beschriebenen Bewegungen der Arme, Schultern und des oberen Rumpfes sind Sportler 2 ebenfalls uneingeschränkt möglich, somit auch der Einsatz der Krückenski.

Das Verletzungsmuster ermöglicht eine fast vollständige Innervation der Bauchmuskeln (Ausnahme M. obliquus internus und Anteile des M. transversus abdominis), so dass Rotationsbewegungen und Annäherungsbewegungen von Thorax und Becken ausgeführt werden können. Durch den Einsatz der autochthonen und sekundären Rückenmuskulatur wird eine Streckung des Rückens und Verspannung des Rumpfes und der Wirbelsäule ermöglicht, auf Grund der Läsionshöhe im Bereich der unteren Brustwirbelsäule kann jedoch keine Dorsalflexion des Rückens erfolgen. Ebenfalls wird die Hüfte nicht muskulär stabilisiert.

\subsubsection{Sportler 3 (Klasse LW12/2: Monoskiläufer (Doppeloberschenkelamputiert))}

Sportler 3 ist doppeloberschenkelamputiert. Die oberen Extremitäten und der Rumpf sind zur Bewegungsausführung einsetzbar. Schulter-, Arm-, Bauch- und Rückenmuskulatur sind in ihrer Funktion erhalten, die Wirbelsäule kann vollständig bewegt werden (Ventral- und Dorsalflexion, Lateralflexion, Rotation). Auch die Hüfte ist in ihrer Funktion nicht eingeschränkt, so dass sowohl Flexionsbewegungen als auch Sitzstabilität gewährleistet sind.

\subsubsection{Sportler 4 (Klasse LW 5/7- 2: Behinderung an beiden oberen Extremitäten)}

Sportler 4 hat Amputationen unterschiedlicher Lokalisation an beiden Armen. Die Funktion der Beinmuskulatur ist komplett erhalten, so dass jegliche Bewegungen der Beine durchgeführt werden können.

Die Schulter- und Armmuskulatur des einen Armes und die Brust- und Rückenmuskulatur sind ebenfalls funktionell nicht beeinträchtigt, alle Bewegungen im Bereich des Rumpfes sind möglich. Die Stabilisierung des Rumpfes ist von besonderer Bedeutung, da die Stabilität durch verschiedene Amputationshöhen an den Armen gestört ist. Über den Einsatz eines Armes kann bezüglich des Gleichgewichts beim Skifahren ebenso wie durch eine vermehrte muskuläre Rumpfverspannung sowie die Beine korrigierend eingegriffen werden. 
Rotationsbewegungen des Rumpfes sind zu vermeiden, da sich eine Rotationsbewegung des Oberkörpers auf die untere Körperhälfte übertragen kann. Dies hat zur Folge, dass ein Schwung nicht mehr exakt gefahren werden kann.

Der Athlet fährt mit einem Stock, der speziell befestigt wird.

\subsubsection{Sportler 5 (Klasse LW2 : Einseitig-Oberschenkelamputiert - Krückenskiläufer)}

Sportler 5 ist einseitig in Höhe des Oberschenkels beinamputiert. Die Muskulatur von Armen und Rumpf ist funktionell nicht eingeschränkt, dies gilt ebenfalls für das gesunde Bein und die Hüfte.

Mit Hilfe von Unterarmgehstützen bewegt sich Sportler 5 im Alltag, beim Skifahren fährt er einbeinig mit Unterstützung durch spezielle Kurzski (Krückenski), die vergleichbar mit denen eines Monoskifahrers sind und an beiden Unterarmen befestigt werden, um durch die Arme den Stand und die Abfahrt zu stabilisieren.

\subsubsection{Sportler 6 (Klasse LW 6/8: Behinderung an einer oberen Extremität - Einstockfahrer)}

Sportler 6 hat eine einseitige Behinderung im Bereich der oberen Extremitäten, die einen nur bedingten Einsatz des Armes erlaubt. Tätigkeiten der Arme werden durch die gesunde Seite übernommen. Die Funktion der Brust- und Rücken- sowie der Beinmuskulatur ist ohne Einschränkung erhalten.

Der Athlet fährt ebenfalls wie Sportler 4 mit einem Stock, der jedoch keiner speziellen Befestigung bedarf, da der gesunde Arm voll funktionsfähig ist. Der beeinträchtigte Arm wird während des Skifahrens am Rumpf fixiert, um Rotationsbewegungen des Rumpfes zu reduzieren und Verletzungen der Arme durch Torstangen zu vermeiden. Stabilität wird über eine vermehrte Verspannung der Rumpfmuskulatur erreicht.

\subsubsection{Sportler 7 ( Klasse LW 6/8: Behinderung an einer oberen Extremität)}

Bei Sportler 7 ist ebenfalls die Funktion eines Armes auf Grund einer Behinderung beeinträchtigt. Tätigkeiten der Arme werden durch den gesunden Arm übernommen; Bauch-, Rücken-, Hüft- und Beinmuskulatur sind vollständig einsatzfähig.

Auch bei diesem Schädigungsmuster besteht beim Skifahren die Gefahr der Rotation des Rumpfes und der Verletzung des Armes durch Torstangen, jedoch besitzt der Athlet Kontrolle über den Oberarm und Ellenbogen, so dass eine Befestigung des Armes während des Trainings oder Wettkampfs nicht notwendig ist. Auch bei ihm erfolgt eine Stabilisierung des Rumpfes über eine vermehrte Verspannung der Brust-, Bauch- und Rückenmuskulatur. 
Sportler 7 fährt ebenfalls mit einem Stock.

\subsubsection{Sportler 8 ( Klasse B2: Sehbehindert wenig Sehrest)}

Sportler 8 ist sehbehindert; er besitzt eine geringe Restsehkraft, die sich auf das Umrisserkennen von Gegenständen beschränkt.

All seine Muskeln besitzen ihre vollständige Funktion. Er bewegt sich auf Grund des stark eingeschränkten Sehvermögens vorsichtiger. Beim Skifahren zeigt er eine verhaltenere, defensivere Fahrweise.

\subsubsection{Sportler 9 (Klasse LW11: Monoskiläufer (mit Rumpfmuskulatur))}

Sportler 9 hat eine traumatisch verursachte tiefe Querschnittlähmung. Die Funktion der Muskeln unterhalb der Läsion des Rückenmarks ist vollständig erloschen.

Das Bewegungsausmaß von Sportler 9 ist vergleichbar mit dem von Sportler 2. Die Arm- und Rumpfmuskulatur ist vollständig intakt, so dass er die Arme beim Monoskifahren unterstützend einsetzen kann. Im Rumpf ist sowohl die autochthone als auch die sekundäre Muskulatur in ihrer Funktion erhalten, so dass die Stabilisierung und die Bewegungen des Rumpfes möglich sind. Ventral- und Dorsalflexion sowie eine Lateralflexion bis zur Läsionshöhe sind uneingeschränkt durchführbar. Entscheidend ist beispielsweise im Vergleich zu Sportler 3, dass keine aktive Bewegung der Hüfte möglich ist. Die Hüftmuskulatur kann nicht nerval angesteuert werden. Ein Kippen des Beckens in der Sitzschale des Monoskis erfolgt also über eine Verspannung des Rumpfes und eine Gewichtsverlagerung des Rumpfes unter Zuhilfenahme der Arme, die stützend eingesetzt werden.

\subsubsection{Sportler 10 (Klasse LW 6/8-2: Behinderung an einer oberen Extremität - Einstockfahrer)}

Sportler 10 ist einseitig unterhalb des Ellenbogens armamputiert. Die Beinmuskulatur ist vollständig einsetzbar. Er fährt mit einem Stock. Die Funktion der Schulter ist erhalten. Eine Stabilisierung des Armes während der Abfahrt ist wichtig, damit das Verletzungsrisiko an den Torstangen minimiert wird. Eine weitere Schwierigkeit besteht darin, dass durch die Verletzung im Bereich des Oberkörpers ein Ungleichgewicht entsteht und daraus resultierend eine Rotationsbewegung, eine konsequente Stabilisierung des Rumpfes ist also bedeutend. 


\subsubsection{Sportler 11 (Klasse LW 2: Einseitig- Oberschenkelamputiert - Krückenskiläufer)}

Sportler 11 ist stehender Skifahrer, ein Bein ist in Höhe des Oberschenkels amputiert. Die Funktionalität des anderen Beines ist vollständig erhalten. Er setzt zur Stabilisierung der Fahrt zwei kurze Krückenski ein. Da er in leicht gebeugter Oberkörperhaltung fährt, sind die Muskeln des Rumpfes von besonderer Bedeutung.

\subsubsection{Sportler 12 (Klasse LW 6/8-2: Behinderung an einer oberen Extremität - Einstockfahrer)}

Sportler 12 ist einseitig unterarmamputiert, so dass er stehend Ski fährt. Der Arm ist eingeschränkt einsetzbar, die Rumpfmuskultur ist in ihrer vollen Funktion erhalten. Sportler 12 bedient sich eines Stockes auf der gesunden Seite. Wie bereits bei Sportler 6 beschrieben, liegt die Schwierigkeit in der Stabilisierung des beeinträchtigten Armes, so dass keine Verletzungen durch die Torstangen verursacht werden.

\subsubsection{Sportler 13 (Klasse LW 12-1: Monoskiläufer (niedrige inkomplette Querschnittlähmung))}

Sportler 13 hat eine tief lokalisierte inkomplette Querschnittlähmung. Er kann unter Zuhilfenahme von Unterarmgehstützen laufen, beim Skifahren jedoch bedient er sich eines Monoskis. Die Funktion der Rücken- und Bauchmuskultur ist zu einem Großteil erhalten. Er kann zur Stabilisierung des Rumpfes einen größeren Anteil seiner Rumpfmuskulatur einsetzen als beispielsweise Sportler 1. Die Hüftbewegung erfolgt mit Unterstützung durch die Rumpfmuskulatur.

\subsubsection{Sportler 14 (Klasse LW 11: Monoskiläufer (mit Rumpfmuskulatur))}

Sportler 14 ist Monoskifahrer, der seine Rumpfmuskulatur einsetzen kann. Dies hat eine gewisse Sitzstabilität zur Folge.

Die Muskulatur der Arme, des Schultergürtels und des Rumpfes ist vollständig rekrutierbar. Sportler 14 kann stabil sitzen und während des Skifahrens den Rumpf muskulär verspannen. Weiterhin kann er über die erhaltene Funktion der Rücken- und Bauchmuskultur ein Kippen des Rumpfes während der Kurvenfahrt erreichen. Die Hüftmuskulatur kann nicht aktiv eingesetzt werden. Die Arme dienen mithilfe der Krückenski der Stabilisierung. 


\subsection{Biometrie}

Die vorliegende Studie ist eine Pilotstudie und thematisiert trainingsbegleitende sportartspezifische leistungsdiagnostische Untersuchungen im Behindertensport Ski alpin.

Die Zusammensetzung der Probandengruppe ist heterogen; das Kollektiv beinhaltet insgesamt vierzehn Athleten, die - zunächst in die Gruppen Sitzend und Stehend unterteilt unterschiedliche physische Behinderungen aufweisen.

Die Auswertung der gewonnenen Daten erfolgt in Form einer Einzelfallbetrachtung, die Statistik ist zunächst deskriptiv. Mittelwerte und Standardabweichungen werden bestimmt.

Laktatkonzentrationskurven beschreiben die Laktatkinetik über die Zeit nach Belastung; als Modelle zur Darstellung werden Polynome dritter Ordnung und Exponentialfunktionen diskutiert.

Zum Vergleich der Gruppen untereinander und mit einer Vergleichsgruppe werden Varianzanalysen (ANOVA) durchgeführt. Die Auswertung der Daten geschieht mit dem Statistikprogramm SAS.

Ergebnisse mit einem Signifikanzwert $\mathrm{p}<0,05$ gelten als signifikant, $\mathrm{p}<0,1$ wird als Trend begriffen.

Die Auswertung gestaltet sich wegen der geringen Probandenzahl und der Heterogenität als schwierig; sie dient v.a. dem Ziel, Trends zu beschreiben. 


\section{Ergebnisse}

In der vorliegenden Studie wurden leistungsdiagnostische Untersuchungen anhand des Parameters Blutlaktat vorgenommen.

Die Laktatkonzentration im Blut wurde zu verschiedenen Zeitpunkten gemessen:

im Rennen/ Training/ Sommer

- R0/T0/ S0 Laktatkonzentration im Blut in Ruhe vor Belastung,

- R1/T1/S1 Laktatkonzentration im Blut unmittelbar nach Belastungsende,

- R2/T2/S2 Laktatkonzentration im Blut 3 Minuten nach Belastungsende,

- R3/T3/ S3 Laktatkonzentration im Blut 5 Minuten nach Belastungsende,

- R4 Laktatkonzentration im Blut 7 Minuten nach Belastungsende.

Insgesamt liegen vier Datenreihen aus dem Wettkampf (Rennen) vor: Jahr 1, Jahr 2 und Jahr 3 I und II; zwei Datenreihen aus dem Training: Jahr 1 und 2; drei Datenreihen aus der Vorbereitungsphase im Sommer: Jahr 1, 2 und 3.

Im Folgenden sind zunächst die Messergebnisse der teilnehmenden Athleten im Wettkampf nach den jeweiligen Jahren aufgeführt. 


\subsection{Ergebnisse aller Athleten - Wettkampf}

\subsubsection{Jahr 1}

Tabelle 2: Rennen Jahr 1 - Alle Athleten

\begin{tabular}{|c|c|c|c|c|c|}
\hline \multirow[b]{2}{*}{ Sportler Jahr 1} & \multicolumn{5}{|l|}{ Laktat (mmol/l) } \\
\hline & $\begin{array}{l}\text { Laktat Rennen } \\
\text { Ruhe (R0) }\end{array}$ & sofort (R1) & $\begin{array}{l}\text { nach } 3 \text { min. } \\
(\mathrm{R} 2)\end{array}$ & $\begin{array}{l}\text { nach } 5 \text { min. } \\
\text { (R3) }\end{array}$ & $\begin{array}{l}\text { nach } 7 \text { min. } \\
(\mathrm{R} 4)\end{array}$ \\
\hline Sportler 1 & 2 & 3,7 & 3,2 & 3,6 & 3,4 \\
\hline Sportler 3 & 1,5 & 2,7 & 3,1 & 3 & \\
\hline Sportler 4 & 3 & 6,1 & 7,2 & 7,3 & 7,7 \\
\hline Sportler 5 & 2,6 & 5,2 & 8,2 & 10,7 & 7,5 \\
\hline Sportler 6 & 1,8 & 3,8 & 3,7 & 3,8 & \\
\hline Sportler 7 & 1,6 & 4,3 & 4,5 & 4,4 & 3,5 \\
\hline Sportler 8 & 2,1 & 6,8 & 5,1 & 6,1 & 6,4 \\
\hline Sportler 9 & 2,1 & 3,2 & 4 & 3,5 & 2,8 \\
\hline Sportler 10 & 2,7 & 4,5 & 6,9 & 7 & 5,3 \\
\hline Sportler 12 & 2,5 & 3,8 & 6 & 6,8 & 5,7 \\
\hline
\end{tabular}

Im ersten Jahr haben insgesamt zehn Sportler an den Messungen teilgenommen. Im Gesamtüberblick zeigt sich, dass die Laktatkonzentrationen aller Sportler nach dem Ruhewert R0 ansteigen. Die Zeitpunkte des Erreichens der maximalen Laktatkonzentration im Blut der einzelnen Athleten variieren zwischen R1 und R4, auch variiert die Höhe der Maximalkonzentration des Laktats im Blut deutlich.

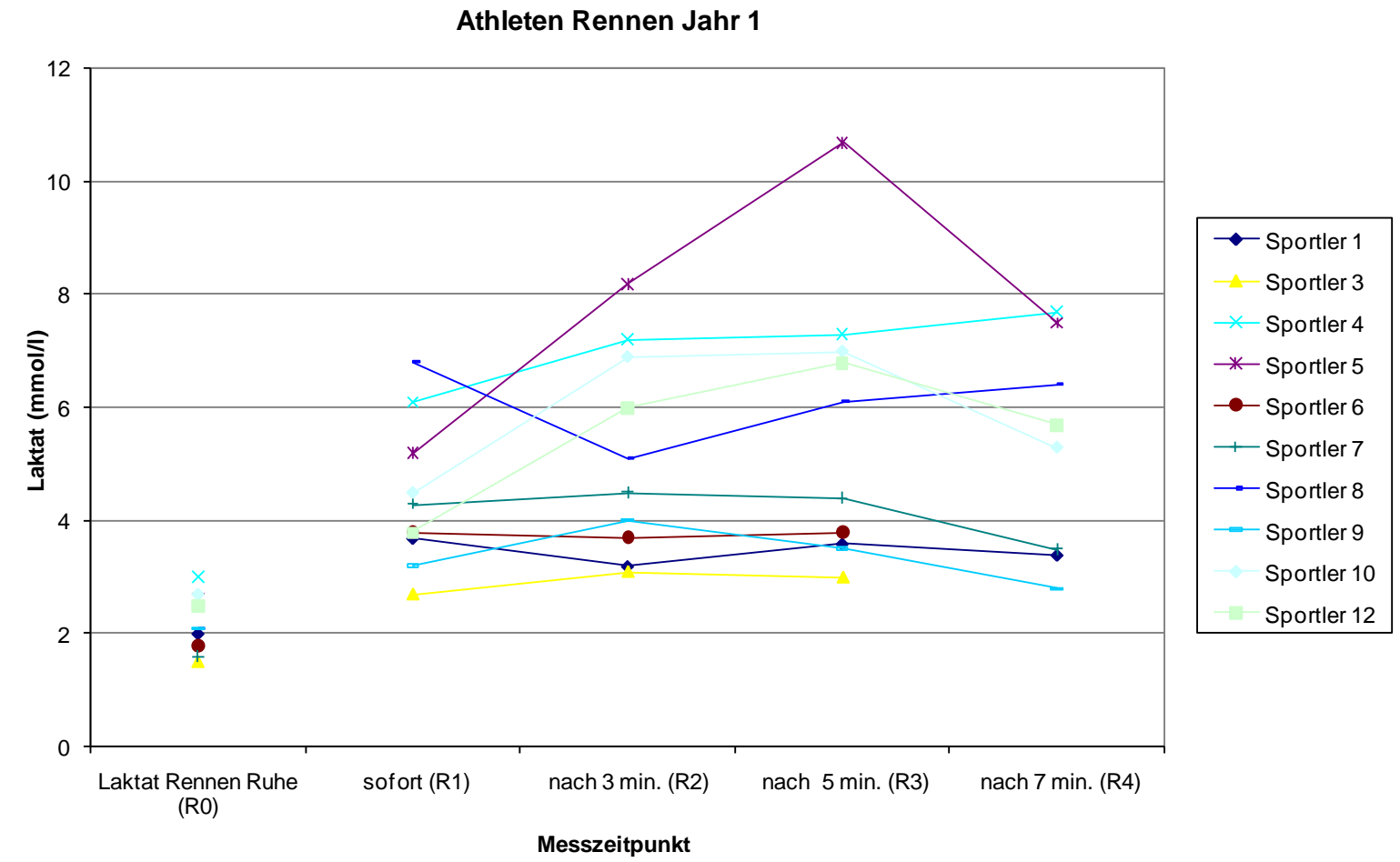

Abbildung 4: Rennen Jahr 1 - Alle Athleten 
In den vorliegenden Abbildungen steigen die Kurven der Athleten 4, 5, 8, 10 und 12 stärker an, das Niveau der Maximalwerte ist höher angesiedelt als bei den anderen Athleten. Weiterhin lassen sich die Verläufe der Kurven von Sportler 5 und 8 mit den anderen Kurven kaum vergleichen, die Ausschläge der Werte innerhalb der Kurven sind deutlich höher.

\subsubsection{Jahr 2}

Tabelle 3: Rennen Jahr 2 - Alle Athleten

$$
\text { Laktat }(\mathrm{mmol} / \mathrm{l})
$$

\begin{tabular}{|c|c|c|c|c|c|}
\hline Sportler Jahr 2 & Laktat Rennen Ruhe (R0) & sofort (R1) & nach 3 min. (R2) & nach 5 min. (R3) & nach 7 min. (R4) \\
\hline Sportler 1 & 1,8 & 2,3 & 2,9 & 1,9 & \\
\hline Sportler 3 & 1,9 & 2,2 & 3,2 & 3,5 & \\
\hline Sportler 4 & 2,2 & 3,1 & 5,6 & 3,3 & \\
\hline Sportler 5 & 3,3 & 7,8 & 7,3 & 8,7 & \\
\hline Sportler 6 & 2,0 & 3,6 & 3,1 & 3,1 & \\
\hline Sportler 7 & 2,1 & 5,1 & 4,5 & 3,3 & \\
\hline Sportler 8 & 2,0 & 4,5 & 7,1 & 6,6 & \\
\hline Sportler 10 & 1,7 & Disqu. & & & \\
\hline Sportler 11 & 2,4 & 7,2 & 3,2 & 3,5 & \\
\hline Sportler 13 & 3,2 & 2,3 & 3,3 & 3,7 & \\
\hline
\end{tabular}

Im zweiten Jahr wurden ebenfalls bei zehn Sportlern Laktatmessungen vorgenommen. Ein Athlet wurde disqualifiziert, so dass keine Messergebnisse nach R0 vorliegen. Bei allen anderen zeigt sich - wie bereits im Vorjahr - ein Anstieg der Werte zwischen den Zeitpunkten R0 und R1. Maximalwerte variieren stark, die Athleten 4, 5, 7, 8 und 11 verzeichnen höhere Maximalwerte, während die Sportler 1, 3, 6 und 13 auch zum Zeitpunkt der maximal gemessenen Laktatkonzentration eine Konzentration von $4 \mathrm{mmol} / \mathrm{l}$ nicht überschreiten. Es liegen keine Messwerte zum Zeitpunkt R4 vor. 


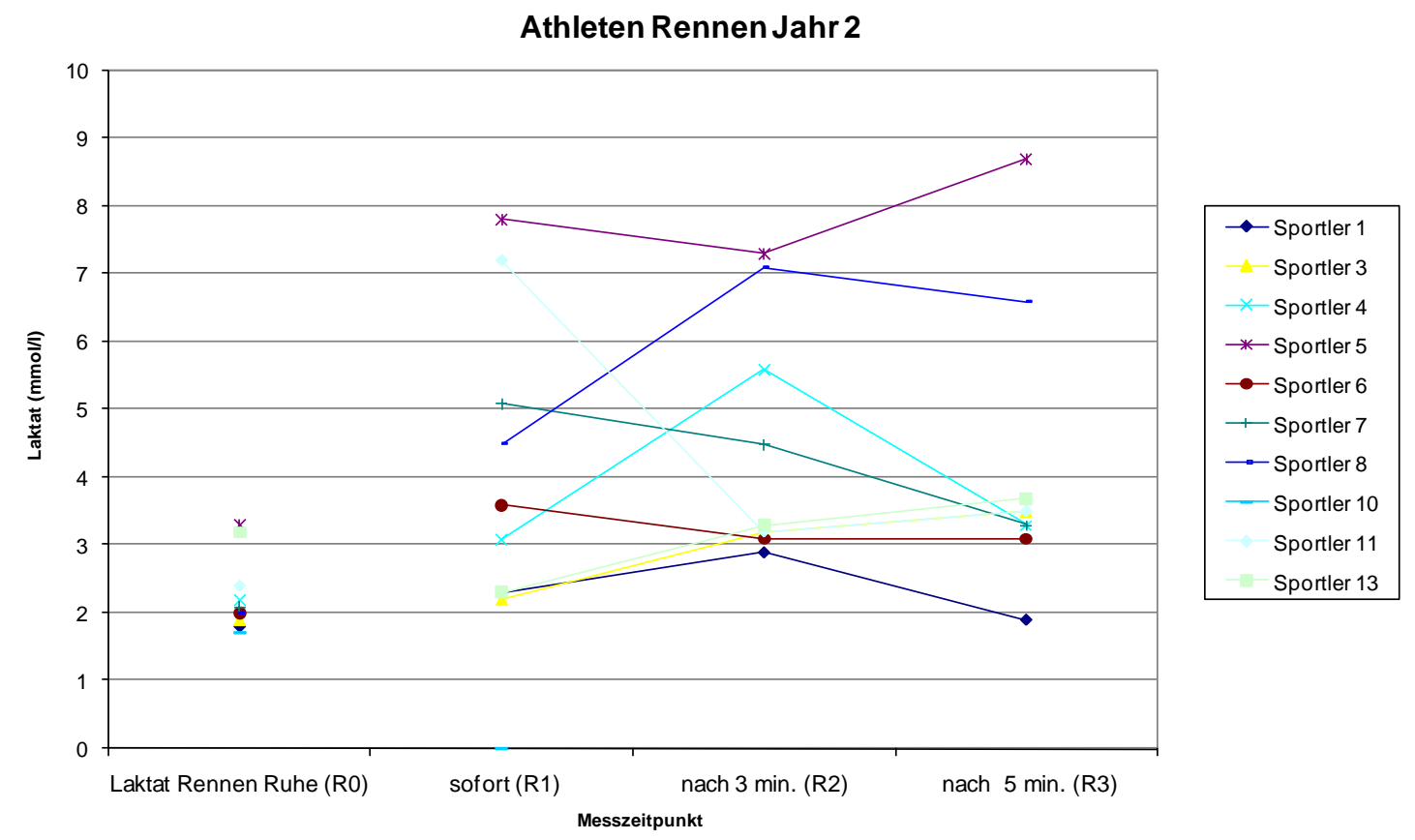

Abbildung 5: Rennen Jahr 2 - Alle Athleten

Die Kurvenverläufe im zweiten Jahr der Messungen zeigen keine Regelmäßigkeiten, die Ausschläge der Kurven sind größer als bei der Messung in Jahr 1.

\subsubsection{Jahr 3 (1. Messung)}

Tabelle 4: Rennen Jahr 3 (1. Messung) - Alle Athleten

Laktat $(\mathrm{mmol} / \mathrm{l})$

\begin{tabular}{lccccc} 
Sportler Jahr 3 (I.) & Ruhe (R0) & sofort (R1) & nach 3 min. (R2) & nach 5 min. (R3) & nach 7 min. (R4) \\
\hline Sportler 1 & 1,8 & 3,6 & 2,4 & 2,6 & 2,3 \\
Sportler 2 & 2,2 & 5,0 & 4,5 & 4,1 & 3,3 \\
Sportler 4 & 1,5 & 9,5 & 8,2 & 6,9 & 7,0 \\
Sportler 5 & 1,7 & 10,4 & 9,2 & 9,8 & 9,6 \\
Sportler 6 & 1,7 & 5,4 & 5,4 & 5,1 & 4,6 \\
Sportler 7 & 1,4 & 3,8 & 4,7 & 4,7 & 3,6 \\
Sportler 14 & 1,7 & 6,9 & 6,6 & 5,2 & 4,3
\end{tabular}

Im dritten Jahr nahmen an beiden Messreihen sieben Sportler teil.

In der Tabelle, welche die Daten der ersten Messung wiedergibt, zeigt sich, dass die Laktatkonzentration bei allen Athleten nach R0 ansteigt. Sportler 1 und 7 erreichen Werte unterhalb einer Konzentration von $5 \mathrm{mmol} / \mathrm{l}$, die anderen Sportler erreichen vereinzelt deutlich höhere Werte. 


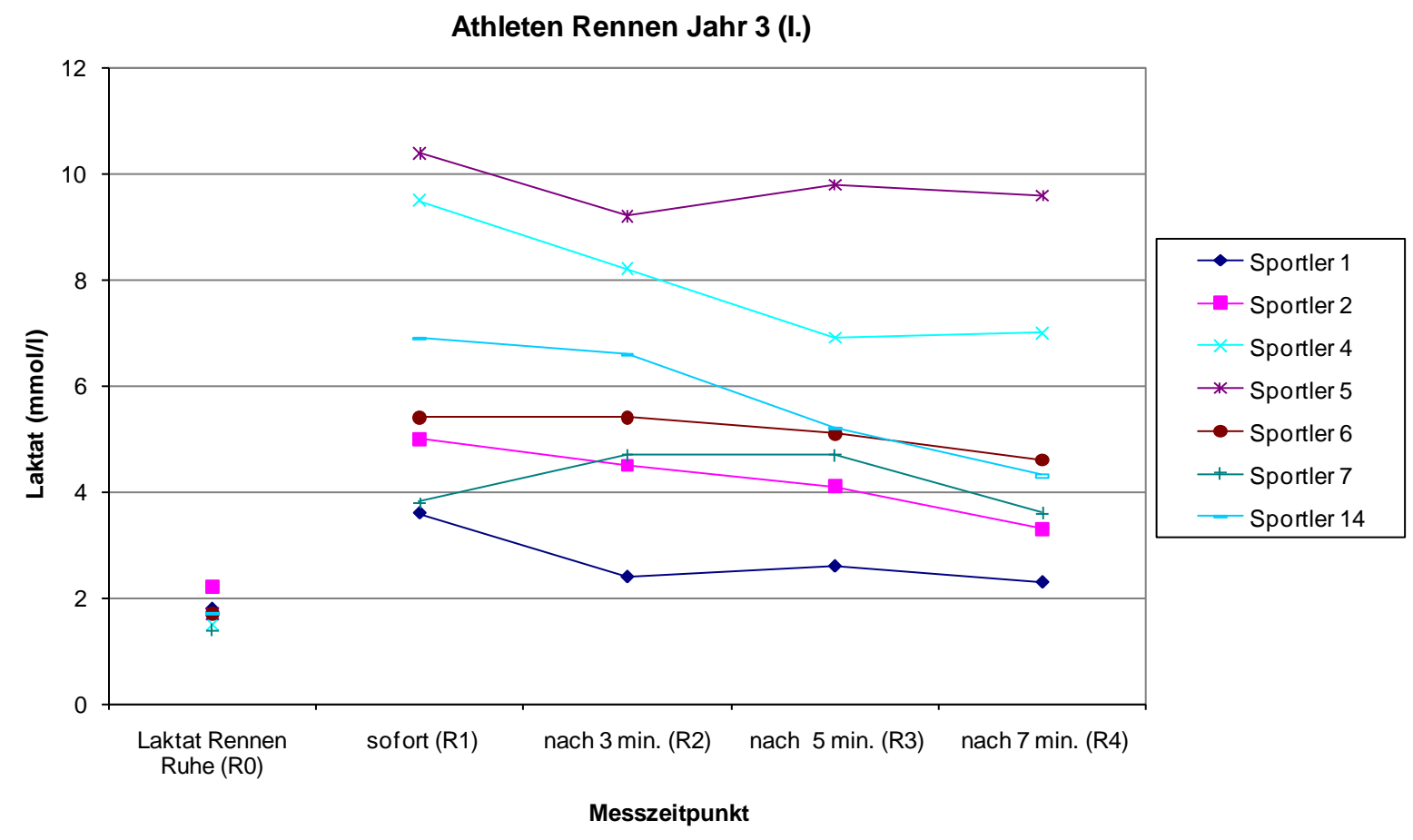

Abbildung 6: Rennen Jahr 3 (1. Messung) - Alle Athleten

In den Abbildungen sind die Konzentrationsverlaufskurven sehr ähnlich: Sie verlaufen nahezu parallel.

\subsubsection{Jahr 3 (2. Messung)}

Tabelle 5: Rennen Jahr 3 (2. Messung) - Alle Athleten

Laktat $(\mathrm{mmol} / \mathrm{l})$

\begin{tabular}{lccccc} 
Sportler Jahr 3 (II.) & Ruhe (R0) & sofort (R1) & nach 3 min. (R2) & nach 5 min. (R3) & nach 7 min. (R4) \\
\hline Sportler 1 & 1,4 & 3,0 & 2,3 & 2,2 & 2,0 \\
Sportler 2 & 2,4 & 3,7 & 3,9 & 3,2 & 2,6 \\
Sportler 4 & 2,0 & 10,2 & 7,6 & 6,8 & 6,7 \\
Sportler 5 & 2,0 & 8,1 & 7,1 & 7,5 & 6,6 \\
Sportler 6 & 1,9 & 6,2 & 5,7 & 4,9 & 3,8 \\
Sportler 7 & 1,6 & 4,6 & 4,8 & 2,8 & 2,5 \\
Sportler 14 & 1,7 & 3,8 & 4,0 & 3,9 & 3,0
\end{tabular}

In der zweiten Messreihe aus dem dritten Jahr zeigen sich ähnliche Ergebnisse wie in der ersten Messreihe. Die Laktatkonzentrationen bei den Sportlern 1, 2 und 14 überschreiten die Konzentration von $4 \mathrm{mmol} / \mathrm{l}$ nicht, während einige Athleten Werte aufweisen, die mehr als das Zweifache betragen. Annähernd alle Athleten erreichen die maximale Laktatkonzentration zum Zeitpunkt R1. 


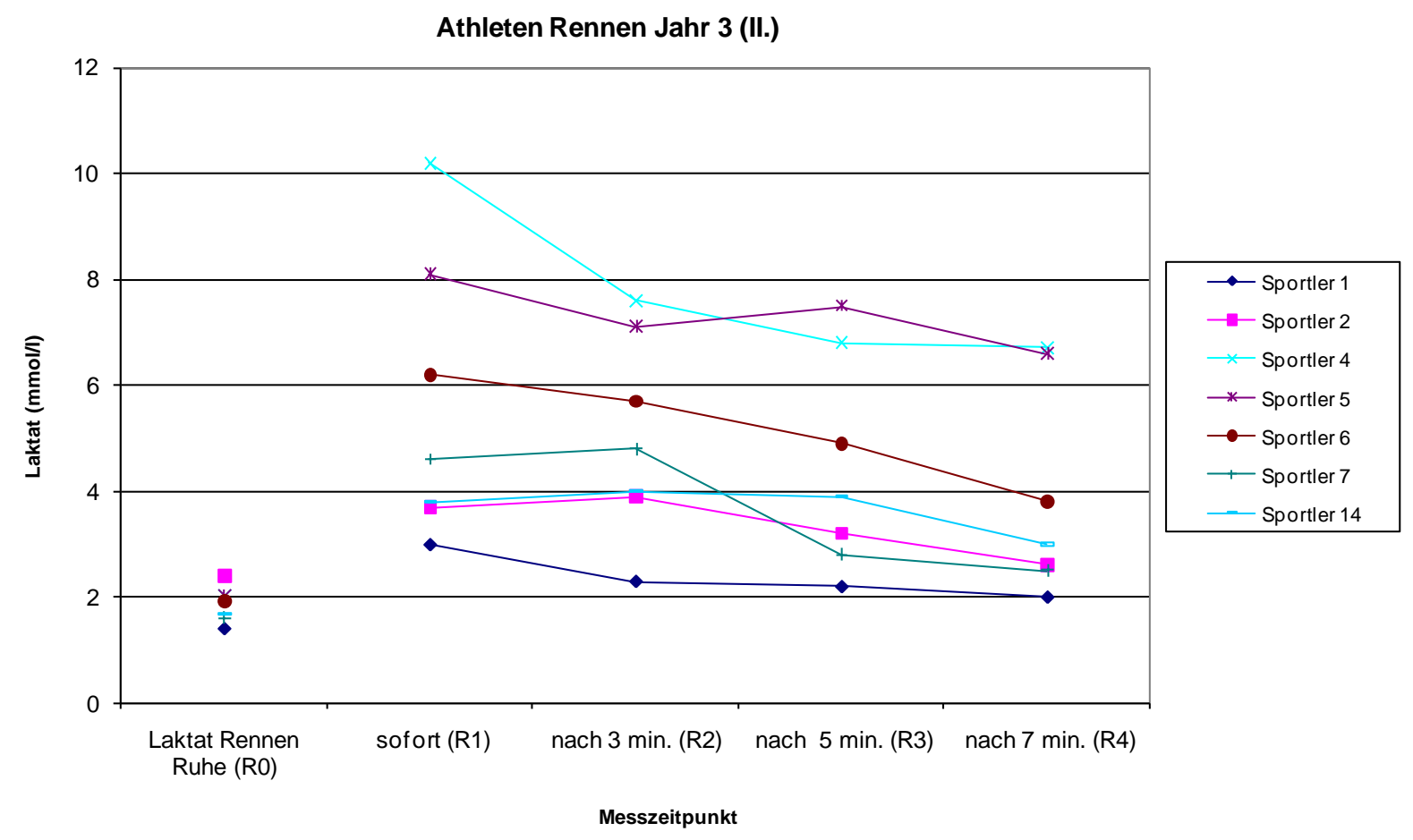

Abbildung 7: Rennen Jahr 3 (2. Messung) - Alle Athleten

Auf Grund der unterschiedlichen technischen Ausführung des Skifahrens wird die Probandengruppe zur Auswertung der Ergebnisse in Sitzend und Stehend unterteilt. 


\subsection{Sitzende und stehende Sportler - Einzelfallbetrachtung}

In der folgenden Abbildung sind die mittleren Laktatkonzentrationskurven von sitzenden und stehenden Athleten, gebildet aus allen Einzelwerten innerhalb der jeweiligen Gruppe, nach Belastung in Wettkampf und Training dargestellt.

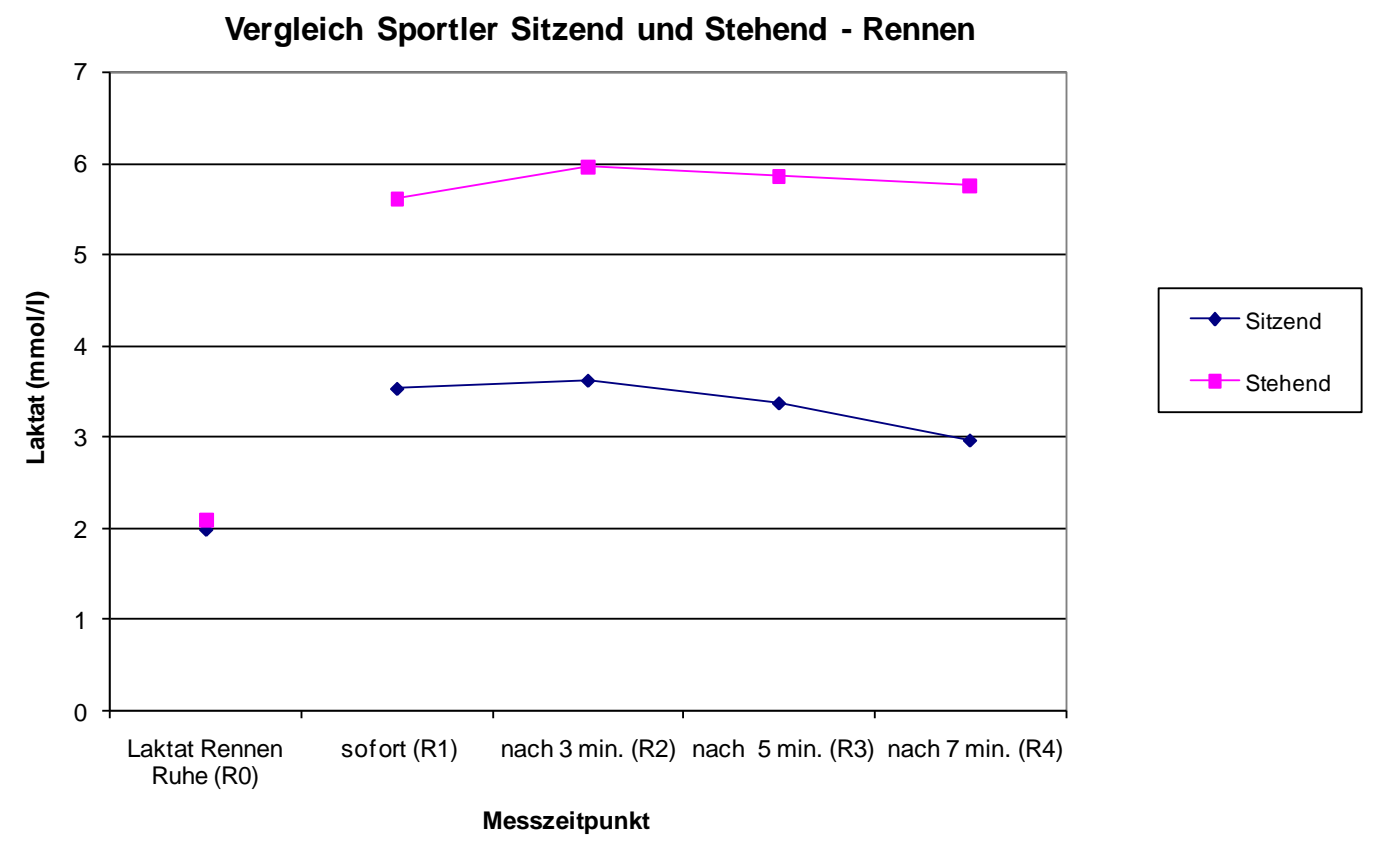

Abbildung 8: Athleten Rennen insgesamt - Sitzend und Stehend

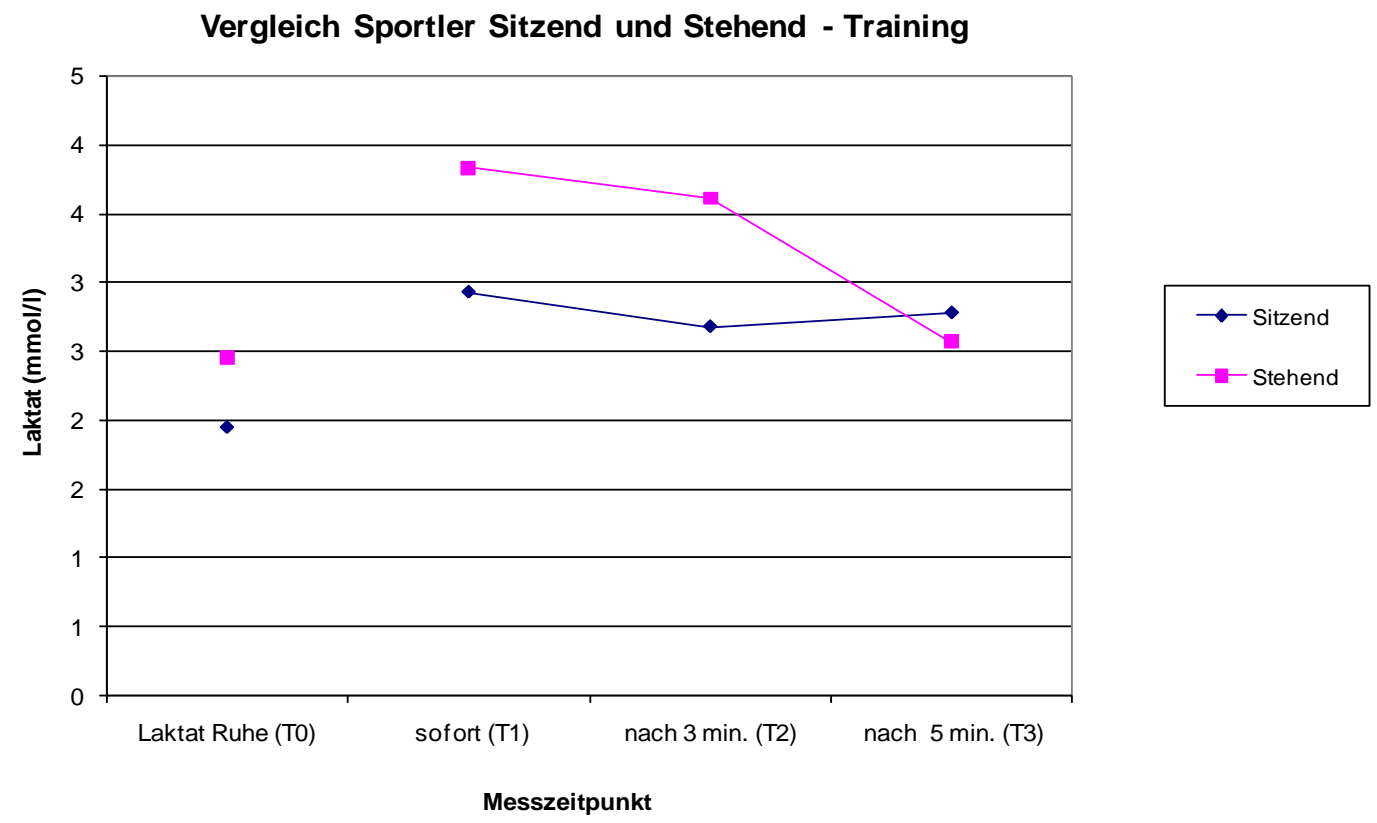

Abbildung 9: Athleten Training insgesamt - Sitzend und Stehend 
Bei Betrachtung der Einzelwerte wird deutlich, dass die Messergebnisse in einem großen Bereich um den Mittelwert streuen; sowohl in der gesamten Probandengruppe als auch in den Untergruppen Sitzend und Stehend.

Die Sportler sind in unterschiedlicher Form physisch eingeschränkt, woraus sich eine jeweils individuelle technische Umsetzung des Skifahrens ergibt. Auf Grund dessen erfolgt zunächst eine Einzelfallbetrachtung der Athleten.

\subsubsection{Sitzende Sportler}

\subsubsection{1 Überblick der Wettkampfmessergebnisse Jahr 1 bis 3}

In Abbildung 10 sind die mittleren Konzentrationskurven aus allen Reihen der Wettkampfmessungen der sitzenden Sportler dargestellt. Im Anschluss werden die Daten entsprechend den einzelnen Jahren aufgeschlüsselt.

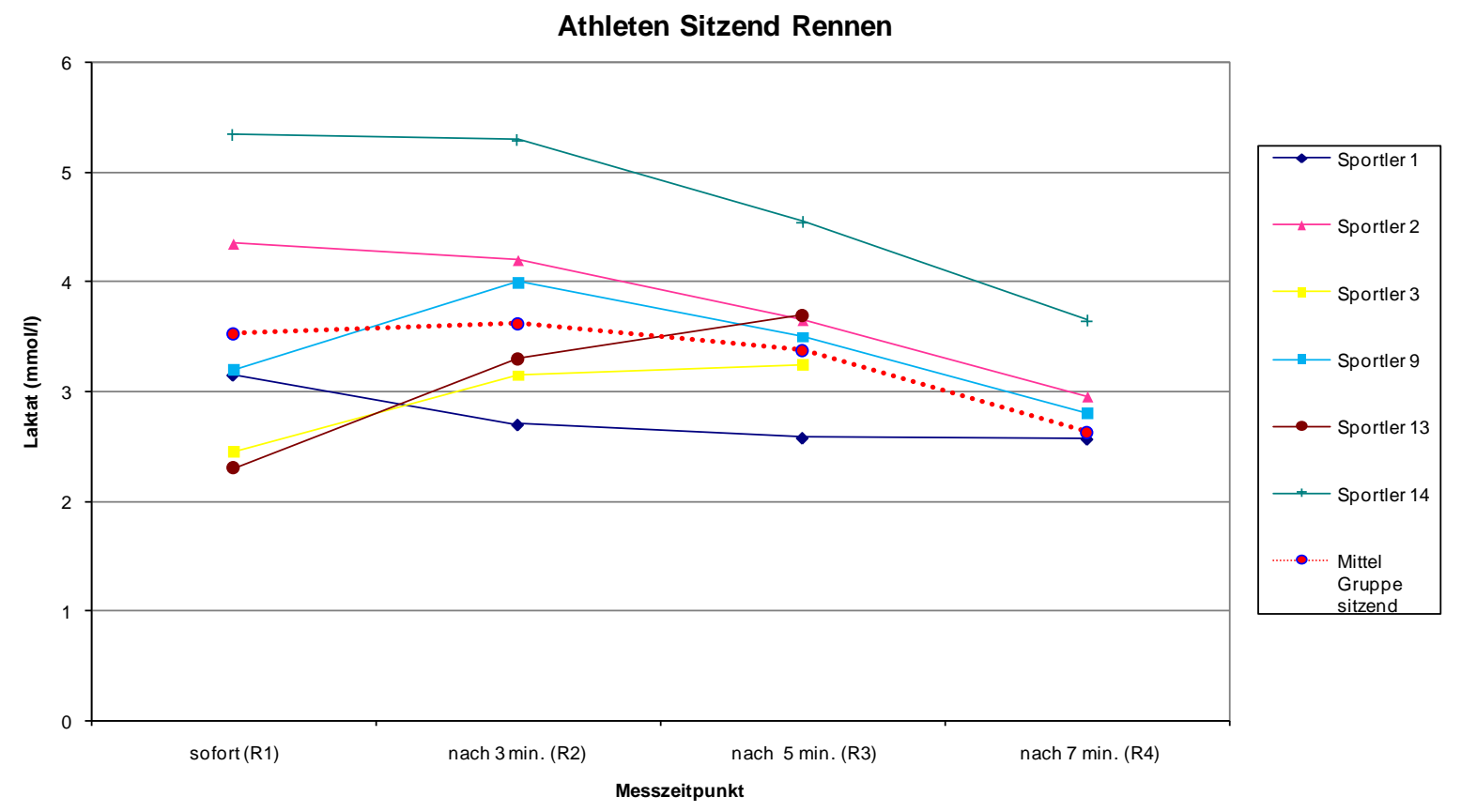

Abbildung 10: Athleten Sitzend Rennen gesamt 


\subsection{Jahr 1}

Tabelle 6: Rennen Jahr 1 - Sitzende Sportler

Laktat (mmol/l)

Sportler Jahr 1 Sitzend Ruhe (R0)

Sportler 1

2,0

sofort (R1)

nach 3 min. (R2)

nach $5 \min .(R 3)$

nach $7 \mathrm{~min} .(\mathrm{R} 4)$

Sportler 3

1,5

3,7

3,2

3,6

3,4

Sportler 9

2,1

2,7

3,1

3,0

$3,2 \quad 4,0$

3,5

2,8

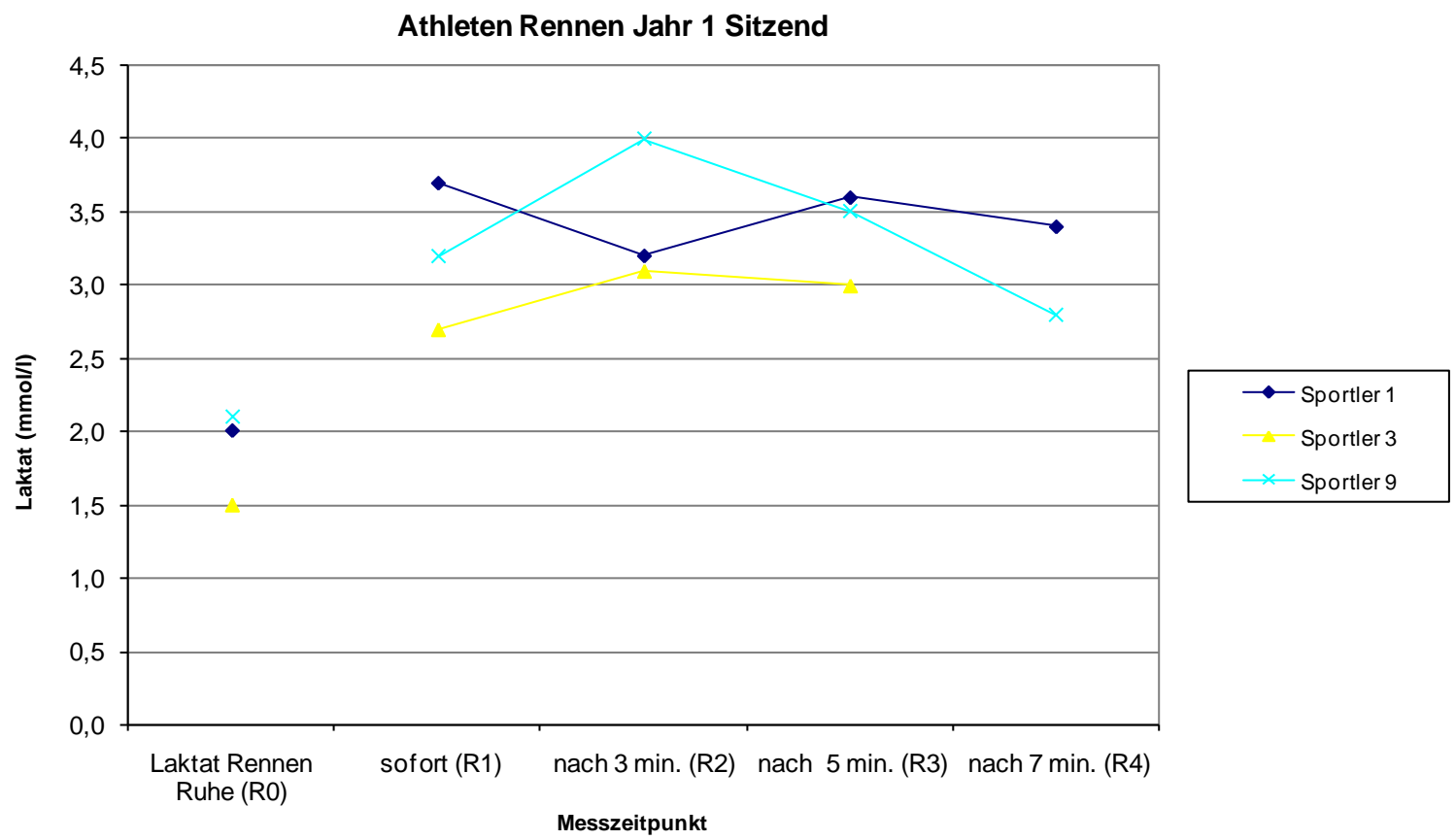

Abbildung 11: Rennen Jahr 1 - Sitzende Sportler 


\subsection{Jahr 2}

Tabelle 7: Rennen Jahr 2 - Sitzende Sportler

Laktat $(\mathrm{mmol} / \mathrm{l})$

\begin{tabular}{lcccc} 
Sportler Jahr 2 Sitzend & Ruhe (R0) & sofort (R1) & nach 3 min. (R2) & nach 5 min. (R3) \\
\hline Sportler 1 & 1,8 & 2,3 & 2,9 & 1,9 \\
Sportler 3 & 1,9 & 2,2 & 3,2 & 3,5 \\
Sportler 13 & 3,2 & 2,3 & 3,3 & 3,7
\end{tabular}

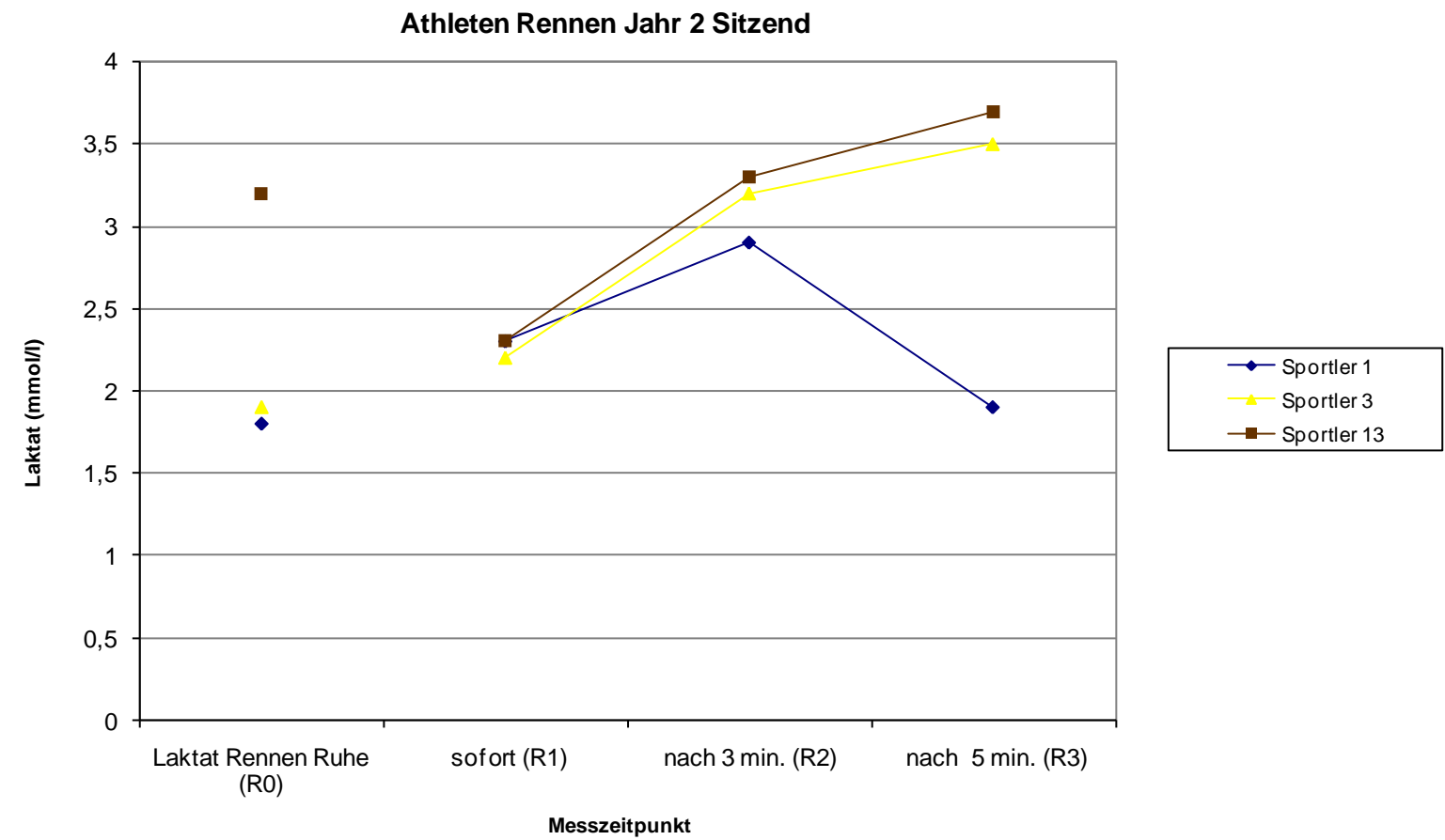

Abbildung 12: Rennen Jahr 2 - Sitzende Sportler

\subsection{Jahr 3 (1. Messung)}

Tabelle 8: Rennen Jahr 3 (I.) - Sitzende Sportler

Laktat $(\mathrm{mmol} / \mathrm{l})$

\begin{tabular}{lccccc} 
Sportler Jahr 3 (I.) Sitzend & Ruhe (R0) & sofort (R1) & nach 3 min. (R2) & nach 5 min. (R3) & nach 7 min. (R4) \\
\hline Sportler 1 & 1,8 & 3,6 & 2,4 & 2,6 & 2,3 \\
Sportler 2 & 2,2 & 5,0 & 4,5 & 4,1 & 3,3 \\
Sportler 14 & 1,7 & 6,9 & 6,6 & 5,2 & 4,3
\end{tabular}




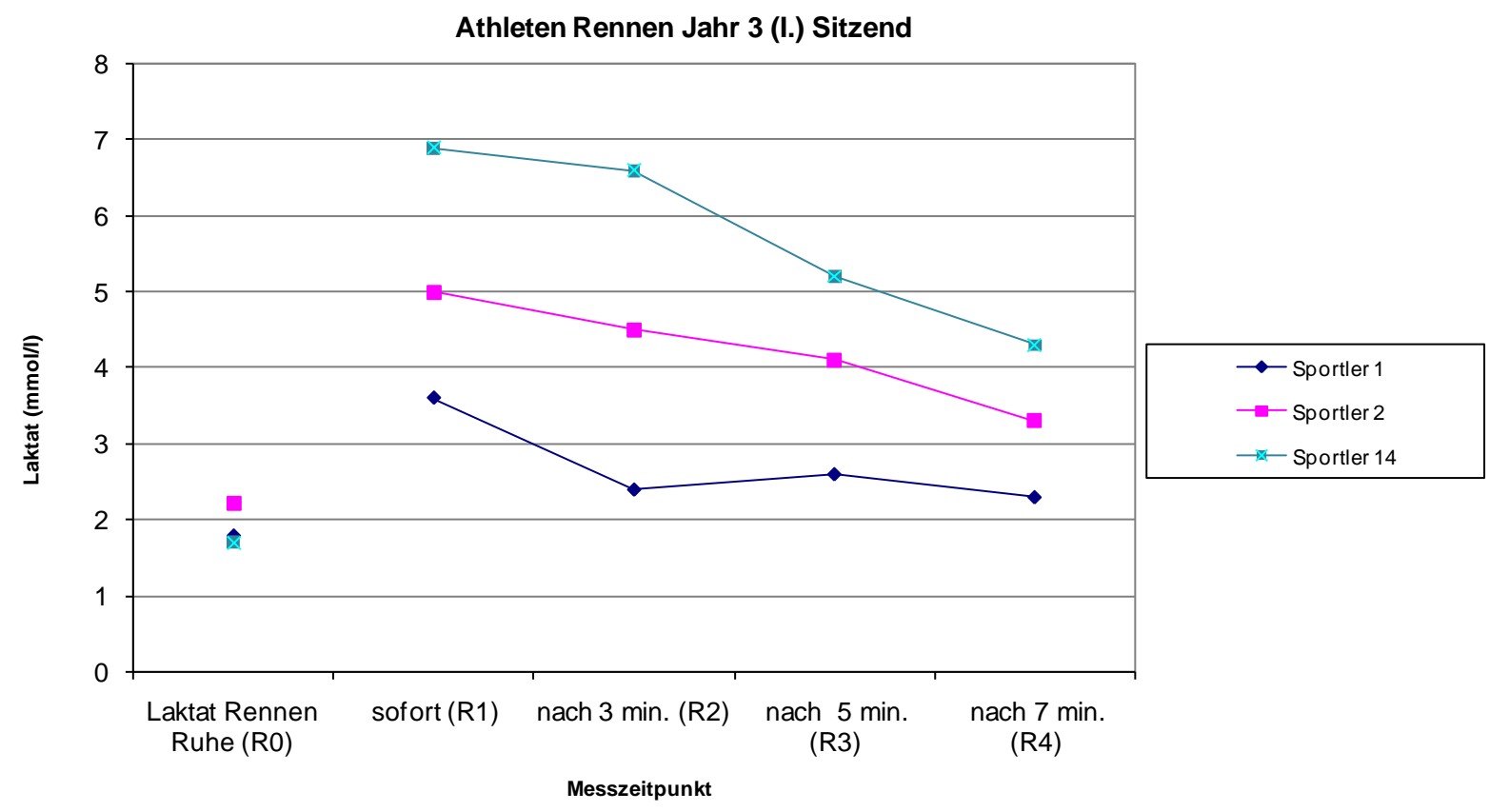

Abbildung 13: Rennen Jahr 3 (I.) - Sitzende Sportler

\subsection{Jahr 3 (2. Messung)}

Tabelle 9: Rennen Jahr 3 (II.) - Sitzende Sportler

Laktat $(\mathrm{mmol} / \mathrm{l})$

\begin{tabular}{lccccc} 
Sportler Jahr 3 (II.) Sitzend & Ruhe (R0) & sofort (R1) & nach 3 min. (R2) & nach 5 min. (R3) & nach 7 min. (R4) \\
\hline Sportler 1 & 1,4 & 3,0 & 2,3 & 2,2 & 2,0 \\
Sportler 2 & 2,4 & 3,7 & 3,9 & 3,2 & 2,6 \\
Sportler 14 & 1,7 & 3,8 & 4,0 & 3,9 & 3,0
\end{tabular}




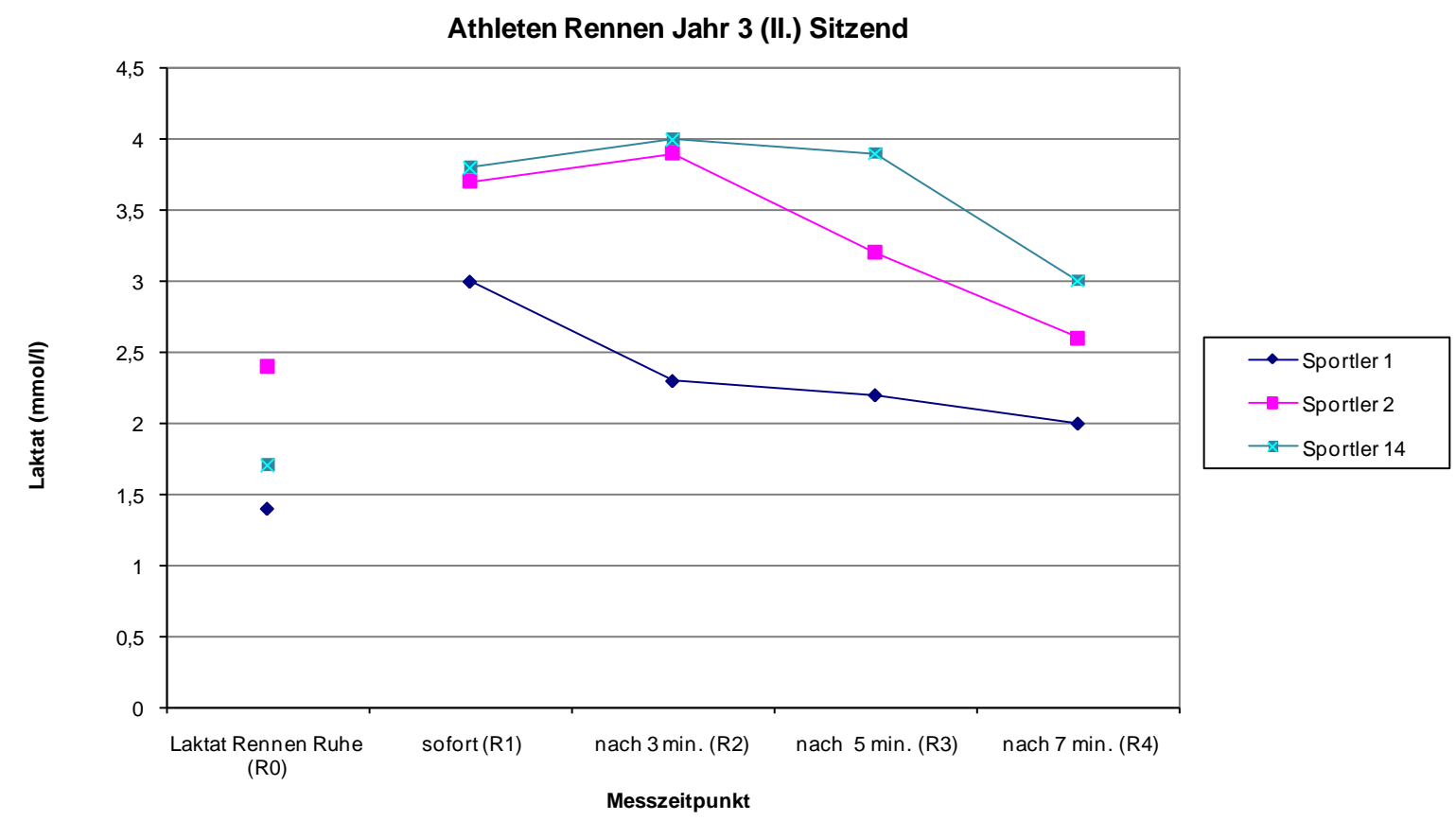

Abbildung 14: Rennen Jahr 3 (II.) - Sitzende Sportler

Die Ruhewerte der sitzenden Sportler im Rennen in Jahr 1 liegen im Mittel bei 1,87 mmol/l; maximal werden Laktatwerte von 3,1 bis $4 \mathrm{mmol} / \mathrm{l}$ erreicht.

In Jahr 2 liegen die Ruhwerte zwischen 1,8 und 3,2 mmol/l (Mittelwert 2,3 $\mathrm{mmol} / \mathrm{l}$ ), die Athleten erreichen Maximalwerte zwischen 2,9 und 3,7 mmol/l.

In Jahr 3 sind die Ruhewerte der Laktatkonzentration der sitzenden Sportler in der ersten Messreihe zwischen 1,7 und 2,2 $\mathrm{mmol} / \mathrm{l}$ (Mittelwert $1,9 \mathrm{mmol} / \mathrm{l}$ ) angesiedelt, maximal erreichen sie Werte von 3,6 bis 6,9 mmol/l. In der zweiten Messreihe beträgt der Mittelwert der in Ruhe gemessenen Laktatwerte 1,83 mmol/1, die Maximalwerte liegen zwischen 3,0 und $4,0 \mathrm{mmol} / 1$.

Die leistungsdiagnostischen Untersuchungen während der Vorbereitungsphase im Sommer wurden bei den sitzenden Sportlern mit Hilfe eines Handbikes durchgeführt, das auf einem Laufband gefahren wird.

Über die Einstellung der Geschwindigkeit des Laufbandes wurden Belastungsstufen festgelegt. Von besonderem Interesse sind die Nachbelastungswerte, da diese auch im Winter betrachtet werden. 


\subsubsection{Sportler 1}

\subsection{Rennen}

Tabelle 10: Sportler 1 - Rennen Jahre 1 bis 3

\begin{tabular}{|c|c|c|c|c|c|}
\hline Sportler 1 & $\begin{array}{l}\text { Laktat Rennen } \\
\text { Ruhe (R0) }\end{array}$ & sofort (R1) & nach 3 min. (R2) & nach 5 min. (R3) & nach 7 min. (R4) \\
\hline$\overline{\text { Rennen RS Jahr } 1}$ & 2,0 & 3,7 & 3,2 & 3,6 & 3,4 \\
\hline Rennen RS Jahr 2 & 1,8 & 2,3 & 2,9 & 1,9 & \\
\hline Rennen RS Jahr 3 (I.) & 1,8 & 3,6 & 2,4 & 2,6 & 2,3 \\
\hline Rennen RS Jahr 3 (II.) & 1,4 & 3,0 & 2,3 & 2,2 & 2,0 \\
\hline Mittelwert zum Messzeitpunkt & 1,75 & 3,15 & 2,70 & 2,58 & 2,57 \\
\hline Standardabweichung & 0,25 & 0,65 & 0,42 & 0,74 & 0,74 \\
\hline
\end{tabular}

\subsection{Training}

Tabelle 11: Sportler 1 - Training Jahr 1 und 2

\begin{tabular}{lllll}
\multicolumn{2}{l}{$\begin{array}{l}\text { Laktat Training } \\
\text { Sportler 1 }\end{array}$} & $\begin{array}{l}\text { sofort (T1) } \\
\text { Ruhe) }\end{array}$ & nach 3 min. (T2) & nach 5 min. (T3) \\
\hline Training Jahr 1 & 1,8 & 3,3 & 2,9 & 2,3 \\
\hline Training Jahr 2 & 1,6 & 3,3 & 1,8 & 1,9 \\
\hline Mittelwert zum Messzeitpunkt & 1,7 & 3,3 & 2,35 & 2,1 \\
\hline Standardabweichung & 0,14 & 0,00 & 0,78 & 0,28 \\
\hline
\end{tabular}

Bei einem Vergleich der drei Wettkampfjahre zeigt sich bei Sportler 1 ein mittlerer Konzentrationswert des Laktats im Blut von $1,75 \mathrm{mmol} / \mathrm{l}$ zum Zeitpunkt R0. In drei Messungen wird der Maximalwert zum Zeitpunkt R1 erreicht; die Kurven zeigen in allen Jahren einen Abfall nach Erreichen der maximalen Laktatkonzentration.

Im Training liegt der mittlere Wert zum Zeitpunkt T0 bei $1,7 \mathrm{mmol} / \mathrm{l}$, der Maximalwert wird bei T1 erreicht, die Steigung nach Erreichen des Maximalpunktes ist negativ.

\subsection{Vorbereitungsphase Sommer}

Tabelle 12: Sportler 1 - Messergebnisse Sommer

\begin{tabular}{lllll} 
& \multicolumn{2}{l}{ Laktat in Ruhe } & & \\
Sportler 1 & (S0) & sofort (S1) & nach 3 min. (S2) & nach 5 min. (S3) \\
\hline Sommer 1 & 0,78 & 13,1 & 13,9 & 13,9 \\
\hline Sommer 2 & 1,5 & 10,1 & 10,8 & 10,3 \\
\hline Sommer 3 & 1,3 & 11,3 & 12,2 & 1,0 \\
\hline & & & & 11,73 \\
\hline Mittelwert zum Messzeitpunkt & 1,19 & 11,5 & 12,3 & 1,91
\end{tabular}

Die Werte der Laktatkonzentration im Blut in den Sommermessungen nach Ergometerbelastung sind deutlich höher als jene im Winter erhobenen Nachbelastungswerte; die Ruhewerte betragen im Mittel 1,19 mmol/l Blut. Die Differenzen der 
Laktatkonzentrationen zwischen den Zeitpunkten S0 und S1 betragen im Mittelwert 10,31 mmol/l. Zwischen S1 und S2 zeigt sich eine geringe Zunahme der Laktatkonzentration, während sie zum Zeitpunkt S3 abfällt.

\subsubsection{Sportler 2}

\subsection{Rennen}

Tabelle 13: Sportler 2 - Rennen Jahre 1 bis 3

\begin{tabular}{llllll} 
Sportler 2 & R0 & R1 & R2 & R3 & \\
\hline Rennen RS Jahr 1 & & & & \\
\hline Rennen RS Jahr 2 & & & 4,5 & 4,1 & \\
\hline Rennen RS Jahr 3 (I.) & 2,2 & 5,0 & 3,9 & 3,2 & \\
\hline Rennen RS Jahr 3 (II.) & 2,4 & 3,7 & & & \\
\hline & & & 4,2 & 3,65 & 2,6 \\
\hline Mittelwert zum Messzeitpunkt & 2,3 & 4,35 & 0,42 & 0,64 & 0,49 \\
\hline Standardabweichung & 0,14 & 0,92 & & \\
\hline
\end{tabular}

Im Mittel beträgt die Laktatkonzentration zum Zeitpunkt R0 2,3 mmol/l Blut, ein Maximum wird in der ersten Messreihe zum Zeitpunkt R1, in der zweiten bei R2 erreicht. In beiden Jahren fallen die Konzentrationskurven nach Erreichen des Maximalwertes ab.

\subsection{Vorbereitungsphase Sommer}

Tabelle 14: Sportler 2 - Messergebnisse Sommer

\begin{tabular}{lllll} 
Sportler 2 & S0 & S1 & S2 & S3 \\
\hline Sommer 1 & 1,18 & 10,8 & 10,6 & 10,9 \\
\hline Sommer 2 & & & & \\
\hline Sommer 3 & & & & 10,90 \\
\hline & & 10,8 & 10,6 &
\end{tabular}

Im Sommer zeigen sich unter bzw. nach Belastung Laktatkonzentrationen im Blut, die deutlich höher sind als jene, die während der Wintermessungen erhoben wurden. Der Ruhewert liegt bei $1,18 \mathrm{mmol} / \mathrm{l}$. Nach Belastungsende steigen die Konzentrationen des Laktats bis zum Zeitpunkt R3 bis maximal 10,9 mmol/l an. Eine Aussage über das Steigungsverhalten der Kurve nach dem Maximalwert ist nicht möglich, da erst zum Zeitpunkt S3 ein Maximum erreicht wird. 


\subsubsection{Sportler 3}

\subsection{Rennen}

Tabelle 15: Sportler 3 - Rennen Jahre 1 bis 3

\begin{tabular}{|c|c|c|c|c|c|}
\hline Sportler 3 & Ro & $\mathrm{R} 1$ & $\mathrm{R} 2$ & R3 & $\mathrm{R} 4$ \\
\hline Rennen RS Jahr 1 & 1,5 & 2,7 & 3,1 & 3,0 & \\
\hline Rennen RS Jahr 2 & 1,9 & 2,2 & 3,2 & 3,5 & \\
\hline \multicolumn{6}{|l|}{ Rennen RS Jahr 3 (I.) } \\
\hline \multicolumn{6}{|l|}{ Rennen RS Jahr 3 (II.) } \\
\hline Mittelwert zum Messzeitpunkt & 1,7 & 2,45 & 3,15 & 3,25 & \\
\hline Standardabweichung & 0,28 & 0,35 & 0,07 & 0,35 & \\
\hline
\end{tabular}

\subsection{Training}

Tabelle 16: Sportler 3 - Training Jahr 1 und 2

\begin{tabular}{lcccc} 
Sportler 3 & T0 & T1 & T2 & T3 \\
\hline Training Jahr 1 & & & & 3,0 \\
\hline Training Jahr 2 & 1,8 & 3,0 & 2,8 & 3,0 \\
\hline Mittelwert zum Messzeitpunkt & 1,8 & 3,0 & 2,8 & \\
\hline Standardabweichung & & & & \\
\hline
\end{tabular}

Insgesamt liegt die mittlere Laktatkonzentration zum Zeitpunkt R0 bei $1,7 \mathrm{mmol} / \mathrm{l}$. Ein Maximum wird bei R2 oder R3 erreicht, so dass nur im ersten Jahr der Messungen eine Aussage über den Kurvenverlauf nach Erreichen des Maximums getroffen werden kann. Die Steigung ist negativ, die Kurve fällt leicht ab.

Im Training liegt nur eine Messreihe vor, die Maximalkonzentration beträgt bei T1 3,0 mmol Laktat/l Blut, die Kurve fällt anschließend leicht ab und steigt erneut an.

\subsection{Vorbereitungsphase Sommer}

Tabelle 17: Sportler 3 - Messergebnisse Sommer

\begin{tabular}{lllll} 
Sportler 3 & S0 & S1 & S2 & S3 \\
\hline Sommer 1 & 0,79 & 13,99 & 14,23 & 13,61 \\
\hline Sommer 2 & 2,1 & 12,5 & 11,6 & 12,2 \\
\hline Sommer 3 & 1,6 & 11,0 & 11,4 & 10,2 \\
\hline & & & & \\
\hline Mittelwert zum Messzeitpunkt & 1,50 & 12,50 & 12,41 & 12,00 \\
\hline Standardabweichung & 0,66 & 1,50 & 1,58 & 1,71
\end{tabular}

In der Sommermessung zeigt sich ein mittlerer Konzentrationswert von 1,497 mmol/l zum Zeitpunkt S0, die Laktatkonzentration steigt bis S1 signifikant an. Im ersten und dritten Jahr der Sommermessungen wird der maximale Konzentrationswert drei Minuten nach Belastungsende (S2) erreicht, anschließend fällt die Laktatkonzentration ab. In Sommer 2 wird das Maximum bei S1 erreicht, die Kurve fällt bis S2 ab und steigt erneut an bis zum Zeitpunkt S3, erreicht jedoch nicht den Maximalwert. 


\subsubsection{Sportler 9}

\subsection{Rennen}

Tabelle 18: Sportler 9 - Rennen Jahre 1 bis 3

\begin{tabular}{|c|c|c|c|c|c|}
\hline Sportler 9 & Ro & $\mathrm{R} 1$ & $\mathrm{R} 2$ & R3 & $\mathrm{R} 4$ \\
\hline Rennen RS Jahr 1 & 2,1 & 3,2 & 4,0 & 3,5 & 2,8 \\
\hline \multicolumn{6}{|l|}{ Rennen RS Jahr 2} \\
\hline \multicolumn{6}{|l|}{ Rennen RS Jahr 3 (I.) } \\
\hline \multicolumn{6}{|l|}{ Rennen RS Jahr 3 (II.) } \\
\hline Mittelwert zum Messzeitpunkt & 2,1 & 3,2 & 4,0 & 3,50 & 2,8 \\
\hline Standardabweichung & & & & & \\
\hline
\end{tabular}

Das Maximum der Laktatkonzentration wird zum Zeitpunkt R2 nach drei Minuten erreicht (4 mmol/l), anschließend fällt die Konzentration allmählich ab.

\subsection{Vorbereitungsphase Sommer}

Tabelle 19: Sportler 9 - Messergebnisse Sommer

\begin{tabular}{llllll} 
Sportler 9 & S0 & $30 \mathrm{~W}$ & $60 \mathrm{~W}$ & $90 \mathrm{~W}$ & $120 \mathrm{~W}(\mathrm{~S} 1)$ \\
\hline Sommer 1 & 0,88 & 2,8 & 4,86 & 7,63 & 13,3 \\
\hline Sommer 2 & & & & & \\
\hline Sommer 3 & & & & & \\
\hline & & & & & \\
\hline Mittelwert zum Messzeitpunkt & 0,88 & 2,8 & 4,86 & 7,63 &
\end{tabular}

Die erste Belastungsstufe während der Untersuchungen auf dem Ergometer im Sommer beträgt 30 Watt, die Steigerung der Belastung erfolgt um jeweils 30 Watt. Vor Belastung wird eine Laktatkonzentration von $0,877 \mathrm{mmol} / 1$ gemessen, der Laktatspiegel steigt bis zum Abbruch der Belastung bis 13,3 mmol/l. Nachbelastungswerte sind nicht erhoben worden, so dass keine Steigung nach Erreichen des Maximums berechnet werden kann. Der stärkste Anstieg im Verlauf der Kurve liegt zwischen den Belastungsstufen 90 und 120 Watt. 


\subsubsection{Sportler 13}

\subsection{Rennen}

Tabelle 20: Sportler 13 - Rennen Jahre 1 bis 3

\begin{tabular}{|c|c|c|c|c|c|}
\hline \multirow{2}{*}{\multicolumn{6}{|c|}{$\frac{\text { Sportler } 13}{\text { Rennen RS Jahr } 1}$}} \\
\hline & & & & & \\
\hline Rennen RS Jahr 2 & 3,2 & 2,3 & 3,3 & 3,7 & \\
\hline \multicolumn{6}{|l|}{ Rennen RS Jahr 3 (I.) } \\
\hline \multicolumn{6}{|l|}{ Rennen RS Jahr 3 (II.) } \\
\hline Mittelwert zum Messzeitpunkt & 3,2 & 2,3 & 3,3 & 3,7 & \\
\hline
\end{tabular}

\subsection{Training}

Tabelle 21: Sportler 13 - Training Jahr 1 und 2

\begin{tabular}{lllll} 
Sportler 13 & To & T1 & T2 & T3 \\
\hline Training Jahr 1 & & & & \\
\hline Training Jahr 2 & 2,4 & 2,1 & 3,2 & 3,9 \\
\hline Mittelwert zum Messzeitpunkt & 2,4 & 2,1 & 3,2 & 3,9 \\
\hline Standardabweichung & & &
\end{tabular}

Standardabweichung

Sportler 13 zeigt die maximale Laktatkonzentration mit 3,7 mmol/l zum Zeitpunkt R3, ein Abfallen der Konzentration ist innerhalb des Messzeitraums nicht zu beobachten.

Im Training wird maximal 3,9 $\mathrm{mmol} / \mathrm{l}$ bei T3 erreicht.

\subsection{Vorbereitungsphase Sommer}

Tabelle 22: Sportler 13 - Messergebnisse Sommer

\begin{tabular}{lllll} 
Sportler 13 & S0 & S1 & S2 & S3 \\
\hline Sommer 1 & 0,98 & 10,68 & 10,71 & 11,22 \\
\hline Sommer 2 & 1,5 & 10,6 & 11,1 & 11,6 \\
\hline Sommer 3 & & & & \\
\hline & & & & 11,41 \\
\hline Mittelwert zum Messzeitpunkt & 1,24 & 10,64 & 10,91 & 0,27
\end{tabular}

Die Ruhewerte der Laktatkonzentration liegen im Mittel bei 1,24 mmol/l. In der Phase nach Belastungsende steigt die Konzentration in beiden Jahren bis zum Zeitpunkt S3 an; eine Steigung nach Erreichen des Maximums ist somit nicht $\mathrm{zu}$ berechnen. Die Laktatkonzentrationen, die während der Sommeruntersuchung gemessen wurden, sind deutlich höher als jene im Wettkampf erhobenen. 


\subsubsection{Sportler 14}

\subsection{Rennen}

Tabelle 23: Sportler 14 - Rennen Jahre 1 bis 3

\begin{tabular}{llllll} 
Sportler 14 & R0 & R1 & R2 & R3 & R4 \\
\hline Rennen RS Jahr 1 & \multicolumn{7}{l}{} & & & \\
\hline Rennen RS Jahr 2 & 1,7 & 6,9 & 6,6 & 5,2 & 4,3 \\
\hline Rennen RS Jahr 3 (I.) & 1,7 & 3,8 & 4,0 & 3,9 & 3,0 \\
\hline Rennen RS Jahr 3 (II.) & & & & & \\
& & 5,35 & 5,30 & 4,55 & 3,65 \\
Mittelwert zum Messzeitpunkt & 1,70 & 2,19 & 1,84 & 0,92 & 0,92 \\
\hline Standardabweichung & 0,00 & & & &
\end{tabular}

Insgesamt liegt der Mittelwert der Ruhelaktatkonzentration bei 1,7 mmol/l. Im Mittel steigt die Konzentration bis zum Zeitpunkt R1 um 3,65 mmol/l an, das Maximum wird in der ersten Messreihe bei R1, in der zweiten Messreihe bei R2 erreicht. In beiden Messungen ist ein Absinken des Laktatspiegels innerhalb des Messzeitraums zu beobachten.

\subsection{Vorbereitungsphase Sommer}

Tabelle 24: Sportler 14 - Messergebnisse Sommer

\begin{tabular}{lllll} 
Sportler 14 & S0 & S1 & S2 & S3 \\
\hline Sommer 1 & & & & \\
\hline Sommer 2 & 2,2 & 8,8 & 10,5 & 9,2 \\
\hline Sommer 3 & & & & \\
\hline & 2,20 & 8,8 & 10,5 & 9,20 \\
\hline Mittelwert zum Messzeitpunkt & & & &
\end{tabular}

Der Ruhewert der Laktatkonzentration in der Sommermessung liegt bei 2,2 mmol/l, nach Belastung wird ein maximaler Wert von 10,5 mmol/l zum Zeitpunkt S2 erreicht, anschließend fällt die Kurve allmählich ab.

Die Laktatkonzentrationen übersteigen das Niveau der im Winter erhobenen Werte. 


\subsubsection{Stehende Sportler}

\subsubsection{1 Überblick der Wettkampfmessergebnisse Jahr 1 bis 3}

In der folgenden Abbildung sind die mittleren Konzentrationsverläufe des Laktats im Blut aller Wettkampfmessreihen der Gruppe Stehend in der Nachbelastungsphase dargestellt. Es zeigen sich große Unterschiede bei Betrachtung der Kurven sowohl im Hinblick auf die Höhe der Laktatkonzentration als auch den Verlauf der Konzentration nach Ende des Rennens.

Wie bereits bei den sitzenden Sportlern folgt zunächst eine Darstellung der einzelnen Messreihen bei den stehenden Sportlern.

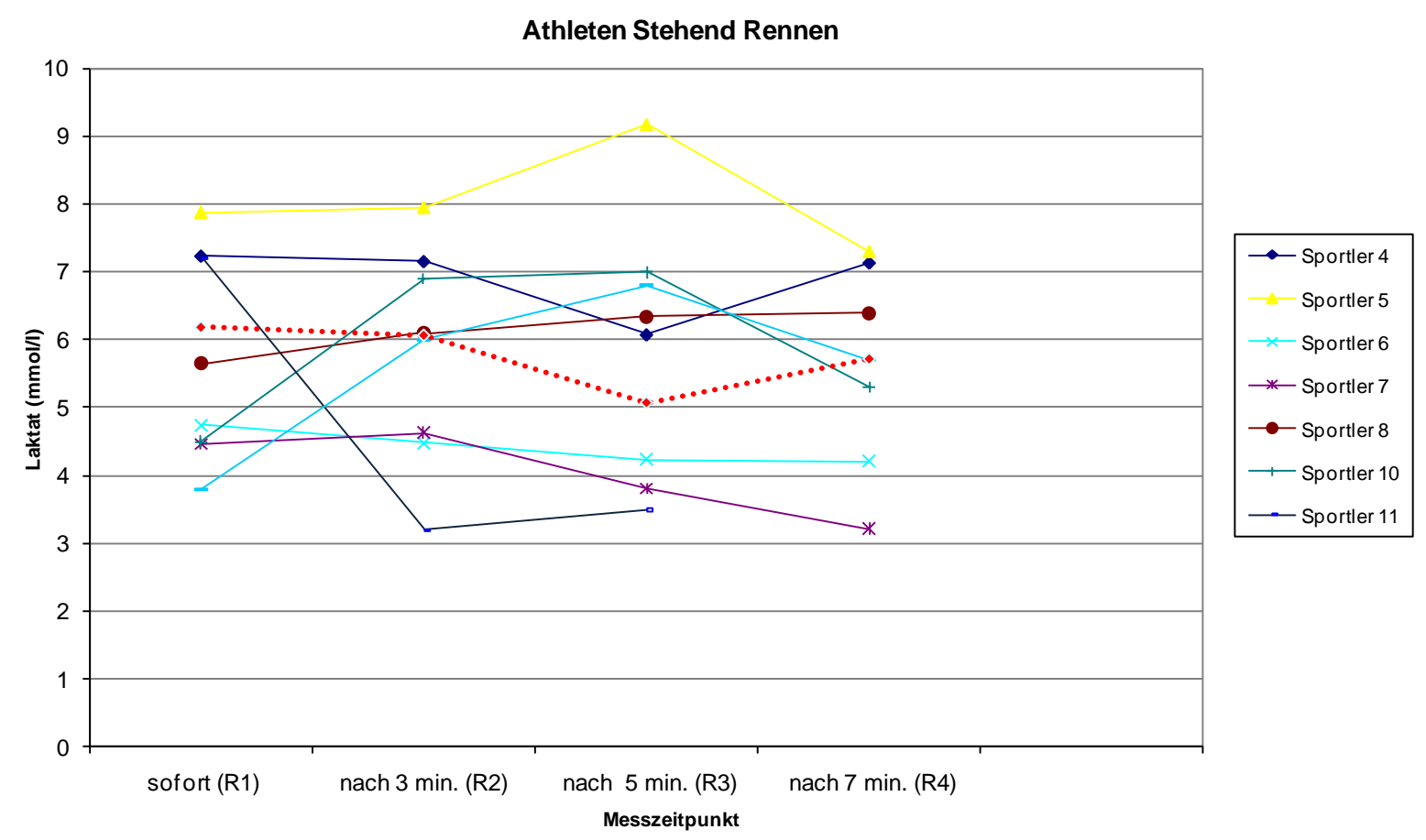

Abbildung 15: Athleten Stehend Rennen gesamt 


\subsection{Jahr 1}

Tabelle 25: Rennen Jahr 1 - Stehende Sportler

Laktat $(\mathrm{mmol} / \mathrm{l})$

Sportler Jahr 1 Stehend $\quad$ Ruhe (R0) $\quad$ sofort (R1) nach 3 min. (R2) $\quad$ nach 5 min. (R3) $\quad$ nach 7 min. (R4)

\begin{tabular}{llllll}
\hline Sportler 4 & 3,0 & 6,1 & 7,2 & 7,3 & 7,7 \\
Sportler 5 & 2,6 & 5,2 & 8,2 & 10,7 & 7,5 \\
Sportler 6 & 1,8 & 3,8 & 3,7 & 3,8 & \\
Sportler 7 & 1,6 & 4,3 & 4,5 & 4,4 & 3,5 \\
Sportler 8 & 2,1 & 6,8 & 5,1 & 6,1 & 6,4 \\
Sportler 10 & 2,7 & 4,5 & 6,9 & 7,0 & 5,3 \\
Sportler 12 & 2,5 & 3,8 & 6,0 & 6,8 & 5,7
\end{tabular}

Athleten Rennen Jahr 1 Stehend

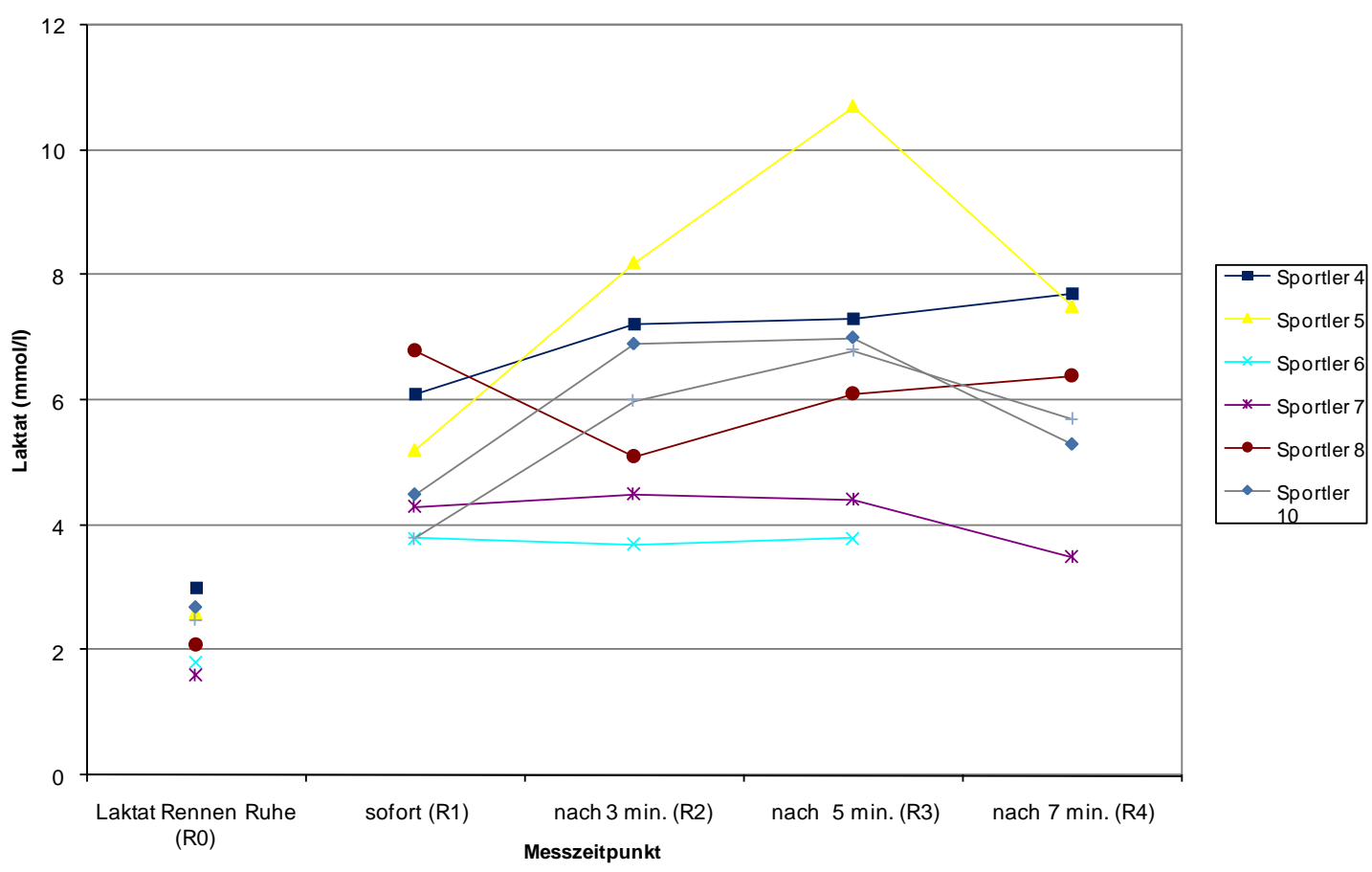

Abbildung 16: Rennen Jahr 1 - Stehende Sportler

\subsection{Jahr 2}

Tabelle 26: Rennen Jahr 2 - Stehende Sportler

Laktat $(\mathrm{mmol} / \mathrm{l})$

\begin{tabular}{lccccc} 
Sportler Jahr 2 Stehend & Ruhe (R0) & sofort (R1) & nach 3 min. (R2) & nach 5 min. (R3) & nach 7 min. (R4) \\
\hline Sportler 4 & 2,2 & 3,1 & 5,6 & 3,3 \\
Sportler 5 & 3,3 & 7,8 & 7,3 & 8,7 \\
Sportler 6 & 2,0 & 3,6 & 3,1 & 3,1 \\
Sportler 7 & 2,1 & 5,1 & 4,5 & 3,3 \\
Sportler 8 & 2,0 & 4,5 & 7,1 & 6,6 \\
Sportler 10 & 1,7 & Disqu. & & \\
Sportler 11 & 2,4 & 7,2 & 3,2 & 3,5
\end{tabular}




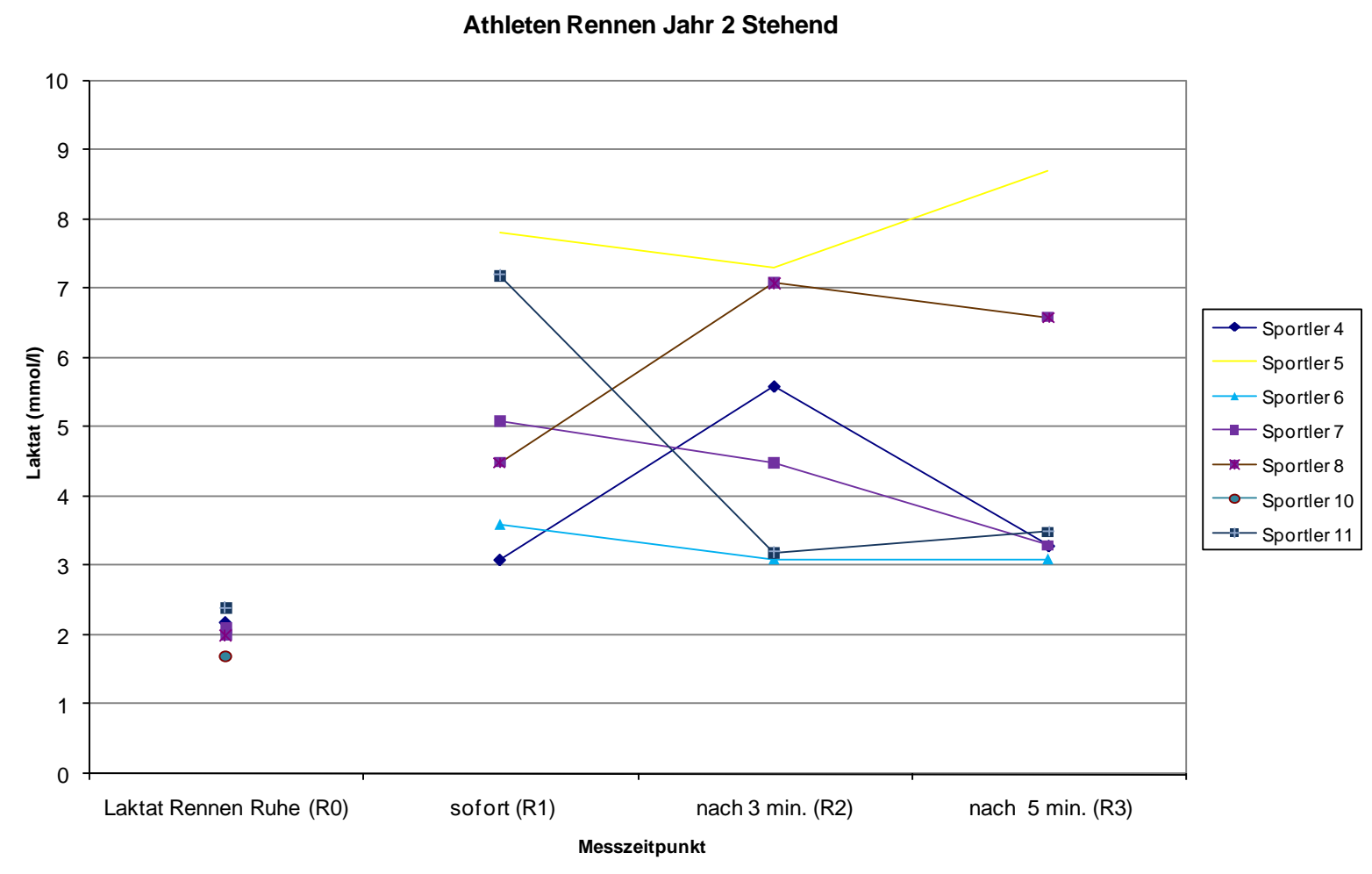

Abbildung 17: Rennen Jahr 2 - Stehende Sportler

\subsection{Jahr 3 (I.)}

Tabelle 27: Rennen Jahr 3 (I.) - Stehende Sportler

Laktat (mmol/l)

\begin{tabular}{lccccc} 
Sportler Jahr 3 (I.) Stehend & Ruhe (R0) & sofort (R1) & nach 3 min. (R2) & nach 5 min. (R3) & nach 7 min. (R4) \\
\hline Sportler 4 & 1,5 & 9,5 & 8,2 & 6,9 & 7,0 \\
Sportler 5 & 1,7 & 10,4 & 9,2 & 9,8 & 9,6 \\
Sportler 6 & 1,7 & 5,4 & 5,4 & 5,1 & 4,6 \\
Sportler 7 & 1,4 & 3,8 & 4,7 & 4,7 & 3,6
\end{tabular}




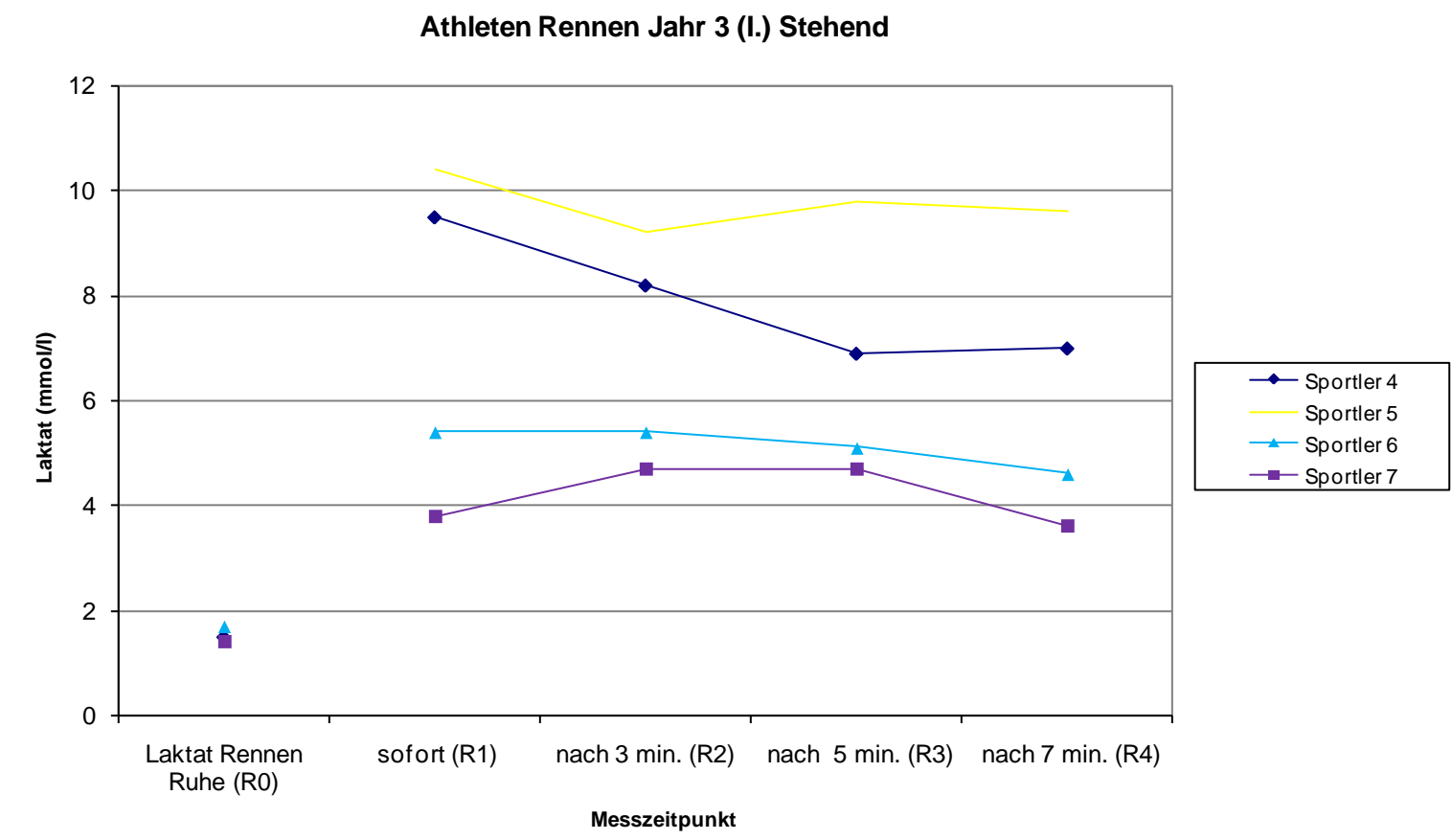

Abbildung 18: Rennen Jahr 3 (I.) - Stehende Sportler

\subsection{Jahr 3 (II.)}

Tabelle 28: Rennen Jahr 3 (II.) - Stehende Sportler

Laktat $(\mathrm{mmol} / \mathrm{l})$

Sportler Jahr 3 (II.) Stehend $\quad$ Ruhe (R0) $\quad$ sofort (R1) nach 3 min. (R2) nach 5 min. (R3) nach 7 min. (R4)

Sportler 4

2,0
10,2

7,5

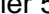

2,0

8,1

1,9

6,2

5,7

Sportler 7

1,6

$4,6 \quad 4,8$

2,8

6,6

3,8

2,5 


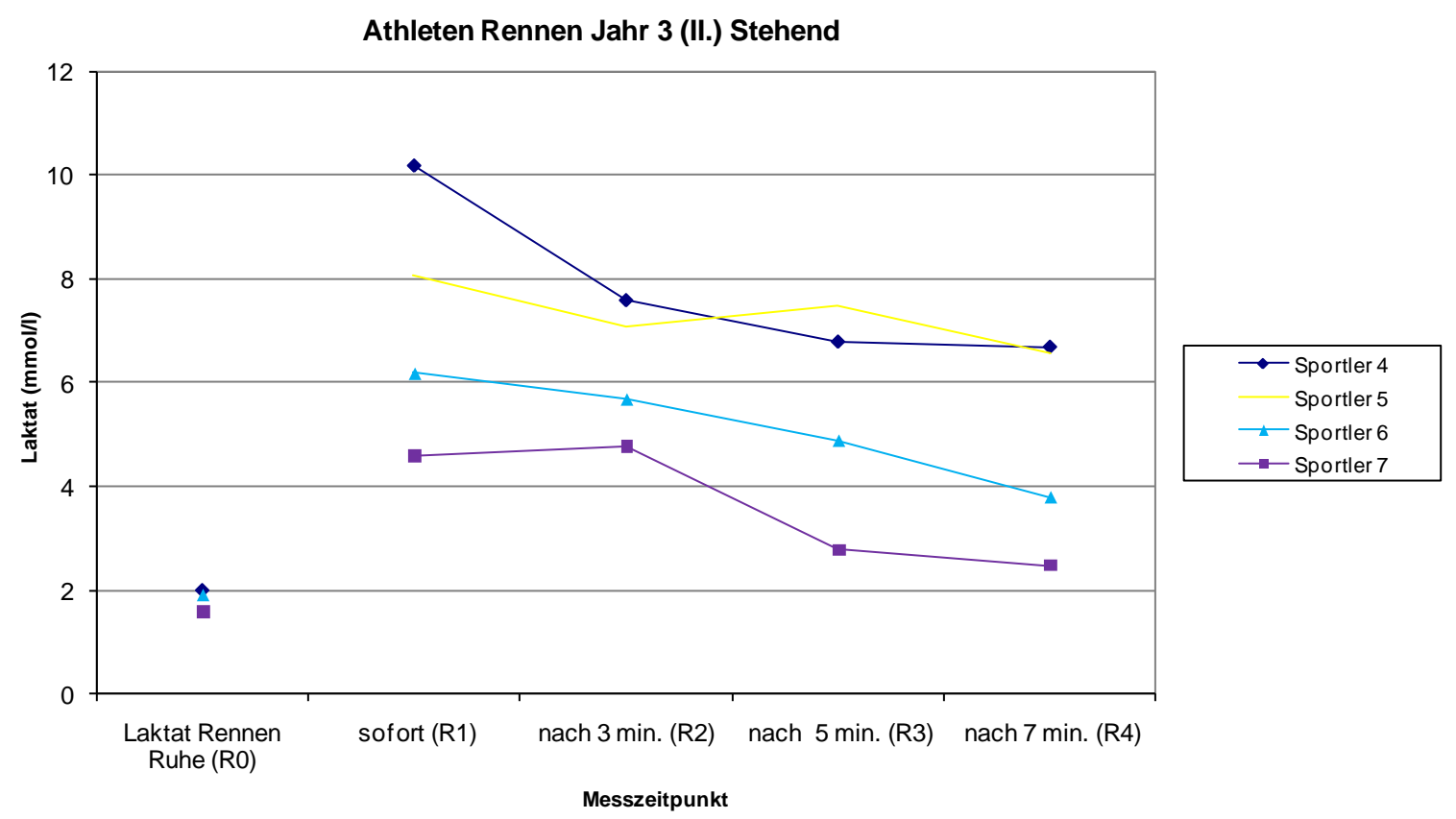

Abbildung 19: Rennen Jahr 3 (II.) - Stehende Sportler

Die Ruhewerte der stehenden Sportler im Rennen in Jahr 1 liegen im Mittel bei 2,329 mmol/l; maximal erreicht werden Laktatwerte von 3,8 bis $10,7 \mathrm{mmol} / \mathrm{l}$.

In Jahr 2 liegen die Ruhewerte der Laktatkonzentration im Kollektiv der stehenden Sportler zwischen 1,7 und 3,3 mmol/l (Mittelwert 2,24 mmol/l), die Maximalwerte der Laktatkonzentrationen betragen 3,6 bis $8,7 \mathrm{mmol} / 1$.

Im dritten Jahr der Messungen sind die Ruhewerte der Laktatkonzentrationen in der ersten Messreihe zwischen 1,4 und 1,7 mmol/l anzusiedeln, maximal erreichen die stehenden Probanden Werte von 4,7 bis 10,4 mmol/l. In der zweiten Messreihe beträgt der Mittelwert der in Ruhe gemessenen Laktatwerte 1,875 mmol/l, die Maximalwerte liegen zwischen 4,8 und $10,2 \mathrm{mmol} / \mathrm{l}$.

Die Streuung der Messergebnisse um den Mittelwert der Gruppe ist bedeutend größer als in der Gruppe Sitzend.

In der Vorbereitungsphase im Sommer wurden bei den stehenden Sportlern leistungsdiagnostische Untersuchungen mit Hilfe eines Fahrradergometers durchgeführt. Die erste Belastungsstufe und die Steigerung der Belastung variierten innerhalb der Probandengruppe. 


\subsubsection{Sportler 4}

\subsection{Rennen}

Tabelle 29: Sportler 4 - Rennen Jahre 1 bis 3

\begin{tabular}{|c|c|c|c|c|c|}
\hline Sportler 4 & $\begin{array}{l}\text { Laktat Rennen } \\
\text { Ruhe (R0) }\end{array}$ & sofort (R1) & nach 3 min. (R2) & nach 5 min. (R3) & nach $7 \mathrm{~min} .(\mathrm{R} 4)$ \\
\hline Rennen RS Jahr 1 & 3,0 & 6,1 & 7,2 & 7,3 & 7,7 \\
\hline Rennen RS Jahr 2 & 2,2 & 3,1 & 5,6 & 3,3 & \\
\hline Rennen RS Jahr 3 (I.) & 1,5 & 9,5 & 8,2 & 6,9 & 7,0 \\
\hline Rennen RS Jahr 3 (II.) & 2,0 & 10,2 & 7,6 & 6,8 & 6,7 \\
\hline Mittelwert zum Messzeitpunkt & 2,18 & 7,23 & 7,15 & 6,08 & 7,13 \\
\hline Standardabweichung & 0,62 & 3,28 & 1,11 & 1,86 & 0,51 \\
\hline
\end{tabular}

\subsection{Training}

Tabelle 30: Sportler 4 - Training Jahr 1 und 2

\begin{tabular}{lllll}
\multicolumn{2}{l}{$\begin{array}{l}\text { Laktat Training } \\
\text { Spuhe (T0) }\end{array}$} & sofort (T1) & nach 3 min. (T2) & nach 5 min. (T3) \\
\hline Training Jahr 1 & 2,0 & 3,1 & 3,9 & 3,0 \\
\hline Training Jahr 2 & 1,6 & 3,1 & 3,7 & 2,2 \\
\hline Mittelwert zum Messzeitpunkt & 1,8 & 3,1 & 3,8 & 2,6 \\
\hline Standardabweichung & 0,28 & 0,00 & 0,14 & 0,57
\end{tabular}

Insgesamt schwanken die während des Wettkampfes gemessenen Laktatkonzentrationen im Blut in Ruhe - also zum Zeitpunkt R0 - stark zwischen 1,5 und 3,0 mmol/l; im Mittel beträgt die Konzentration bei R0 2,18 mmol/l. Zwischen R0 und R1 steigt der Wert in allen Messreihen um im Mittel 5,05 mmol/l an. Maximalwerte werden zu unterschiedlichen Zeitpunkten erreicht, eine Steigung der Laktatverlaufskurve ist nicht immer anzugeben, in drei Messreihen ist sie nach Erreichen des Maximums negativ.

Im Training variieren die Laktatkonzentrationen in Ruhe, im Mittel sind sie bei $1,8 \mathrm{mmol} / \mathrm{l}$ einzuordnen. Die maximale Laktatkonzentration erreicht im Training Werte unter $4 \mathrm{mmol} / \mathrm{l}$. Im Rennen $\mathrm{zu}$ verzeichnende Maximalwerte werden im Training nicht erreicht. Die Laktatkonzentration nimmt in beiden Jahren bis zum Zeitpunkt $\mathrm{T} 2 \mathrm{zu}$, anschließend fällt die Kurve ab.

\subsection{Vorbereitungsphase Sommer}

Tabelle 31: Sportler 4 - Messergebnisse Sommer

\begin{tabular}{|c|c|c|c|c|c|c|c|c|c|c|c|}
\hline Sportler 4 & Ruhe (S0) & $75 \mathrm{~W}$ & $125 \mathrm{~W}$ & $175 \mathrm{~W}$ & $225 \mathrm{~W}$ & $275 \mathrm{~W}$ & $325 \mathrm{~W}$ & $375 \mathrm{~W}$ & $425 \mathrm{~W}$ & sofort (S1) & $\mathrm{S} 2$ \\
\hline Sommer 1 & 1,17 & 1,32 & 1,6 & 2,01 & 2,54 & 3,24 & 4,85 & 6,97 & 11,2 & 12,9 & \\
\hline Sommer 2 & 2,0 & 1,7 & 1,7 & 2,2 & 7,3 & 3,8 & 4,9 & 8,9 & 13,3 & 13,8 & 13,3 \\
\hline \multicolumn{12}{|l|}{ Sommer 3} \\
\hline Mittelwert z. Messzeitp. & 1,59 & 1,51 & 1,65 & 2,11 & 4,92 & 3,52 & 4,88 & 7,935 & 12,25 & 13,35 & 13,3 \\
\hline Standardabweichung & 0,59 & 0,27 & 0,07 & 0,13 & 3,37 & 0,40 & 0,04 & 1,36 & 1,48 & 0,64 & \\
\hline
\end{tabular}


Die leistungsdiagnostische Untersuchung von Sportler 4 im Sommer beginnt mit einer Belastungsstufe von 75 Watt und wird um jeweils 50 Watt gesteigert. Maximal erreicht Sportler 4425 Watt, die gemessenen Laktatwerte zu diesem Zeitpunkt liegen im Mittel bei $12,25 \mathrm{mmol} / \mathrm{l}$. Zwischen den Belastungsstufen steigt die Konzentration zunächst um Differenzen von kleiner $1 \mathrm{mmol} / \mathrm{l}$ an, stärkere Anstiege sind ab einer Belastung von 325 Watt zu verzeichnen. Zwischen 375 Watt und 425 Watt steigt die Kurve dann um 4,4 mmol/l an. Nach Erreichen der Belastungsstufe von 425 Watt wird die Belastung abgebrochen. Im ersten Jahr wurde ein Nachbelastungswert unmittelbar nach Abbruch der Belastung bestimmt, die Laktatkonzentration steigt weiter an. Im zweiten Jahr steigt die Konzentration bis zum Zeitpunkt S1 an, fällt dann zum Zeitpunkt S2 leicht ab; eine Aussage zum weiteren Kurvenverlauf ist nicht möglich.

\subsubsection{Sportler 5}

\subsection{Rennen}

Tabelle 32: Sportler 5 - Rennen Jahre 1 bis 3

\begin{tabular}{llllll} 
Sportler 5 & R0 & R1 & R2 & R3 & R4 \\
\hline Rennen RS Jahr 1 & 2,6 & 5,2 & 8,2 & 10,7 & 7,5 \\
\hline Rennen RS Jahr 2 & 3,3 & 7,8 & 7,3 & 8,7 & \\
\hline Rennen RS Jahr 3 (I.) & 1,7 & 10,4 & 9,2 & 9,8 & 9,6 \\
\hline Rennen RS Jahr 3 (II.) & 2,0 & 8,1 & 7,1 & 7,5 & 6,6 \\
\hline & & & & & 7,9 \\
\hline Mittelwert zum Messzeitpunkt & 2,40 & 7,88 & 7,95 & 9,18 & 1,54 \\
\hline Standardabweichung & 0,71 & 2,13 & 0,96 & 1,38 & \\
\hline
\end{tabular}

\subsection{Training}

Tabelle 33: Sportler 5 - Training Jahr 1 und 2

\begin{tabular}{lllll} 
Sportler $\mathbf{5}$ & T0 & T1 & T2 & T3 \\
\hline Training Jahr 1 & 2,4 & 4,4 & 3,8 & 3,7 \\
\hline Training Jahr 2 & 1,7 & 4,4 & 4,0 & 3,4 \\
\hline Mittelwert zum Messzeitpunkt & 2,05 & 4,4 & 3,9 & 3,55 \\
\hline Standardabweichung & 0,49 & 0,00 & 0,14 & 0,21
\end{tabular}

Insgesamt zeigt sich in den Messungen während des Rennens eine deutliche Schwankung der Ruhelaktatwerte zum Zeitpunkt R0, sie variieren zwischen 1,7 und 3,3 mmol/l, im Mittel liegen sie bei 2,4 mmol/l. Zwischen den Zeitpunkten R0 und R1 zeigen sich stets starke Anstiege der Laktatkonzentration im Blut, die Differenz beträgt im Mittelwert 5,48 mmol/l. Maximalwerte erreicht die Laktatkonzentration in den Jahren 1 und 2 zum Zeitpunkt R3, in Jahr 3 bei R1. Die Steigung nach Erreichen des Maximums variiert deutlich; Wiederanstiege der Kurve sind zu beobachten (Rennen Jahr 3 I und II). 
Im Training variieren die Laktatwerte zum Zeitpunkt T0 ebenfalls, im Mittel liegen sie bei 2,05 mmol/1, die maximale Konzentration des Laktats im Blut beträgt in beiden Messreihen 4,4 mmol/1 zum Zeitpunkt T1; im Wettkampf beobachtete Konzentrationsniveaus werden nicht erreicht. Die Steigung nach Erreichen des Maximums ist negativ.

\subsection{Vorbereitungsphase Sommer}

Tabelle 34: Sportler 5 - Messergebnisse Sommer

\begin{tabular}{|c|c|c|c|c|c|c|c|}
\hline Sportler 5 & so & $75 \mathrm{~W}$ & $125 \mathrm{~W}$ & $175 \mathrm{~W}$ & $225 \mathrm{~W}$ (S1) & S2 & S3 \\
\hline Sommer 1 & 1,38 & 2,76 & 4,88 & 8,8 & 19,2 & & \\
\hline Sommer 2 & 3,4 & 3,0 & 4,5 & 6,4 & 18,5 & 17,6 & 15,1 \\
\hline \multicolumn{8}{|l|}{ Sommer 3} \\
\hline Mittelwert zum Messzeitpunkt & 2,39 & 2,88 & 4,69 & 7,60 & 18,85 & 17,6 & 15,10 \\
\hline Standardabweichung & 1,43 & 0,17 & 0,27 & 1,70 & 0,49 & & \\
\hline
\end{tabular}

Sportler 5 beginnt die Untersuchung mit einer Belastung von 75 Watt, eine Steigerung der Belastung erfolgt in 50-Watt-Schritten. In Sommer 1 zeigt sich zunächst ein Anstieg der Laktatkonzentration um $1,38 \mathrm{mmol} / \mathrm{l}$; die Konzentration nimmt insgesamt deutlich $\mathrm{zu}$; maximal zwischen der Messung des Werts nach der Stufe 175 Watt und S1. In der Nachbelastungsphase sind keine Werte erhoben worden. In Jahr 2 fällt die Konzentration vom Zeitpunkt S0 zunächst leicht ab und steigt anschließend in Schritten bis 1,9 mmol/l an; am Ende der Belastung, also zwischen der Messung nach 175 Watt und S1, nimmt die Konzentration um 12,1 mmol/l zu. In der Nachbelastungsphase fällt die Kurve leicht ab. Die in den Sommermessungen erreichten Maximalwerte übersteigen jene während des Winters in Wettkampf und Training erhobenen Maximalwerte.

\subsubsection{Sportler 6}

\subsection{Rennen}

Tabelle 35: Sportler 6 - Rennen Jahre 1 bis 3

\begin{tabular}{llllll} 
Sportler 6 & $\mathrm{R} 0$ & $\mathrm{R} 1$ & $\mathrm{R} 2$ & $\mathrm{R} 3$ & $\mathrm{R} 4$ \\
\hline Rennen RS Jahr 1 & 1,8 & 3,8 & 3,7 & 3,8 & \\
\hline Rennen RS Jahr 2 & 2,0 & 3,6 & 3,1 & 3,1 & 4,6 \\
\hline Rennen RS Jahr 3 (I.) & 1,7 & 5,4 & 5,4 & 5,1 & 3,8 \\
\hline Rennen RS Jahr 3 (II.) & 1,9 & 6,2 & 5,7 & 4,9 & \\
\hline & & & & & 4,20 \\
\hline Mittelwert zum Messzeitpunkt & 1,85 & 4,75 & 4,48 & 0,94 & 0,57 \\
\hline Standardabweichung & 0,13 & 1,26 & 1,27 & &
\end{tabular}




\subsection{Training}

Tabelle 36: Sportler 6 - Training Jahr 1 und 2

\begin{tabular}{lcccc} 
Sportler 6 & T0 & T1 & T2 & T3 \\
\hline Training Jahr 1 & & & & 3,4 \\
\hline Training Jahr 2 & 1,8 & 5,5 & 4,3 & 3,4 \\
\hline Mittelwert zum Messzeitpunkt & 1,8 & 5,5 & 4,3 &
\end{tabular}

Insgesamt liegen die Laktatwerte zum Zeitpunkt R0 bei $2 \mathrm{mmol} / \mathrm{l}$ Blut, im Mittel beträgt der Ruhewert 1,85 mmol/l. Der größte Anstieg der Konzentration zeigt sich zwischen R0 und R1 mit durchschnittlich 4,75 mmol/l. Sportler 6 erreicht in vier Messreihen die maximale Laktatkonzentration zum Zeitpunkt R1, in drei Messungen ist danach ein Abfall des Laktatspiegels nachzuweisen. Der Maximalwert bleibt stets unterhalb einer Konzentration von $6,3 \mathrm{mmol} / 1$.

Im Training wird eine maximale Laktatkonzentration von $5,5 \mathrm{mmol} / \mathrm{l}$ erreicht (T1). Anschließend ist ein leichter Rückgang zu beobachten.

\subsection{Vorbereitungsphase Sommer}

Tabelle 37: Sportler 6 - Messergebnisse Sommer

\begin{tabular}{|c|c|c|c|c|c|c|c|c|c|}
\hline Sportler 6 & So & $75 \mathrm{~W}$ & $100 \mathrm{~W}$ & $125 \mathrm{~W}$ & $150 \mathrm{~W}$ & $175 \mathrm{~W}$ & S1 & $\mathrm{S} 2$ & S3 \\
\hline Sommer 1 & 0,74 & 1,79 & 2,64 & 4,59 & 7,86 & & & & \\
\hline \multicolumn{10}{|l|}{ Sommer 2} \\
\hline Sommer 3 & 1,6 & 3,6 & 3,7 & 4,7 & 6,2 & 7,8 & 8,7 & 6,7 & 7,3 \\
\hline Mittelwert zum Messzeitpunkt & 1,17 & 2,70 & 3,17 & 4,65 & 7,03 & 7,8 & 8,70 & 6,7 & 7,3 \\
\hline Standardabweichung & 0,61 & 1,28 & 0,75 & 0,08 & 1,17 & & & & \\
\hline
\end{tabular}

Die Belastungsstufe zu Beginn der Untersuchung im Sommer wird bei Sportler 6 bei 75 Watt festgesetzt, die Steigerung der Belastung erfolgt in 25-Watt-Schritten. Die Laktatkonzentration in Ruhe variiert zwischen den Jahren 1 und 3, im Mittel beträgt sie 1,17 mmol/l. Durchschnittlich steigt die Konzentration des Laktats innerhalb der Belastungsstufen um maximal 3,27 mmol/l an, in beiden Untersuchungen wird die Belastung auf der Stufe von 175 Watt abgebrochen. In Jahr 1 sind keine Werte nach Belastung bestimmt worden, in Jahr 3 zeigt sich ein Anstieg bis zu einer maximalen Konzentration von 8,7 mmol Laktat/l Blut zum Zeitpunkt S1. Anschließend ist die Laktatkurve rückläufig. Der nach fünf Minuten gemessene Wert (S3) liegt bereits wieder unterhalb des Wertes, der bei Abbruch der Belastung bestimmt worden ist. 


\subsubsection{Sportler 7}

\subsection{Rennen}

Tabelle 38: Sportler 7 - Rennen Jahre 1 bis 3

\begin{tabular}{llllll} 
Sportler 7 & R0 & R1 & R2 & R3 & R4 \\
\hline Rennen RS Jahr 1 & 1,6 & 4,3 & 4,5 & 4,4 & 3,5 \\
\hline Rennen RS Jahr 2 & 2,1 & 5,1 & 4,5 & 3,3 & \\
\hline Rennen RS Jahr 3 (I.) & 1,4 & 3,8 & 4,7 & 4,7 & \\
\hline Rennen RS Jahr 3 (II.) & 1,6 & 4,6 & 4,8 & 2,8 & \\
\hline & & & & & 3,6 \\
\hline Mittelwert zum Messzeitpunkt & 1,68 & 4,45 & 4,63 & 0,90 & 3,2 \\
\hline Standardabweichung & 0,30 & 0,54 & 0,15 & 0,61 \\
\hline
\end{tabular}

\subsection{Training}

Tabelle 39: Sportler 7 - Training Jahr 1 und 2

\begin{tabular}{lllll} 
Sportler 7 & T0 & T1 & T2 & T3 \\
\hline Training Jahr 1 & 1,7 & 2,8 & 2,4 & 2,0 \\
\hline Training Jahr 2 & 2,2 & 4,0 & 2,2 & 1,9 \\
\hline Mittelwert zum Messzeitpunkt & 1,95 & 3,4 & 2,3 & 1,95 \\
\hline Standardabweichung & 0,35 & 0,85 & 0,14 & 0,07
\end{tabular}

Im Vergleich der vier Messreihen zeigt sich ein mittlerer R0-Wert von 1,68 mmol/l, in Jahr 2 liegt er höher als in den anderen Messungen. Der stärkste Anstieg der Laktatkonzentration ist zwischen R0 und R1 zu beobachten mit einem durchschnittlichen Wert von 2,775 mmol/l. Maximal werden Konzentrationen von 5,1 mmol Laktat/l Blut bestimmt, der Zeitpunkt des Erreichens des Maximums liegt in den Jahren 1 und 3 bei R2, in Jahr 2 bei R1. Anschließend fällt die Kurve ab.

Im Training streuen die Ergebnisse zum Zeitpunkt R0 deutlich, im Mittel beträgt die Ruhelaktatkonzentration 1,95 mmol/l Blut. Zum Zeitpunkt T1 wird ein Maximum erreicht, innerhalb des Messzeitraums liegen die Werte bereits wieder im Ruhewertniveau.

\subsection{Vorbereitungsphase Sommer}

Tabelle 40: Sportler 7 - Messergebnisse Sommer

\begin{tabular}{|c|c|c|c|c|c|c|c|}
\hline Sportler 7 & so & $75 \mathrm{~W}$ & $100 \mathrm{~W}$ & $125 \mathrm{~W}$ & $150 \mathrm{~W}$ & 175 W (S1) & S2 \\
\hline Sommer 1 & 1,25 & 2,04 & 3,54 & 4,54 & 6,6 & 10,0 & 12,50 \\
\hline \multicolumn{8}{|l|}{ Sommer 2} \\
\hline Sommer 3 & & & & & & & \\
\hline Mittelwert zum Messzeitpunkt & 1,25 & 2,04 & 3,54 & 4,54 & 6,6 & 10,0 & 12,50 \\
\hline
\end{tabular}

Standardabweichung

Bei Sportler 7 beträgt die erste Belastungsstufe in den Sommeruntersuchungen 75 Watt, die Steigerung erfolgt in 25-Watt-Schritten. Sportler 7 erreicht die Belastungsstufe von 175 Watt, zwischen den jeweiligen Stufen steigt die Laktatkonzentration im Blut in Abständen von 
maximal $3,4 \mathrm{mmol} / \mathrm{l}$ bis zu einem Wert von $10,0 \mathrm{mmol} / \mathrm{l}$ bei Belastungsende an. In der Nachbelastungsphase wird ein Maximum von 12,5 mmol/l erreicht, das deutlich über den maximal erreichten Konzentrationswerten in Rennen und Training im Winter liegt. Eine Steigung nach dem Maximum ist nicht zu errechnen, da keine weiteren Nachbelastungswerte erhoben wurden.

\subsubsection{Sportler 8}

\subsection{Rennen}

Tabelle 41: Sportler 8 - Rennen Jahre 1 bis 3

\begin{tabular}{llllll} 
Sportler 8 & R0 & R1 & R2 & R3 & R4 \\
\hline Rennen RS Jahr 1 & 2,1 & 6,8 & 5,1 & 6,1 & 6,4 \\
\hline Rennen RS Jahr 2 & 2,0 & 4,5 & 7,1 & 6,6 \\
\hline Rennen RS Jahr 3 (I.) & & & & & \\
\hline Rennen RS Jahr 3 (II.) & & & & 6,35 & 6,40 \\
\hline & & 5,65 & 6,10 & 0,35 & \\
\hline Mittelwert zum Messzeitpunkt & 2,05 & 1,63 & 1,41 & \\
\hline Standardabweichung & 0,07 & &
\end{tabular}

\subsection{Training}

Tabelle 42: Sportler 8 - Training Jahr 1 und 2

\begin{tabular}{lcccc} 
Sportler 8 & T0 & T1 & T2 & T3 \\
\hline Training Jahr 1 & & & & 3,2 \\
\hline Training Jahr 2 & 1,5 & 3,3 & 3,9 & 3,2 \\
\hline Mittelwert zum Messzeitpunkt & 1,5 & 3,3 & 3,9 &
\end{tabular}

Insgesamt liegt der Laktatwert zum Zeitpunkt R0 bei Sportler 8 in den Jahren 1 und 2 bei 2,05 mmol/l Blut, der stärkste Anstieg der Verlaufskurve der Laktatkonzentration ist zwischen den Zeitpunkten R0 und R1 zu verzeichnen, mit im Mittel 3,6 mmol/l. Die Kurven der Jahre 1 und 2 unterscheiden sich: In Jahr 1 wird das Maximum zum Zeitpunkt R1 erreicht, in Jahr 2 bei R2. Im ersten Jahr steigt die Kurve nach dem Maximum erneut an.

Im Training lassen sich keine Vergleiche der unterschiedlichen Jahre anstellen, eine maximale Konzentration wird bei T2 erreicht, anschließend zeigt sich ein leichter Rückgang. 


\subsection{Vorbereitungsphase Sommer}

Tabelle 43: Sportler 8 - Messergebnisse Sommer

\begin{tabular}{|c|c|c|c|c|c|c|c|c|}
\hline Sportler 8 & So & $75 \mathrm{~W}$ & $125 \mathrm{~W}$ & $175 \mathrm{~W}$ & $225 \mathrm{~W}$ & $275 \mathrm{~W}(\mathrm{~S} 1)$ & S2 & S3 \\
\hline \multicolumn{9}{|l|}{ Sommer 1} \\
\hline Sommer 2 & 1,9 & 2,0 & 1,7 & 2,4 & 3,6 & 6,6 & 6,5 & 7,1 \\
\hline \multicolumn{9}{|l|}{ Sommer 3} \\
\hline Mittelwert zum Messzeitpunkt & 1,90 & 2,0 & 1,70 & 2,40 & 3,60 & 6,60 & 6,50 & 7,10 \\
\hline
\end{tabular}

Auch Proband 8 beginnt in der leistungsdiagnostischen Untersuchung im Sommer mit einer Belastung von 75 Watt, die Steigerung der Belastung erfolgt in 50-Watt-Schritten. Die Laktatkonzentration in Ruhe beträgt $1,9 \mathrm{mmol} / \mathrm{l}$ und steigt bis zur 225-Watt-Stufe in Abständen von bis zu 1,2 mmol/1 an; bei 275 Watt wird die Belastung abgebrochen, der Laktatwert liegt zu diesem Zeitpunkt $\mathrm{S} 1$ bei $6,6 \mathrm{mmol} / 1$ und erreicht bei $\mathrm{S} 3$ seinen Maximalwert.

Die in der Vorbereitungsphase erhobenen Werte liegen im Bereich der während des Rennens im Winter gemessenen.

\subsubsection{Sportler 10}

\subsection{Rennen}

Tabelle 44: Sportler 10 - Rennen Jahre 1 bis 3

\begin{tabular}{llllll} 
Sportler 10 & R0 & R1 & R2 & R3 & R4 \\
\hline Rennen RS Jahr 1 & 2,7 & 4,5 & 6,9 & 7 & 5,3 \\
\hline Rennen RS Jahr 2 & 1,7 & Disqualifikation & & & \\
\hline Rennen RS Jahr 3 (I.) & & & & & \\
\hline Rennen RS Jahr 3 (II.) & & & & & \\
\hline & & & & & \\
\hline Mittelwert zum Messzeitpunkt & 2,2 & 4,5 & & & \\
\hline Standardabweichung & 0,71 & & & & \\
\hline
\end{tabular}

\subsection{Training}

Tabelle 45: Sportler 10 - Training Jahr 1 und 2

\begin{tabular}{lllll} 
Sportler 10 & T0 & T1 & T2 & T3 \\
\hline Training Jahr 1 & & & & \\
\hline Training Jahr 2 & 1,9 & 1,7 & 2,7 & 1,7 \\
\hline Mittelwert zum Messzeitpunkt & 1,90 & 1,70 & 2,70 & 1,70 \\
\hline Standardabweichung & & & &
\end{tabular}

Die Ruhewerte im Rennen liegen im Mittel bei 2,2 mmol/l. Auf Grund einer Disqualifikation ist ein weiterer Vergleich nicht möglich. In Jahr 1 erreicht Sportler 10 eine maximale Laktatkonzentration bei R3, anschließend fällt sie leicht ab.

Im Training liegt das Maximum bei $2,7 \mathrm{mmol} / \mathrm{l}$ zum Zeitpunkt $\mathrm{T} 2$ und fällt auf Ruhewertniveau ab. 


\subsection{Vorbereitungsphase Sommer}

Tabelle 46: Sportler 10 - Messergebnisse Sommer

\begin{tabular}{|c|c|c|c|c|c|c|}
\hline Sportler 10 & so & $75 \mathrm{~W}$ & $125 \mathrm{~W}$ & $175 \mathrm{~W}$ & 225 W (S1) & S2 \\
\hline Sommer 1 & 1,25 & 1,62 & 4,12 & 5,64 & 11,5 & 15,8 \\
\hline Sommer 2 & & & & & & \\
\hline Sommer 3 & & & & & & \\
\hline Mittelwert zum Messzeitpunkt & 1,25 & 1,62 & 4,12 & 5,64 & 11,5 & 15,8 \\
\hline
\end{tabular}

Der Ruhelaktatwert im Blut während der Untersuchungen in der Vorbereitungsphase im Sommer liegt bei Sportler 10 bei 1,25 mmol/l, die Belastung beginnt bei 75 Watt und wird in 50-Watt-Abständen gesteigert. Die Laktatkonzentration nimmt zwischen den Belastungsstufen deutlich zu. Die Leistung wird bei 225 Watt abgebrochen; zu diesem Zeitpunkt beträgt der Laktatwert 11,5 mmol/l und steigt in der Phase nach Belastungsende bis zu einem Maximum von 15,8 mmol/l an. Die Konzentrationen des Laktats im Blut sind höher als die in Rennen und Training im Winter gemessenen Werte.

\subsubsection{Sportler 11}

\subsection{Rennen}

Tabelle 47: Sportler 11 - Rennen Jahre 1 bis 3

\begin{tabular}{|c|c|c|c|c|c|}
\hline Sportler 11 & Ro & $\mathrm{R} 1$ & $\mathrm{R} 2$ & R3 & $\mathrm{R} 4$ \\
\hline \multicolumn{6}{|l|}{ Rennen RS Jahr 1} \\
\hline Rennen RS Jahr 2 & 2,4 & 7,2 & 3,2 & 3,5 & \\
\hline \multicolumn{6}{|l|}{ Rennen RS Jahr 3 (I.) } \\
\hline \multicolumn{6}{|l|}{ Rennen RS Jahr 3 (II.) } \\
\hline Mittelwert zum Messzeitpunkt & 2,40 & 7,20 & 3,20 & 3,50 & \\
\hline
\end{tabular}

\subsection{Training}

Tabelle 48: Sportler 11 - Training Jahr 1 und 2

\begin{tabular}{lllll} 
Sportler 11 & T0 & T1 & T2 & T3 \\
\hline Training Jahr 1 & 2,2 & 5,1 & 4,3 & 3,2 \\
\hline Training Jahr 2 & 3,3 & 4,6 & 3,6 & 2,3 \\
\hline & & & & 2,75 \\
\hline Mittelwert zum Messzeitpunkt & 2,75 & 4,85 & 3,95 & 0,64
\end{tabular}

Die maximale Laktatkonzentration beträgt bei R1 7,2 mmol/l Blut, anschließend fällt sie rasch ab und steigt erneut an. 
Insgesamt liegt der mittlere Ruhewert der Laktatkonzentration im Blut vor dem Trainingslauf bei 2,75 mmol/l, die Konzentration steigt stark an, im Mittel um 2,1 mmol/l bis zum Zeitpunkt T1. Die Kurve fällt in beiden Jahren nach dem Maximum allmählich ab.

\subsection{Vorbereitungsphase Sommer}

Tabelle 49: Sportler 11 - Messergebnisse Sommer

\begin{tabular}{|c|c|c|c|c|c|c|c|}
\hline Sportler 11 & So & $75 \mathrm{~W}$ & $125 \mathrm{~W}$ & $175 \mathrm{~W}$ & $225 \mathrm{~W}$ (S1) & S2 & S3 \\
\hline \multicolumn{8}{|l|}{ Sommer 1} \\
\hline Sommer 2 & 1,5 & 1,8 & 3,4 & 5,8 & 7,7 & 4,4 & 3,5 \\
\hline \multicolumn{8}{|l|}{ Sommer 3} \\
\hline Mittelwert zum Messzeitpunkt & 1,50 & 1,80 & 3,40 & 5,80 & 7,70 & 4,40 & 3,50 \\
\hline
\end{tabular}

Die erste Belastungsstufe im Sommer bei Sportler 11 beträgt 75 Watt, eine Steigerung erfolgt in 50-Watt-Schritten. Der Ruhewert liegt mit 1,5 mmol/l niedriger als in Training und Rennen im Winter, die Laktatkonzentration steigt bis zum Zeitpunkt $\mathrm{S} 1$ bis $7,7 \mathrm{mmol} / \mathrm{l}$ an. Die Belastung wird bei 225 Watt abgebrochen. In der Nachbelastungsphase ist ein Rückgang der Laktatkonzentration zu beobachten.

\subsubsection{Sportler 12}

\subsection{Rennen}

Tabelle 50: Sportler 12 - Rennen Jahre 1 bis 3

\begin{tabular}{llllll} 
Sportler 12 & R0 & R1 & R2 & R3 & R4 \\
\hline Rennen RS Jahr 1 & 2,5 & 3,8 & 6,0 & 6,8 & 5,7 \\
\hline Rennen RS Jahr 2 & & & & & \\
\hline Rennen RS Jahr 3 (I.) & & & & & \\
\hline Rennen RS Jahr 3 (II.) & & & & & \\
\hline & & & & & \\
\hline Mittelwert zum Messzeitpunkt & 2,50 & 3,80 & & & \\
\hline Standardabweichung & & & &
\end{tabular}

\subsection{Training}

Tabelle 51: Sportler 12 - Training Jahr 1 und 2

\begin{tabular}{lllll} 
Sportler $\mathbf{1 2}$ & T0 & T1 & T2 & T3 \\
\hline Training Jahr 1 & 2,4 & 4,9 & 5,2 & 3,8 \\
\hline Training Jahr 2 & & & & \\
\hline Mittelwert zum Messzeitpunkt & 2,40 & 4,90 & 5,20 & 3,80 \\
\hline Standardabweichung & &
\end{tabular}


Sportler 12 hat sowohl im Wettkampf als auch im Training ausschließlich in Jahr 1 teilgenommen. Vergleiche sind daher nicht möglich. Er erreicht maximal eine Konzentration von 6,8 mmol Laktat/l Blut (bei R3). Die Konzentration nach Belastung ist nur langsam rückläufig. Der Ruhewert wird nicht erreicht.

Im Training beträgt die Maximalkonzentration 5,2 mmol Laktat/l Blut; zum Zeitpunkt T3 ist der Laktatspiegel abgesunken, erreicht jedoch nicht Ruhewertniveau.

\subsubsection{Vergleich sitzende und stehende Sportler}

Zum Abschluss der Einzelfallbetrachtung werden erneut die Probandengruppen Sitzend und Stehend gegenübergestellt.

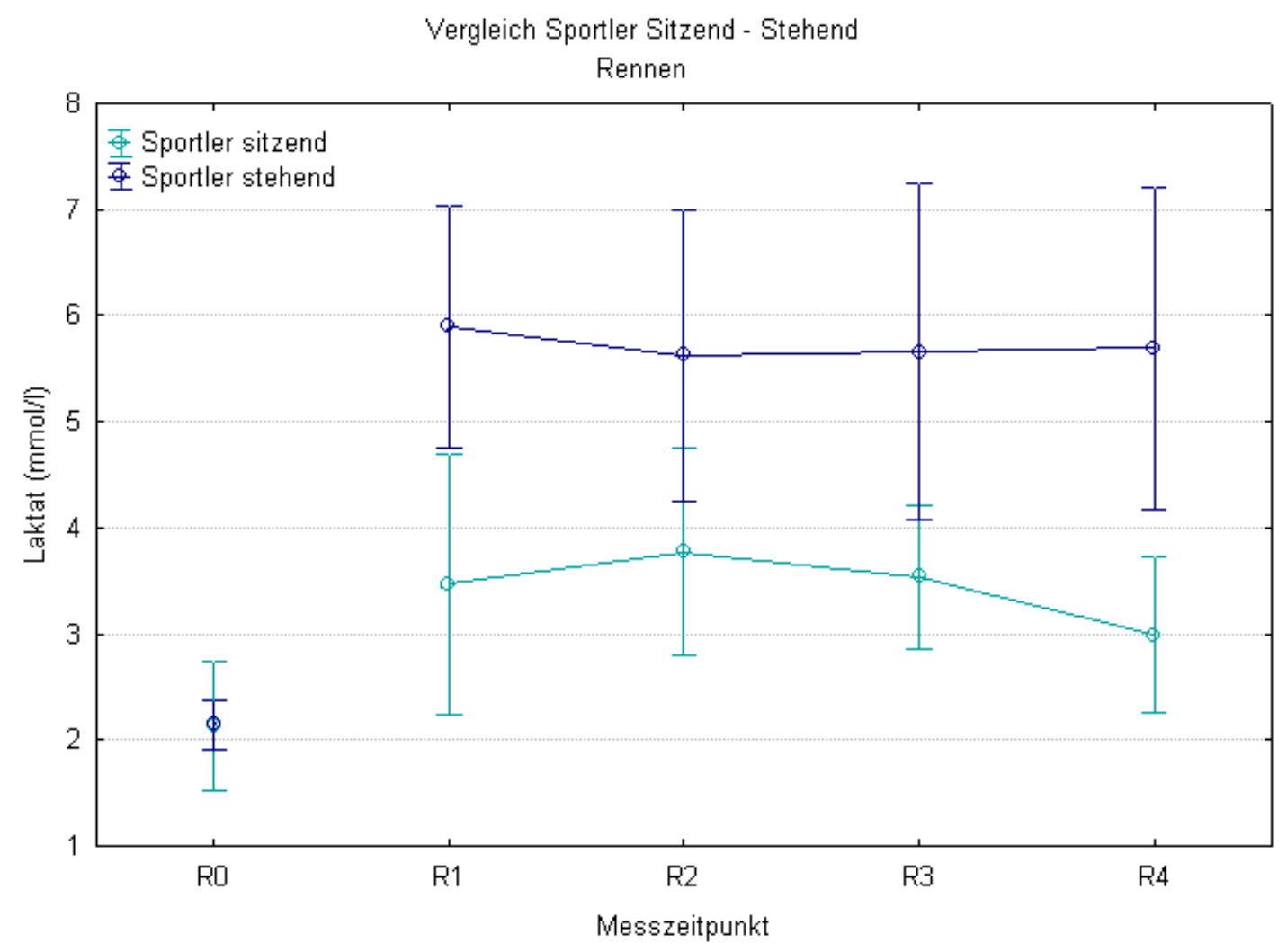

Abbildung 20: Athleten Rennen gesamt - Sitzend und Stehend

Bei der Gesamtbetrachtung von Renn- und Trainings-Messergebnissen sind die Laktatwerte innerhalb der Gruppe Sitzend signifikant niedriger als jene der stehenden Athleten $(\mathrm{p}=0,0018)$. Die Einzelwerte der Athleten der beiden Gruppen überschneiden sich kaum. Hinsichtlich des Verlaufs der Laktatkonzentration über die Zeit nach Belastung zeigt sich keine Signifikanz $(\mathrm{p}=0,5858)$. 
Den Wettkampf separat betrachtet besteht ebenfalls ein signifikanter Unterschied zwischen den Probandengruppen $(\mathrm{p}=0,019)$, ein Zeiteffekt ist jedoch auch im Rennen nicht nachzuweisen $(\mathrm{p}=0,38)$.

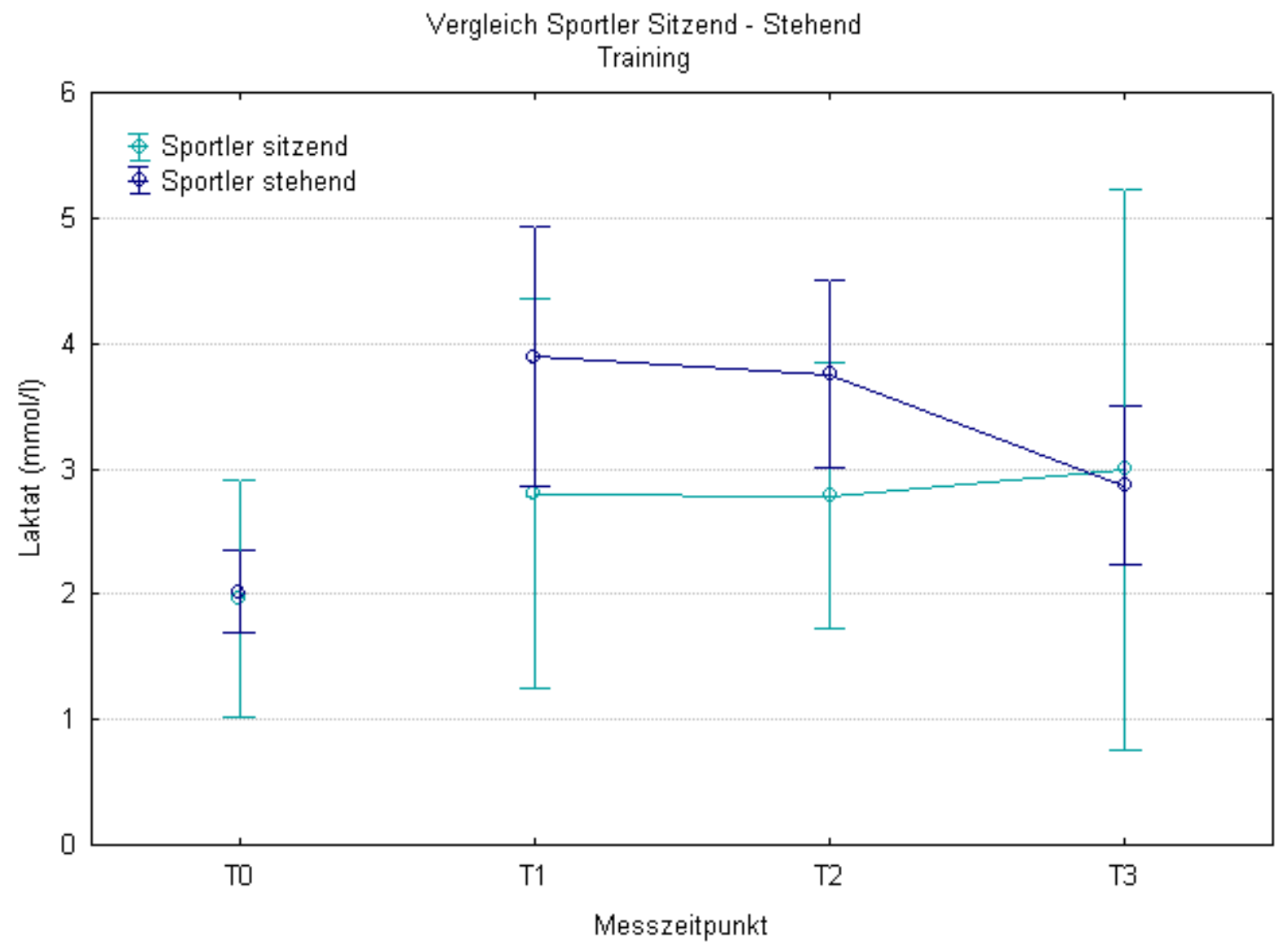

Abbildung 21: Athleten Training gesamt - Sitzend und Stehend

Diese Ergebnisse bestätigen sich in den Trainingsmessungen nicht. Insgesamt sind die im Training gemessenen Werte niedriger angesiedelt als jene nach dem Rennen.

Zwischen den bei den sitzenden und stehenden Athleten gemessenen Laktatkonzentrationen nach Trainingsende besteht kein signifikanter Unterschied $(p=0,599)$, jedoch ein signifikanter Zusammenhang des Verlaufs über die Zeit $(p=0,044)$. Damit unterscheiden sich die Ergebnisse sowohl von der Wettkampf- als auch von der Gesamtmessung.

Auffällig sind weiterhin die deutlich größeren Konfidenzintervalle verglichen mit denen in der Abbildung der Wettkampfergebnisse; der Vertrauensbereich ist also größer. Dies könnte auf stark um den Mittelwert streuende Einzelwerte oder ein zu kleines Probandenkollektiv zurückzuführen sein. 


\subsection{Erfolgreiche und weniger erfolgreiche Sportler}

Die einzelnen Laktatkonzentrationen streuen innerhalb einer Gruppe deutlich um den mittleren Laktatverlauf. In Anbetracht dessen wird im Anschluss ein möglicher Zusammenhang mit der Leistungsstärke eines Athleten untersucht.

Zunächst erfolgt eine Gegenüberstellung der Ergebnisse zweier Sportler einer Gruppe aus der Nachbelastungsphase der Wettkämpfe. In den Abbildungen sind die Mittelwertkurven (Mittelwert aller Einzelergebnisse) der Athleten dargestellt.

\subsubsection{Sitzende Sportler}

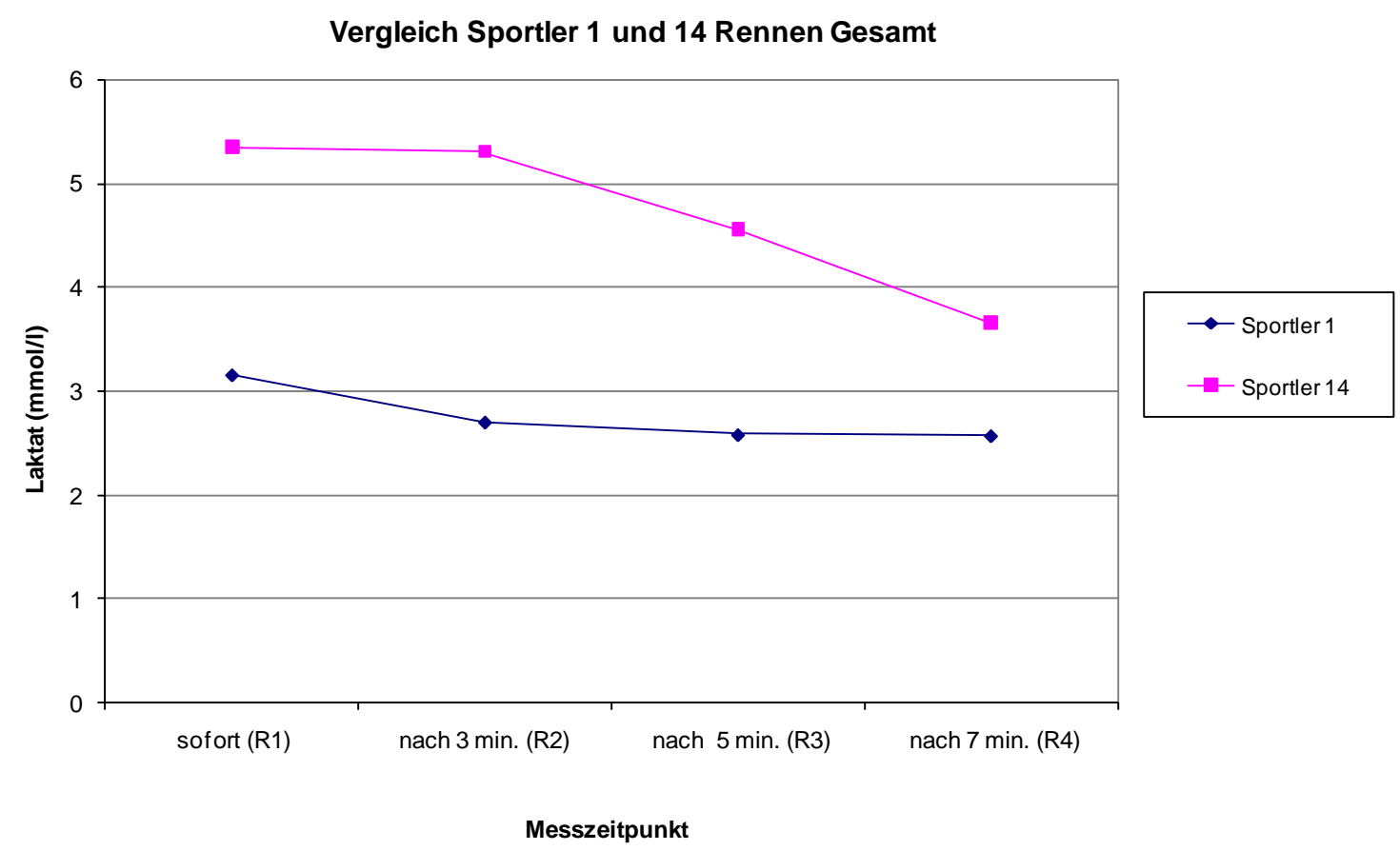

Abbildung 22: Vgl. Rennen Sportler 1 und 14

Exemplarisch werden Sportler 1 und 14 verglichen. Die Athleten starten in verschiedenen Wettkampfklassen (LW10/2 und LW11); auf Grund ähnlicher Schädigungsmuster ist ein Vergleich jedoch möglich. Zur Interpretation der Laktatkonzentration und ihrer Kinetik ist es sinnvoll, Sportler mit einer vergleichbaren Masse und Verteilung der Muskulatur auszuwählen. 
Sportler 1 erreicht in allen Messreihen zum Zeitpunkt R1 unmittelbar nach dem Rennen die maximale Laktatkonzentration im Blut; sie überschreitet nicht $4 \mathrm{mmol} / \mathrm{l}$ Blut und sinkt bereits während des Messzeitraums in der Nachbelastungsphase auf Niveau des Ruhewerts.

Sportler 14 weist insgesamt höhere Laktatkonzentrationen im Blut auf; sie sind nach Belastungsende rückläufig, die erhobenen Werte liegen jedoch zum Zeitpunkt R4 (nach 7 Minuten) noch oberhalb des Ruhewerts.

\subsubsection{Stehende Sportler}

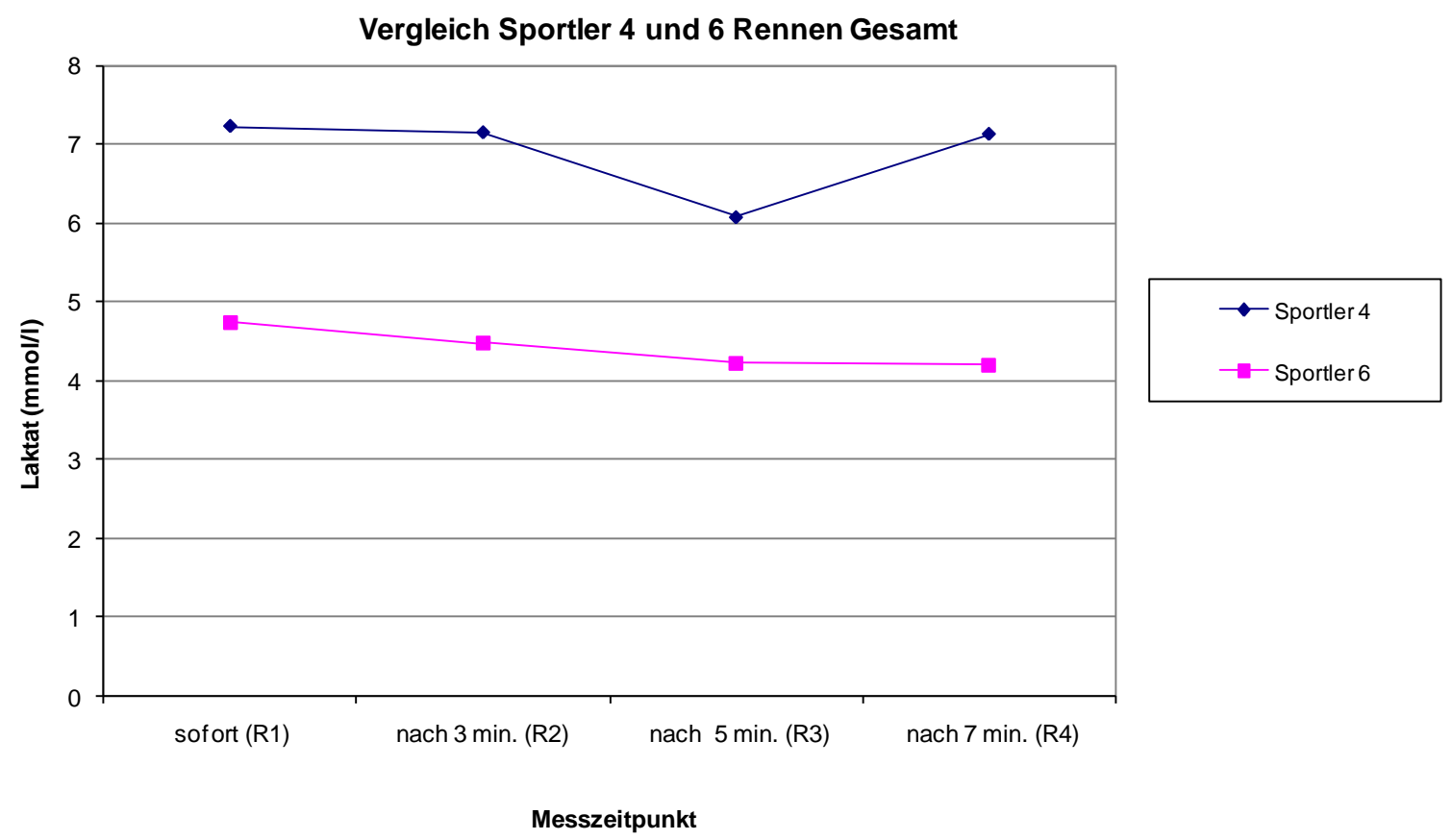

Abbildung 23: Vgl. Rennen Sportler 4 und 5

Sportler 4 weist an beiden Armen Amputationen auf; Sportler 5 ist einseitig beinamputiert. Ein Vergleich der Athleten ist schwierig, da sich die zur Bewegungsausführung geeignete Muskulatur unterscheidet. Allerdings liegen von den genannten Athleten viele Daten vor, so dass durch Kontinuität der Daten ihre Leistung besser zu beurteilen ist.

Sportler 4 erreicht zu unterschiedlichen Zeitpunkten nach Belastung die maximale Laktatkonzentration im Blut. Die Werte liegen mit bis zu 10,2 mmol/l deutlich höher als die der Probanden des sitzenden Kollektivs.

In drei von vier Messreihen im Rennen und in allen Trainingsmessungen im Winter fällt die Laktatkonzentrationskurve nach Erreichen des Maximums ab; innerhalb des Messzeitraums wird das Ruhewertniveau nicht erreicht. In Jahr 2 liegt zum Zeitpunkt R3 ein deutlich 
niedrigerer Wert als in den anderen Messreihen vor, ansonsten sind die Verläufe des Laktats nach Belastung relativ stabil. Man könnte einen falsch niedrigen Wert vermuten.

Die Mittelwertkurve wäre dann zum Zeitpunkt R3 verzerrt; zwischen R2 und R4 ist ein Abfall der Laktatkonzentration zu beobachten.

In Abb. 23 sind die mittleren Verläufe der Laktatkonzentration dargestellt.

Sportler 5 zeigt hohe Laktatkonzentrationen im Blut in Ruhe, die Werte steigen nach Belastungsende bis maximal 10,7 mmol/l an (Jahr $1 \mathrm{R} 3$ ); nach kurzem Abfall der Kurve nach Erreichen des Maximums steigt sie zum Teil erneut an.

Insgesamt sind die Laktatkonzentrationen im Blut bei Sportler $5 \mathrm{zu}$ allen Zeitpunkten höher als jene bei Sportler 4 gemessenen. Beide Athleten erreichen während des Messzeitraums nicht Ruhewertniveau.

In Ergänzung wird ein weiterer Sportler, der ein vergleichbares Schädigungsmuster wie Sportler 4 aufweist, aufgeführt.

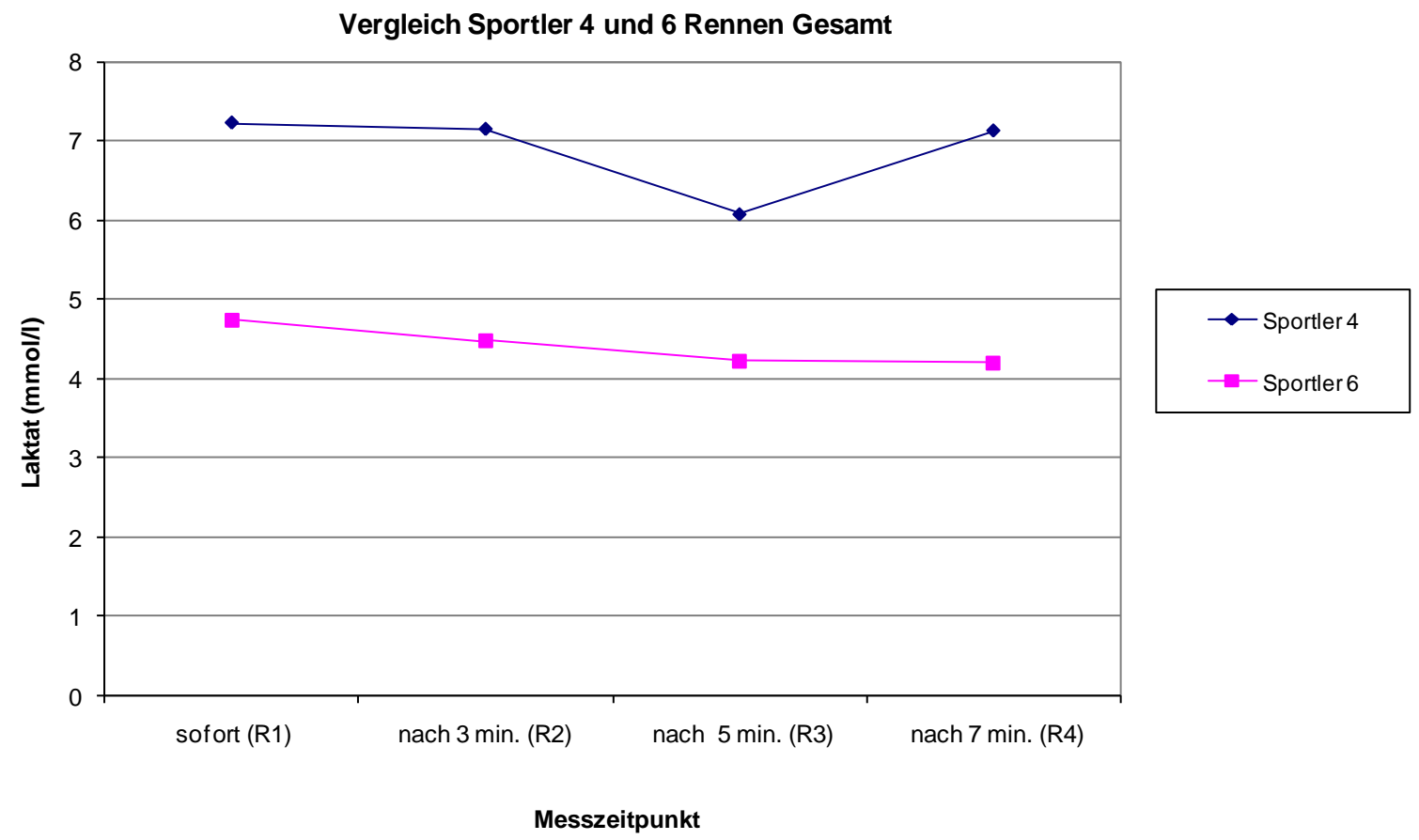

Abbildung 24: Vgl. Rennen Sportler 4 und 6

Die maximal gemessenen Laktatkonzentrationswerte im Blut von Sportler 6 erreichen nicht das Niveau von Sportler 4; während der Nachbelastungsphase ist ein Abfall der Laktatkonzentration zu beobachten. Auch er erreicht innerhalb des Messzeitraums nicht Ruhewertniveau. 


\subsubsection{Sitzende vs. Stehende Sportler}

Zunächst erfolgt die Gegenüberstellung zweier erfolgreicher Athleten: Sportler 1 sitzend und Sportler 4 stehend. Anschließend werden die Gruppen Sitzend und Stehend insgesamt verglichen.

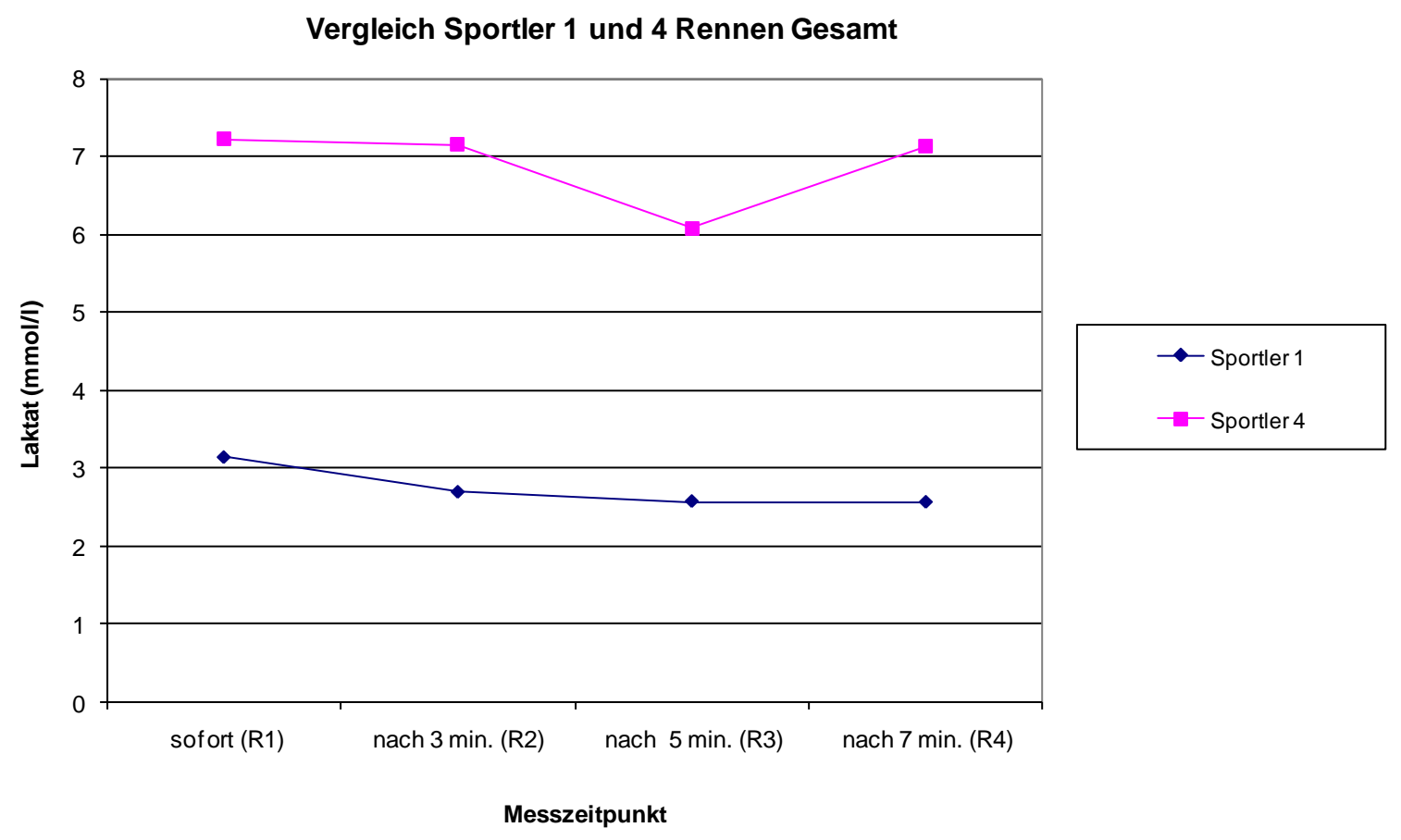

Abbildung 25: Vgl. Rennen Sportler 1 und 4

Vergleicht man zwei ausgewählte Sportler (Sportler 1 und 4) aus den Gruppen Sitzend und Stehend, zeigt sich ein deutlicher Unterschied hinsichtlich der Laktatkonzentration im Blut nach Belastung.

Bei beiden Athleten ist ein deutlicher Anstieg der Laktatkonzentration im Blut während der Belastung im Rennen zu erkennen, anschließend ist sie in engem zeitlichen Zusammenhang zum Beenden der Belastung rückläufig.

Sportler 4 als stehender Athlet erreicht deutlich höhere Laktatkonzentrationen, die Kurven in drei von vier Messreihen fallen nach Belastungsende ab, er erreicht jedoch während des Messzeitraums nicht Ruhewertniveau.

Sportler 1 zeigt niedrigere Maximalwerte als Sportler 4, die vor Belastungsbeginn bestimmten Ruhewerte unterscheiden sich kaum; weiterhin sind die Werte rückläufig bis auf Niveau der Ruhewerte. 
Beim Vergleich innerhalb der Gruppen wurden Sportler ausgewählt, die unterschiedlich erfolgreich im Wettkampf waren: Sportler 1,3,4,6,7 und 8 erfolgreich (= Platzierung innerhalb der ersten zehn Plätzen); Sportler 2,5,13 und 14 weniger erfolgreich (= Platzierung ab dem elften Rang).

Es zeigen sich Unterschiede in der Laktatkonzentration und ihrem Verlauf über die Zeit.

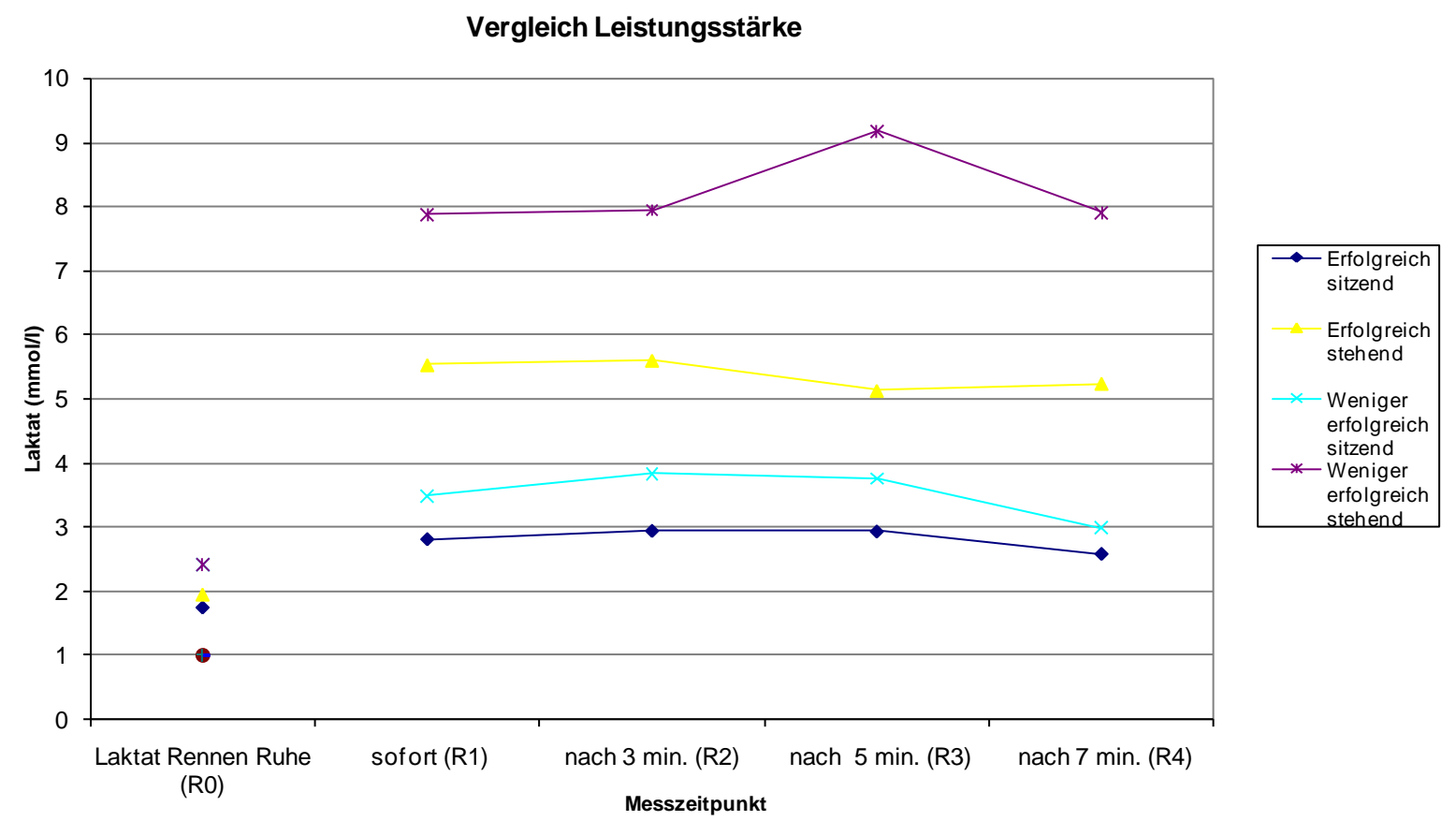

Abbildung 26: Vgl. erfolgreiche/ weniger erfolgreiche Sportler - Sitzend und Stehend

In Abbildung 26 zeigen sich verschiedene Verläufe der Laktatkonzentration:

Erfolgreich sitzend: Niedrigere Laktatkonzentrationen, nach 7 Minuten fast Ruhewertniveau erreicht.

Weniger erfolgreich sitzend: Niedrigere Laktatkonzentrationen als stehende Gruppe, höhere Konzentrationen als erfolgreiche sitzende Sportler, nach 7 Minuten fast Ruhwertniveau erreicht.

Erfolgreich stehend: Höhere Laktatkonzentrationen als sitzende Gruppe, nach 7 Minuten nicht Ruhewertniveau erreicht.

Weniger erfolgreich stehend: Höchste Laktatkonzentration, nach 7 Minuten nicht Ruhewertniveau erreicht.

Insgesamt zeigt sich eine große Streuung der Werte zu den jeweiligen Messzeitpunkten. 
Vergleich Erfolgreich - Weniger erfolgreich Sitzend

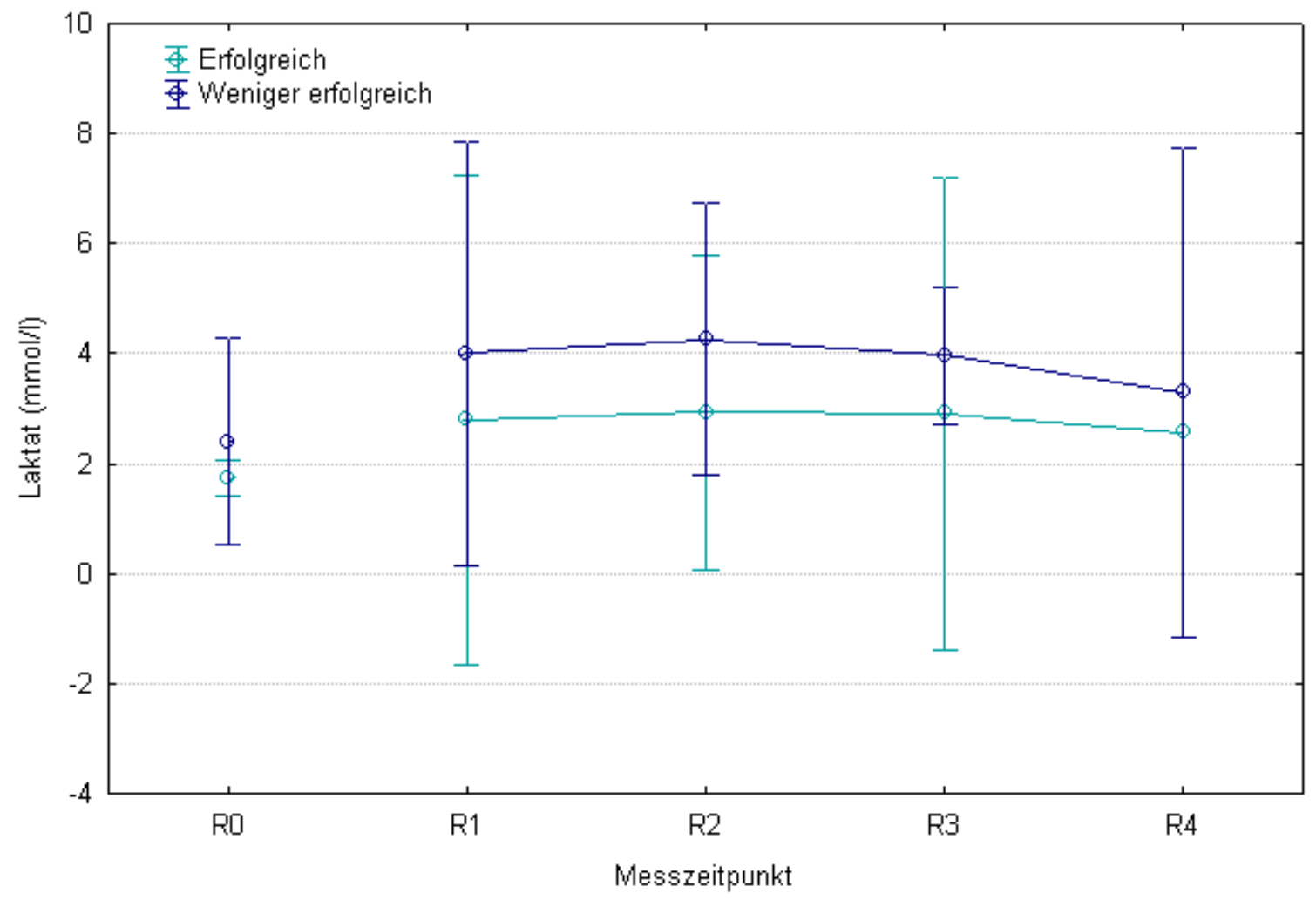

Abbildung 27: Sitzende Sportler: Erfolgreich - weniger erfolgreich

Vergleich Erfolgreich - Weniger erfolgreich

Stehend

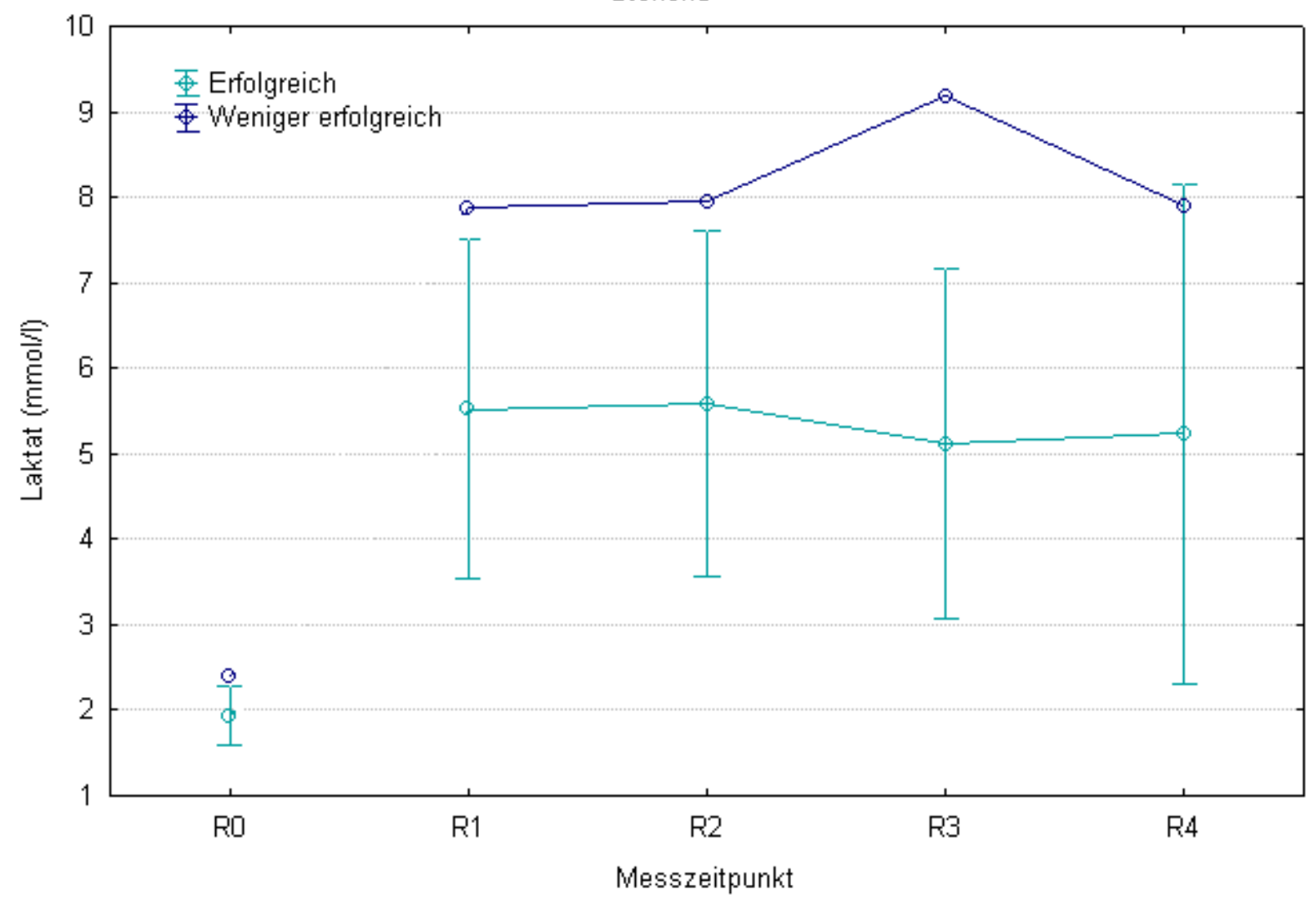

Abbildung 28: Stehende Sportler: Erfolgreich - weniger erfolgreich 
In den einzelnen Gruppen zeigt sich ein Abfall der Laktatkonzentration im Blut über die Zeit (signifikanter Zeiteffekt (Sitzend $\mathrm{p}=0,02 /$ Stehend $\mathrm{p}<0,001)$ ). Unterschiede der Höhe der Laktatkonzentration nach Belastung zwischen erfolgreichen und nicht erfolgreichen Sportlern waren in beiden Gruppen nicht signifikant nachzuweisen (Sitzend $\mathrm{p}=0,082 /$ Stehend $\mathrm{p}=$ 0,158); es ist jedoch der Trend festzustellen, dass weniger erfolgreiche Sportler eher höhere Laktatkonzentrationen aufweisen.

Beim Vergleich der Gruppen sitzend und stehend besteht ein signifikanter Unterschied hinsichtlich der Höhe der Laktatkonzentration $(\mathrm{p}=0,035)$, ein Zusammenhang mit der Leistungsstärke ist jedoch nicht eindeutig nachweisbar (Zeiteffekt $p=0,173$ / Status $p=0,108$ ).

\subsection{Kurvenanpassungsverfahren}

Als Auswertungsmodell für die Laktatkonzentration über die Zeit nach Belastung stehen verschiedene Modelle zur Verfügung.

Ziel ist es, eine Funktion zu konstruieren, über die das Ausschwingverhalten des Laktats nach Belastung beschreiben wird. Auf Grund der physiologischen Gegebenheiten (Produktion, Verteilung und Elimination des Laktats) wäre eine Modellierung der Laktatkonzentrationskurve über eine Polynom- oder Exponentialfunktion über die Zeit denkbar. Des Weiteren soll der Versuch unternommen werden, einen Wert y zu einem bestimmten Zeitpunkt $\mathrm{x}$ zu berechnen (Zeitpunkt $\mathrm{x}=$ nach 4 Minuten), zu dem keine Messergebnisse vorliegen; im Folgenden benannt als y-Polynom und y-Exponential.

Anhand einiger Beispiele an ausgewählten Probanden erfolgt ein Vergleich der zwei genannten Darstellungsformen Polynom (3. Ordnung) und Exponentialfunktion.

In der Annahme, die Laktatkonzentration im Blut erreiche innerhalb eines Zeitraums von maximal einer Stunde wieder Ruhewertniveau, wird der Ruhewert R0 als Zeitpunkt R5 in der Grafik dargestellt. 


\subsubsection{Sportler 1}

a) Polynom

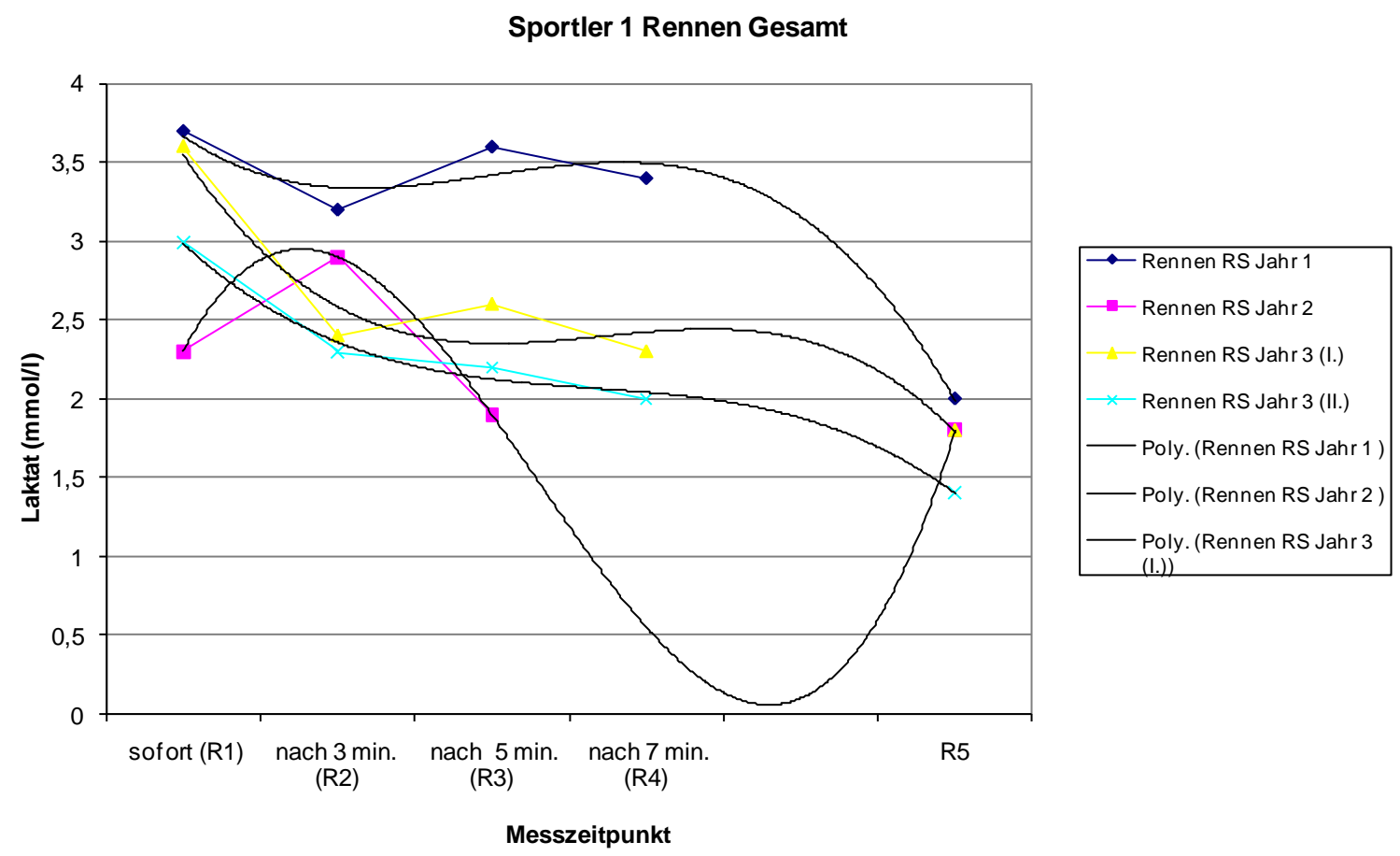

Abbildung 29: Sportler 1 Rennen gesamt - Polynome

\section{Analytischer Ausdruck}

RS $1 \quad y=-0,068 x^{3}+0,612 x^{2}-1,684 x+4,803$

RS $2 \quad y=0,208 x^{3}-2,05 x^{2}+5,291 x-1,15$

RS 3 I $\quad y=-0,070 x^{3}+0,789 x^{2}-2,838 x+5,669$

RS 3 II $\quad y=-0,039 x^{3}+0,429 x^{2}-1,639 x+4,234$

Mittelwert $y=-0,043 x^{3}+0,433 x^{2}-1,455 x+4,217$

\section{Beispielrechnung $(x=4)$}

RS 1

RS 2

$y=-0,068\left(4^{3}\right)+0,612\left(4^{2}\right)-1,684(4)+4,803=3,5$

RS 3 I

$\mathrm{y}=0,208\left(4^{3}\right)-2,05\left(4^{2}\right)+5,291(4)-1,15$

$=0,524$

RS 3 II

$\mathrm{y}=-0,070\left(4^{3}\right)+0,789\left(4^{2}\right)-2,838(4)+5,669=2,46$

Mittelwert

$$
\mathrm{y}=-0,039\left(4^{3}\right)+0,429\left(4^{2}\right)-1,639(4)+4,234=2,05
$$

$\mathrm{y}=-0,043\left(4^{3}\right)+0,433\left(4^{2}\right)-1,455(4)+4,217=2,58$ 
b) Exponentialfunktion

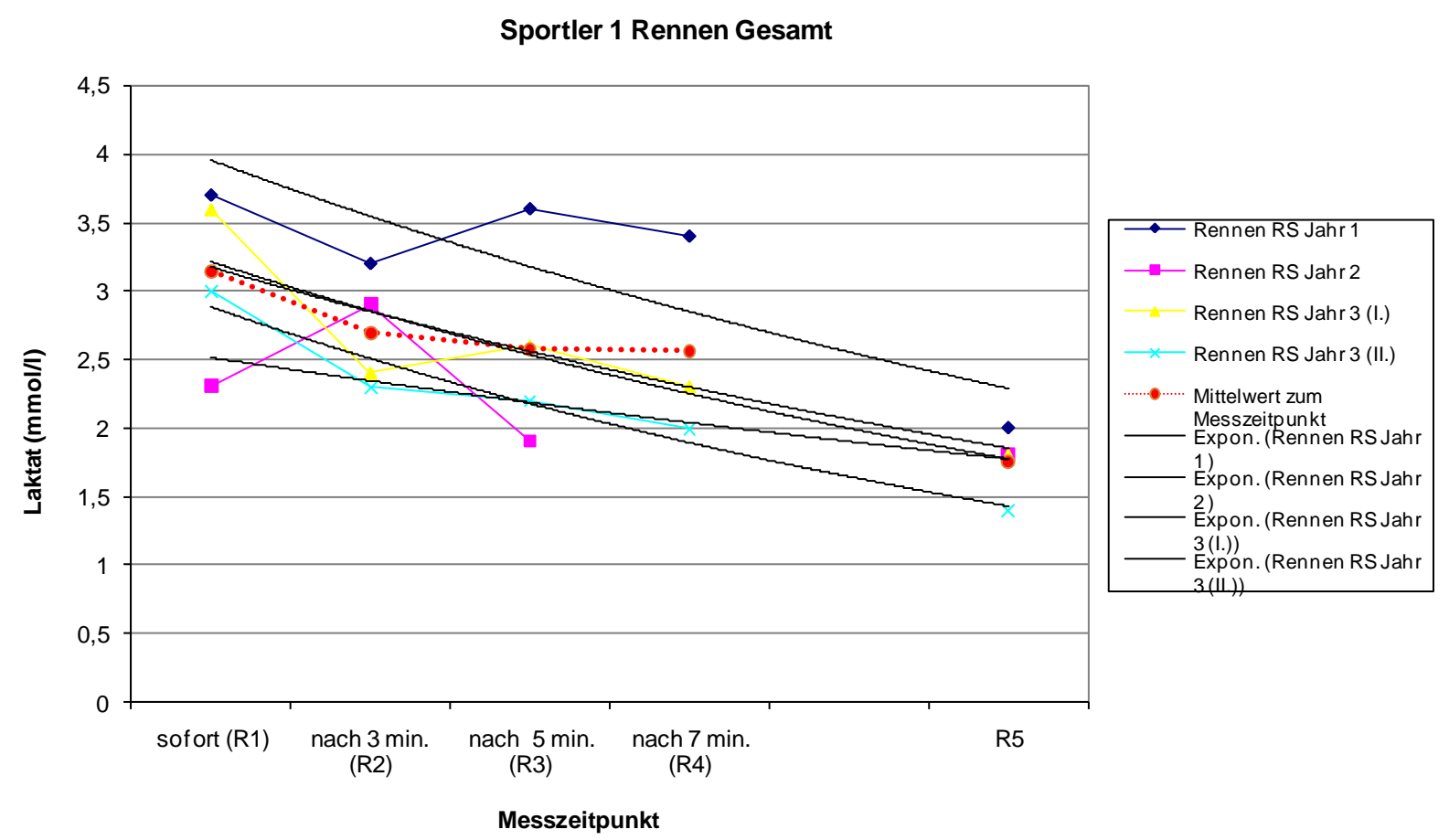

Abbildung 30: Sportler 1 Rennen gesamt - Exponentialfunktionen

\section{Analytischer Ausdruck}

RS $1 \quad y=4,402 e^{-0,10 x}$

RS $2 y=2,688 e^{-0,06 x}$

RS3 I $y=3,612 e^{-0,11 x}$

RS3 II $\quad y=3,317 e^{-0,14 x}$

Mittelwert $\mathrm{y}=3,526 \mathrm{e}^{-0,10 \mathrm{x}}$

\section{Beispielrechnungen $(x=4)$}

RS1

$$
\mathrm{y}=2,951
$$

RS2

$\mathrm{y}=2,114$

RS3 I

$\mathrm{y}=2,326$

RS3 II

$\mathrm{y}=1,895$

Mittelwert $\quad \mathrm{y}=2,364$

Die berechneten Werte zum Zeitpunkt $\mathrm{x}=4$ Minuten variieren in Abhängigkeit von der angewandten Funktion. Insgesamt sind die über das Polynom bestimmten Laktatkonzentrationen höher als jene über die Exponentialfunktion berechneten. Bei Betrachtung der Relation zu den gemessenen Werten zu den Zeitpunkten R2 (3 Minuten) und R3 (5 Minuten) entspricht y-Polynom eher dem Verlauf, y-Exponential ist niedriger angesiedelt. 


\subsubsection{Sportler 4}

a) Polynom

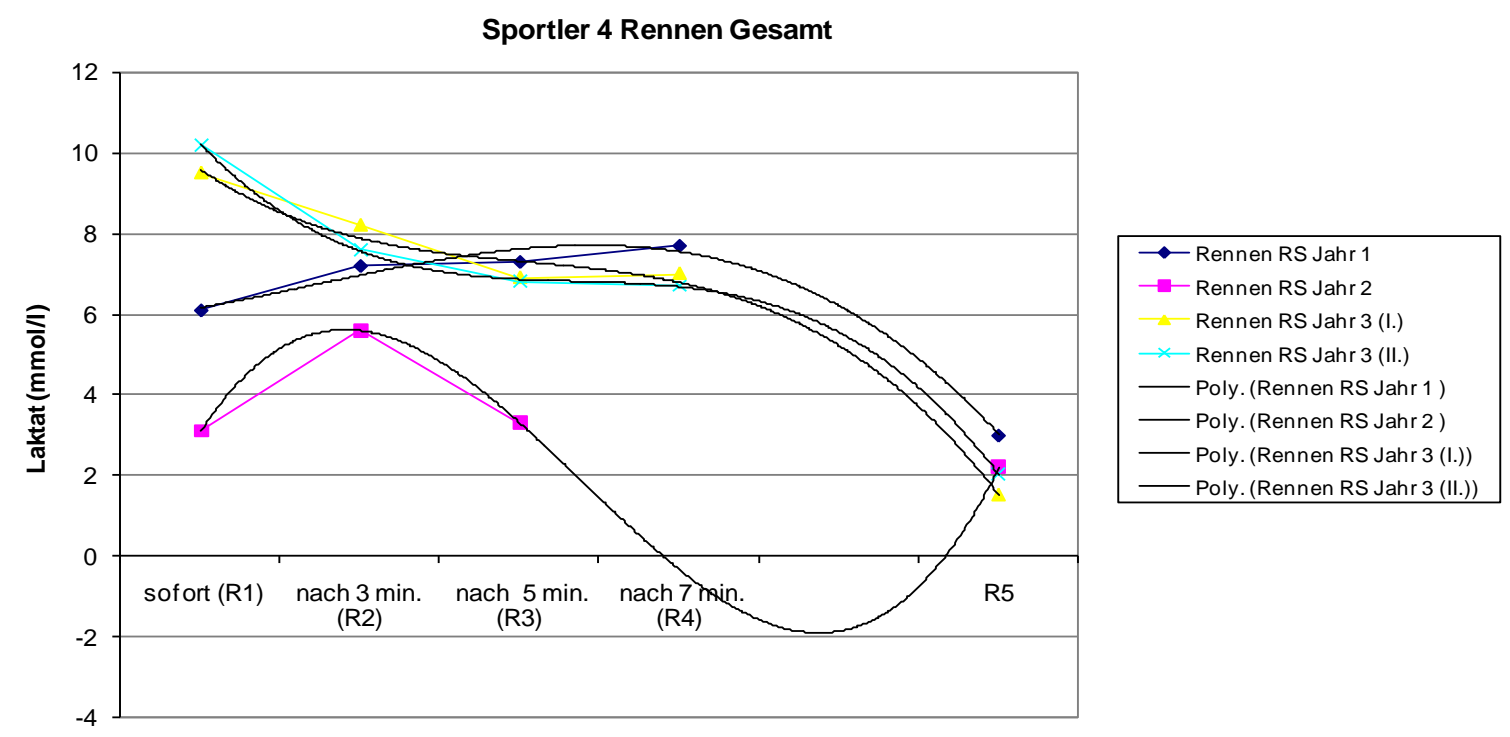

Messzeitpunkt

Abbildung 31: Sportler 4 Rennen gesamt - Polynome

\section{Analytischer Ausdruck und Beispielrechnung ( $\mathrm{x}=4)$}

RS1

RS2

RS3 I

RS3 II

$$
\mathrm{y}=-0,093 \mathrm{x}^{3}+0,482 \mathrm{x}^{2}+0,009 \mathrm{x}+5,764
$$$$
y=0,576 x^{3}-5,86 x^{2}+16,04 x-7,66
$$$$
y=-0,181 x^{3}+1,649 x^{2}-5,379 x+13,49
$$$$
y=-0,242 x^{3}+2,432 x^{2}-8,26 x+16,28
$$

$$
=7,56
$$

$=1,604$

$=6,77$

$=6,66$

Mittelwert $\quad \mathrm{y}=-0,173 \mathrm{x}^{3}+1,484 \mathrm{x}^{2}-3,982 \mathrm{x}+10,03=6,77$ 
b) Exponential

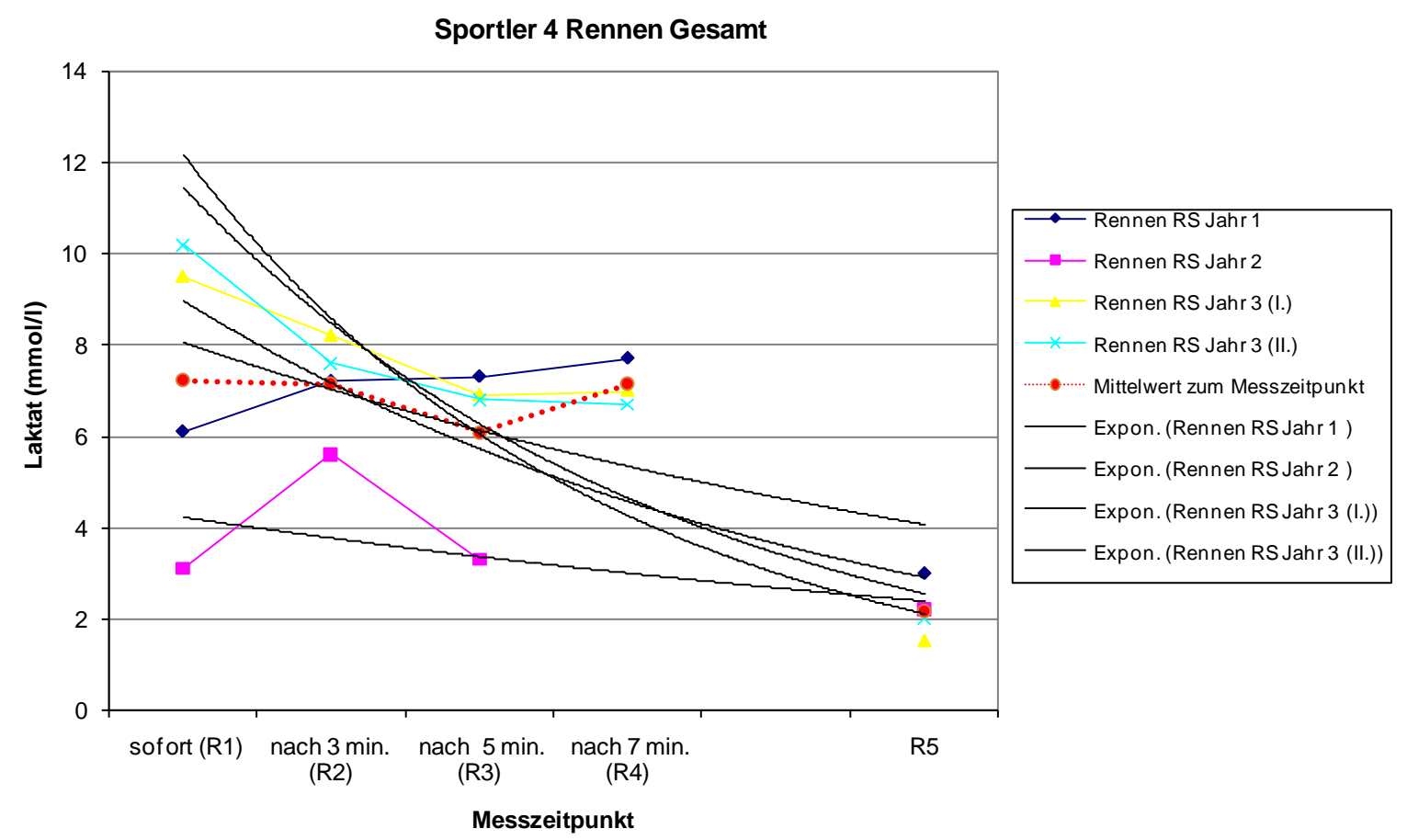

Abbildung 32: Sportler 4 Rennen gesamt - Exponentialfunktionen

\section{Analytischer Ausdruck und Beispielrechnung ( $x=4)$}

RS1 $y=9,227 \mathrm{e}^{-0,13 x}$

$\mathrm{y}=5,49$

RS2 $y=4,741 e^{-0,11 x}$

$\mathrm{y}=3,05$

RS3 I $y=17,21 e^{-0,34 x}$

$\mathrm{y}=4,42$

RS3 II $y=15,45 e^{-0,30 x}$

$\mathrm{y}=4,65$

Mittelwert $y=11,21 e^{-0,22 x}$

$\mathrm{y}=4,65$

Auch bei Sportler 4 zeigt sich, dass der über das Polynom berechnete Wert y höher ist als der über die Exponentialfunktion bestimmte.

Ähnlich wie bei Sportler 1 gilt, dass das y-Polynom dem Verlauf der gemessenen Laktatkonzentration in der Nachbelastungsphase näher kommt.

Bemerkenswert ist, dass bei beiden Athleten - 1 und 4 - im Riesenslalomrennen im zweiten Jahr über die Polynomfunktion sehr niedrige y-Werte ermittelt wurden, die deutlich vom sonstigen Verlauf der Laktatwerte abweichen. Auch die Graphen in Jahr 2 (drei Nachbelastungswerte) scheinen fehlerhaft. Bei Sportler 1 fällt das Polynom deutlich ab, bei Sportler 4 werden sogar negative Werte erreicht. 


\subsubsection{Sportler 5}

a) Polynom

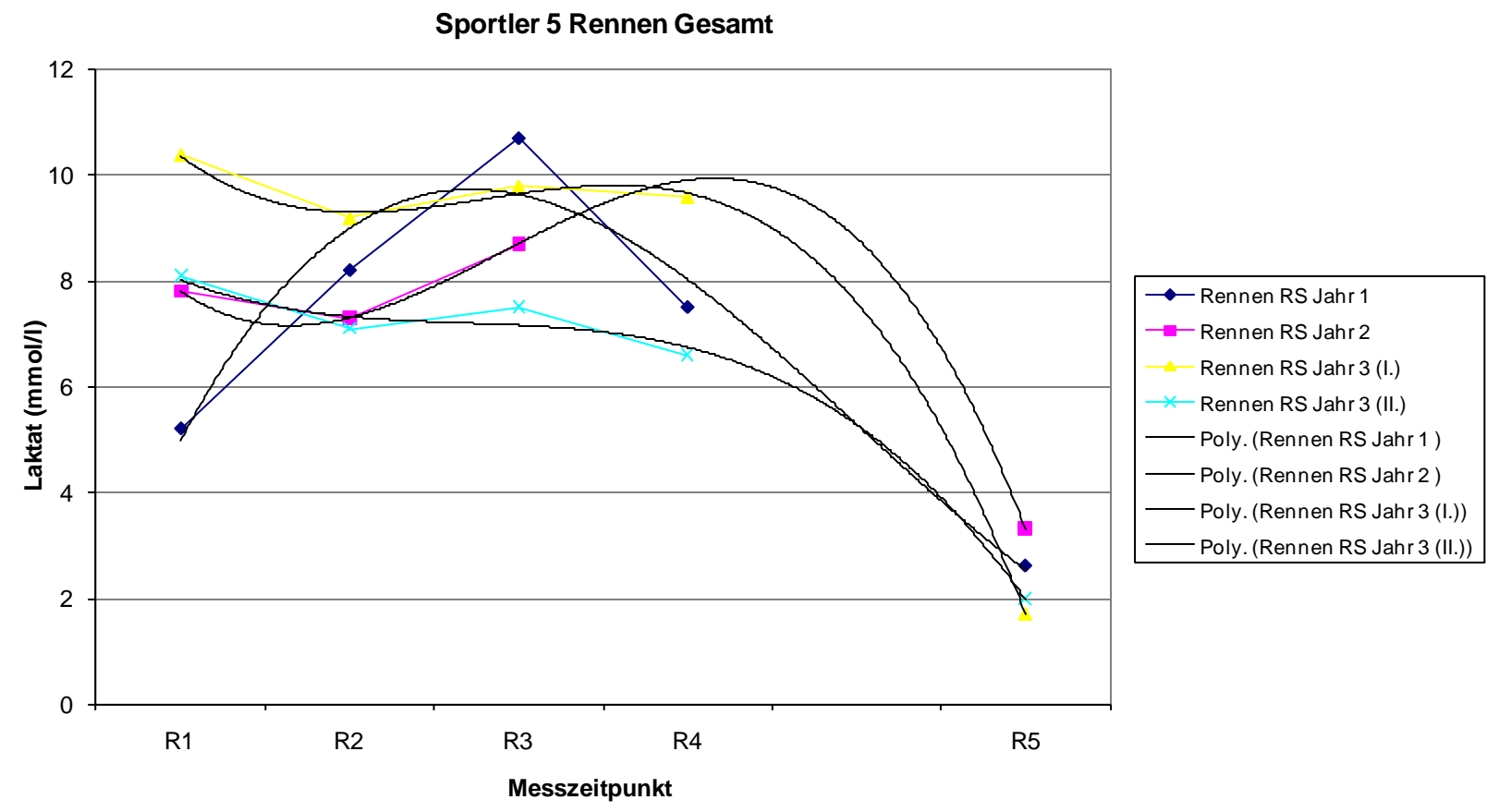

Abbildung 33: Sportler 5 Rennen gesamt - Polynome

\begin{tabular}{|c|c|c|}
\hline \multicolumn{3}{|c|}{ Analytischer Ausdruck und Beispielrechnung $(x=4)$} \\
\hline RS1 & $y=0,186 x^{3}-2,802 x^{2}+11,11 x-3,507$ & $y=8,01$ \\
\hline $\mathrm{RS} 2$ & $y=-0,35 x^{3}+3,05 x^{2}-7,2 x+12,3$ & $y=9,9$ \\
\hline RS3 I & $y=-0,291 x^{3}+2,457 x^{2}-6,394 x+14,6$ & $y=9,71$ \\
\hline RS3 II & $y=-0,131 x^{3}+1,058 x^{2}-2,947 x+10,05$ & $y=6,81$ \\
\hline Mittelwert & $y=-0,096 x^{3}+0,456 x^{2}-0,130 x+7,541$ & \\
\hline
\end{tabular}




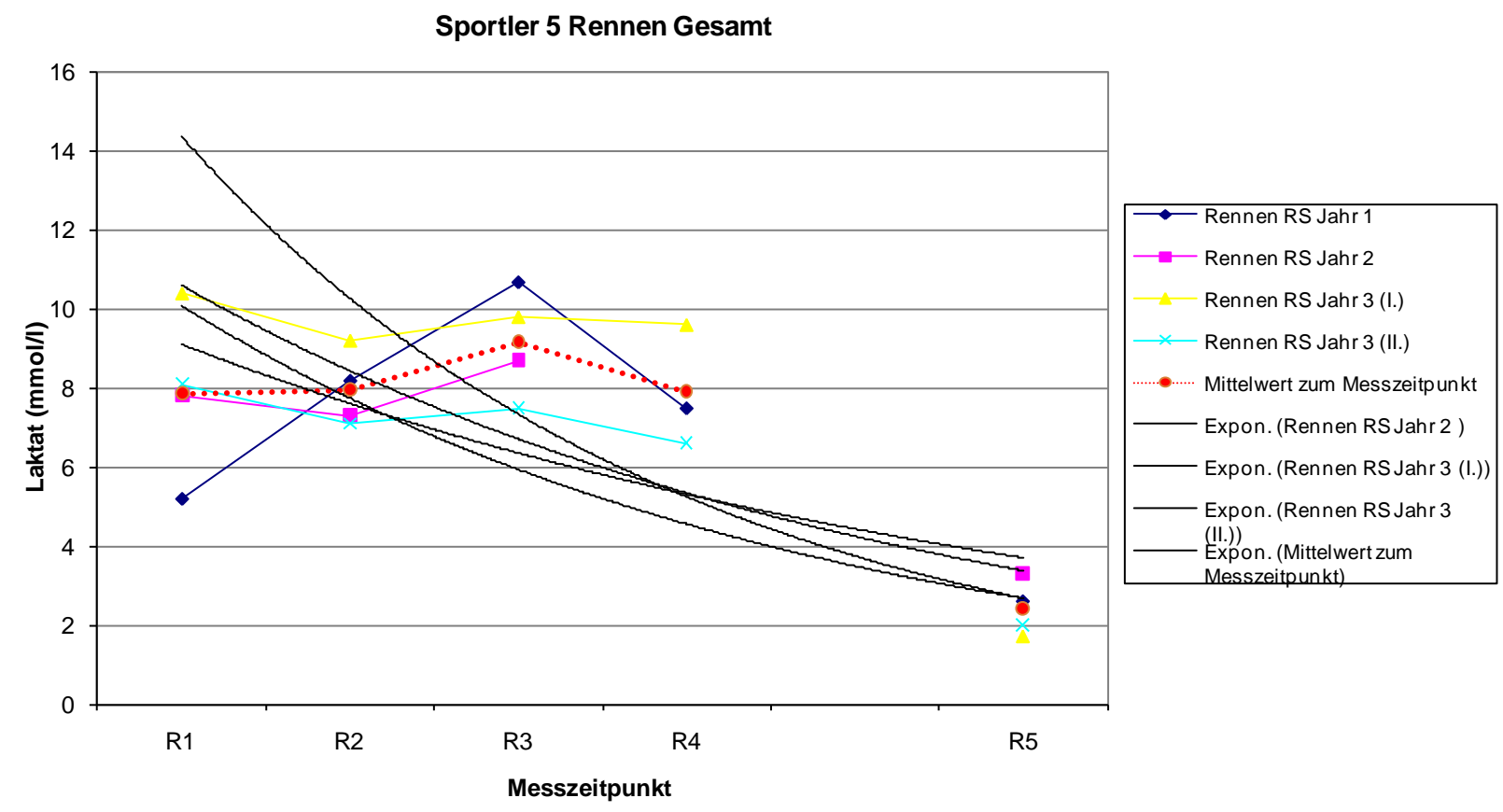

Abbildung 34: Sportler 5 Rennen gesamt - Exponentialfunktionen

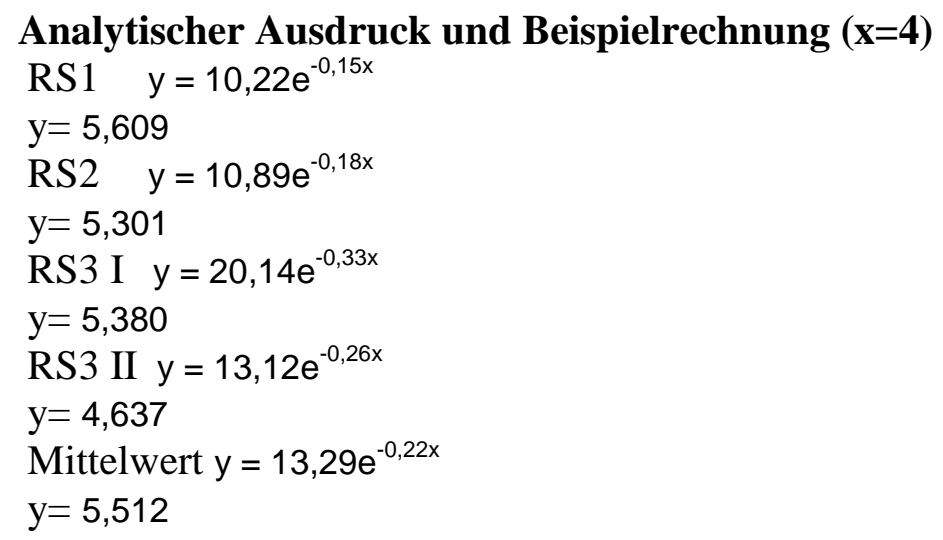

Insgesamt zeigen sich große Schwankungen der Werte um den Mittelwert. Auch bei Sportler 5 fallen die über die Exponentialfunktion berechneten Laktatkonzentrationen zum Zeitpunkt $\mathrm{x}=4$ niedriger aus. $\mathrm{y}$-Polynom ist dem Verlauf in den jeweiligen Jahren näher.

Beim Vergleich der beiden Kurvenanpassungsverfahren liegen bei allen Athleten die über das Polynom der 3. Ordnung berechneten Laktatkonzentrationen höher als jene über die Exponentialfunktion ermittelten; weiterhin nähern sie sich der Laktatkurve stärker an.

Bei allen Beispielen zeigen sich Abweichungen in RS II, v.a. bei den Athleten 1 und 4. Bei Sportler 1 differieren y-Polynom und y-Exponential weniger; die Unterschiede sind bei 
Sportler 4 und 5, die insgesamt höhere Laktatkonzentrationen nach Belastung entwickeln, ausgeprägter.

Im Fall der Athleten 1 und 4 sind weniger starke Abweichungen der berechneten yEinzelwerte vom berechneten y-Mittelwert zu beobachten als bei Sportler 5; diese Tendenz spiegelt sich bereits in der Einzelfallbetrachtung wider.

Abschließend wird ein Vergleich der erhobenen Daten der Behindertensportler mit einer gesunden Probandengruppe vorgenommen.

\subsection{Vergleich Athleten Behindertensport und gesunde Vergleichsgruppe}

Es wird die Fragestellung behandelt, ob Unterschiede der Laktatkonzentration nach Belastung zwischen behinderten und nichtbehinderten Sportlern bestehen.

In der folgenden Abbildung sind die Gruppen Sitzend, Stehend, Gesunde Männer und Gesunde Frauen abgebildet.

Die Werte der gesunden Vergleichsgruppe sind der Arbeit von Temme (2001) (Riesenslalom) entnommen. Die Messzeitpunkte sind im Hinblick auf eine Vergleichbarkeit extrapoliert.

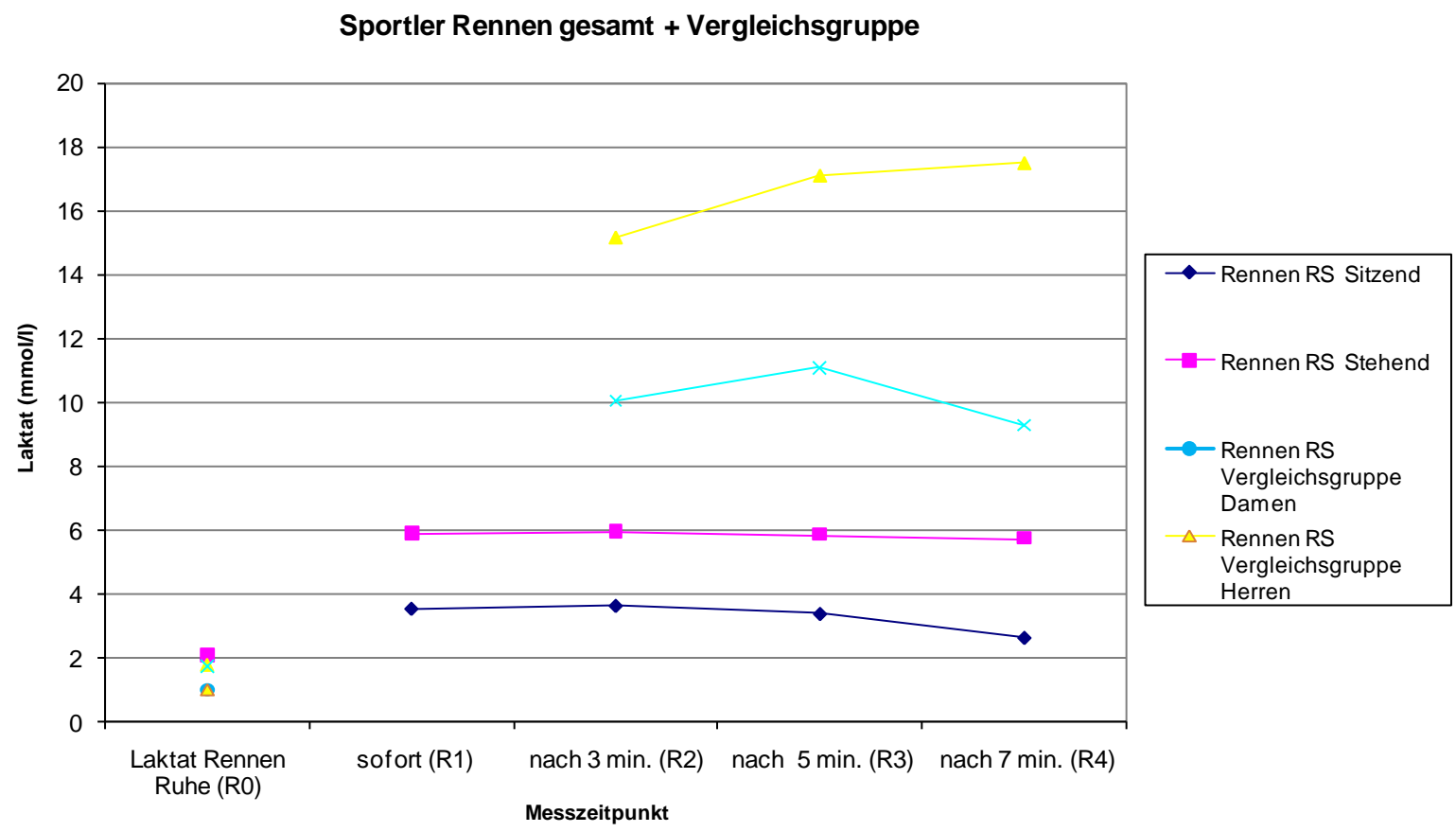

Abbildung 35: Vgl. behinderte Sportler und gesunde Vergleichsgruppe 
Die Blutlaktatkonzentrationen sind unterschiedlich hoch, vereinfacht dargestellt:

\section{Sitzend< Stehend< gesunde Frauen< gesunde Männer.}

Alle Gruppen zusammengenommen besteht ein signifikanter Unterschied in der Höhe der Laktatkonzentration $(\mathrm{p}<0,001)$, weiterhin besteht ein signifikanter Zusammenhang hinsichtlich des Verlaufs (Zeiteffekt $\mathrm{p}=0,03$ ).

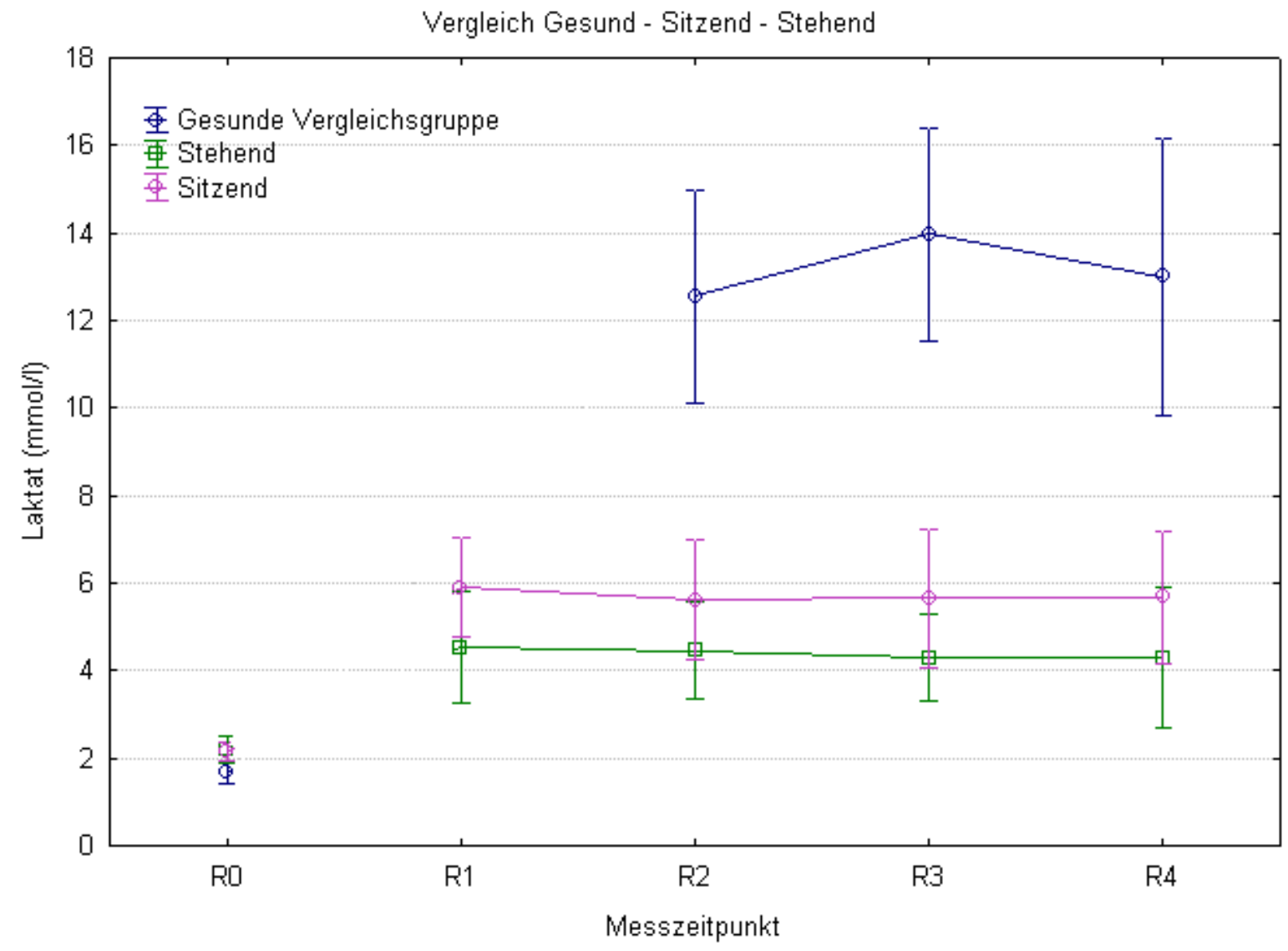

Abbildung 36: Vgl. Gesund - Sitzend - Stehend 
Die gesunde Vergleichsgruppe zusammengefasst (siehe Abb. 36), besteht im Vergleich zu sitzenden und stehenden Athleten ein signifikanter Unterschied in der Laktatkonzentration.

Bei einem Vergleich von stehenden und gesunden Probanden zeigen sich deutliche Unterschiede in der Laktatkonzentration; die gesunden Probanden erreichen signifikant höhere Werte als die stehenden Athleten $(\mathrm{p}<0,001)$. Der Verlauf der Laktatkonzentration nach Belastung ist vergleichbar $(\mathrm{p}<0,001)$.

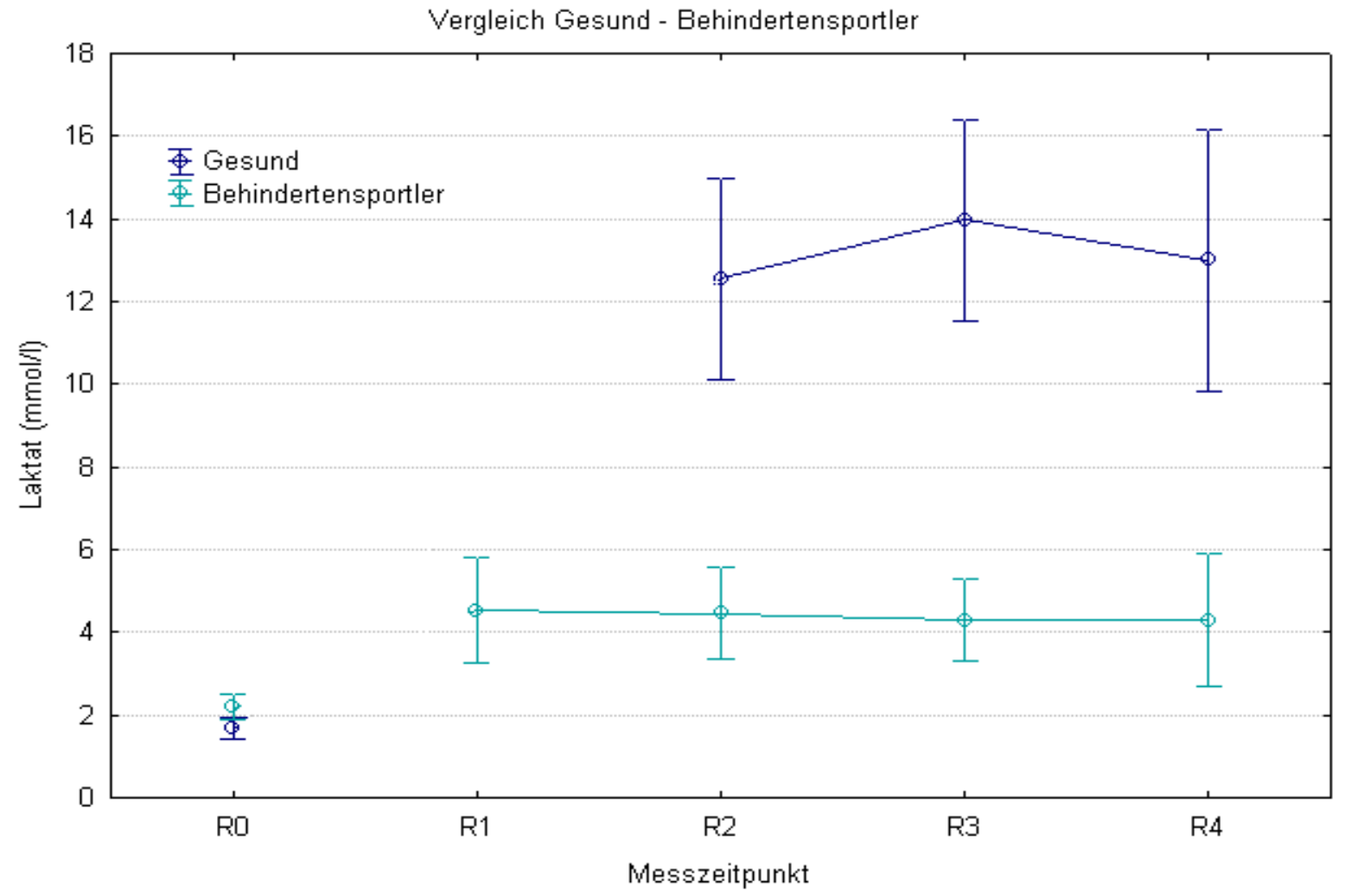

Abbildung 37: Vgl. Gesund - Behindertensportler

Ein abschließender Vergleich zwischen Behinderten- und Nichtbehindertensportlern zeigt, dass die gesunden Athleten deutlich höhere Laktatkonzentrationen erreichen; die Streuung der Einzelwerte um den Mittelwert ist in beiden Gruppen ausgeprägt. Die Konfidenzintervalle in der gesunden Vergleichsgruppe sind größer als jene der Behindertensportler; der Vertrauensbereich ist also größer. 


\section{Diskussion}

Inhalt der Arbeit ist eine trainingsbegleitende Leistungsdiagnostik im Behindertensport Ski alpin.

Folgende Fragen wurden berücksichtigt:

- Ist Laktat als leistungsdiagnostischer Parameter für sportartspezifische Untersuchungen im Riesenslalom geeignet?

- Zeigen sitzende und stehende Athleten Unterschiede in der Höhe der Laktatkonzentration oder eine andere Laktatkinetik nach Belastung?

- Zeigen erfolgreiche und weniger erfolgreiche Sportler Unterschiede in der Höhe der Laktatkonzentration oder eine andere Laktatkinetik nach Belastung?

- Lassen sich Parallelen oder Unterschiede hinsichtlich der Laktatkonzentration zu gesunden Athleten feststellen?

- Ist es möglich, trotz der Heterogenität der Probandengruppe aus den leistungsdiagnostischen Untersuchungen im Feldversuch Schlussfolgerungen für eine Trainingssteuerung bzw. -planung zu ziehen?

- Leistungsdiagnostische Untersuchungen im Behindertensport Ski alpin sind im Feldversuch praktikabel und aussagekräftig hinsichtlich ihrer Verwendung in der Trainingssteuerung.

\subsection{Diskussion der Methode}

Im Rahmen der vorliegenden Studie wurden sportartspezifische leistungsdiagnostische Untersuchungen in Form eines Feldversuchs am Skihang durchgeführt. Als Probanden fungierten vierzehn Athleten aus dem Behindertenleistungssportbereich mit verschiedenen physischen Beeinträchtigungen; sechs Sportler starteten in der Kategorie sitzend, acht Athleten in der Kategorie stehend. Diese Unterteilung ist insofern von Bedeutung, als die technische Ausführung des Skifahrens sich grundlegend unterscheidet. Sitzende Skifahrer bedienen sich eines Monoskis und zur Stabilisierung der Fahrt sogenannter Krückenski, die an den Armen befestigt werden. Stehende Skifahrer fahren entsprechend ihrer körperlichen Behinderung mit einem oder zwei Ski und benutzen entweder gewöhnliche Skistöcke oder ebenfalls Krückenski. 
Auf Grund der Heterogenität und eines kleinen Probandenkollektivs ist die Arbeit als Pilotstudie zu begreifen.

Grundlage der Untersuchungen ist die Bestimmung des Parameters Laktat im Vollblut und die Beschreibung der Laktatkinetik über die Zeit: Die Laktatkonzentration wurde wegen der Struktur der Skidisziplin Riesenslalom vor dem Wettkampf bzw. Training sowie zu unterschiedlichen Zeitpunkten in der Nachbelastungsphase bestimmt. In der Vorbereitungsphase im Sommer sind Messungen während der Belastung u.a. in Form spiroergometrischer Tests vorgenommen worden; in der Gruppe der sitzenden Athleten mit Hilfe eines Handbikes auf einem Laufband, die stehenden Sportler unterzogen sich Fahrradergometertests.

Insgesamt liegen vier Messreihen aus dem Wettkampf, zwei aus dem Training und drei aus der jeweiligen Vorbereitungsphase im vorangegangenen Sommer vor.

Grundsätzlich stehen in leistungsdiagnostischen Untersuchungen verschiedene Parameter zur Verfügung. Bei spiroergometrischen Untersuchungen im Labor beispielsweise werden häufig als objektive Parameter Laktat, Herzfrequenz und maximale Sauerstoffaufnahme gemessen. Auch subjektive Bewertungskriterien der Belastung seitens der Probanden (z.B. Verwendung der Borg-Skala) fließen ein.

Bei sportartspezifischen Untersuchungen im Feldversuch am Skihang steht die Durchführbarkeit im Vordergrund. In einer Wettkampfsituation ist die Untersuchung der maximalen Sauerstoffaufnahme eher schwierig. Die Messung der Laktatkonzentration vor und nach Belastung hingegen erscheint praktikabel; „Laktat ist auch unter Feldbedingungen ein einfach zu messender Parameter“ (de Marées 2002, S. 474). Die Laktatbestimmung kann anhand kapillären Blutes aus dem Ohrläppchen rasch und einfach erfolgen; häufige Wiederholungen dieser Untersuchung sind wegen nur geringer benötigter Blutmengen möglich. Somit erfüllt sie die Kriterien für einen ökonomischen Test (Vgl. Weineck 2007, S.77). Weiterhin denkbar wäre als Beurteilungskriterium sportlicher Leistung die Aufzeichnung der Herzfrequenz vor, während und nach Belastung.

Im Feldversuch zu berücksichtigen sind variable Faktoren gegenüber Untersuchungen unter Laborbedingungen: Schneebeschaffenheit, Steigung des Hanges, Wind, Temperatur, Höhe und Material (Vgl. Fecht 1980) können Auswirkungen haben. 
Die Laktatkonzentration ist abhängig von vielen Faktoren: Zunächst Laktatproduktion, -umverteilung und -elimination. Doch auch äußere Faktoren beeinflussen sie: Vorbelastung, Ernährung oder die Messmethode (Vgl. auch Heck 1990, S. 191-193).

In der Studie haben alle Athleten eine vergleichbare Vorbelastung. In allen Jahren sind am Vortag des Wettkampfs Trainingseinheiten erfolgt (inkl. Trainingsmessungen in Jahr 1 und 2), und der Riesenslalom wurde als erste Disziplin ausgetragen.

Die Messungen erfolgten aus Kapillarblut aus dem Ohrläppchen des Probanden, als Messgerät fungierte in allen Messreihen das Gerät „Lactate Scout“ der Firma SensLab. Um Temperaturschwankungen und eventuelle Fehlbestimmungen $\mathrm{zu}$ vermeiden, ist das Gerät unter der Kleidung möglichst nah am Körper getragen worden. Die Probenentnahmen wurden stets durch denselben Untersucher vorgenommen.

Auf Grund der großen Heterogenität und der differierenden Skitechnik wurden die erhobenen Daten zunächst innerhalb der Gruppen Sitzend und Stehend einzeln betrachtet. Die Statistik ist dementsprechend v.a. deskriptiv; bei einem anschließenden Vergleich der Gruppen untereinander und mit einem gesunden Probandenkollektiv wurden Varianzanalysen durchgeführt. Dies geschah vorrangig zur Feststellung von Trends, die statistische Aussagekraft erscheint bei einem so kleinen und heterogenen Probandenkollektiv eingeschränkt.

\subsection{Diskussion der Ergebnisse}

\subsubsection{Laktat als leistungsdiagnostischer Parameter}

In leistungsdiagnostischen Untersuchungen werden verschiedene Parameter bestimmt und ausgewertet, um Rückschlüsse auf die Leistungsfähigkeit eines Sportlers ziehen zu können. Resultierend aus den Ergebnissen werden Trainingsplanungen vorgenommen bzw. bestehende verändert. Ein möglicher Parameter ist - wie in der vorliegenden Studie - die Laktatkonzentration im Vollblut.

Laktat wird im Organismus im Rahmen der Glykolyse produziert; in Abhängigkeit von einer Belastungsform - aerob oder anaerob - kann es zu Pyruvat oxidiert werden oder fällt als Endprodukt der anaeroben Glykolyse an. Je nach Belastungsintensität und -dauer fällt zunächst vermehrt Laktat im Muskel an und wird anschließend ins Blut transportiert (Vgl. Abb.1). Nach Beenden einer physischen Leistung fällt die Konzentration ab. 
Die Produktions-, Verteilungs- und Eliminationsprozesse verlaufen parallel und versetzt. Der Organismus versucht während dieser Prozesse eine Homöostase zu schaffen.

Der Verlauf der Laktatkonzentration während oder nach Belastung wird häufig anhand von Laktatkonzentrationskurven dargestellt. Der Ausgangswert, Maximalwerte, Steigung der Kurve und Verhalten des Laktats nach Belastungsende sind beurteilbar.

Leistungsdiagnostische Untersuchungen sollten auf die Struktur einer Sportart - in diesem Fall die des Skisports - Bezug nehmen.

In der Sportart Ski alpin stehen vor allem Schnelligkeit, Ausdauer, Reaktionsvermögen, technische Fertigkeiten und die neuromuskuläre Koordination im Vordergrund. Die Elemente sind in den Skidisziplinen unterschiedlich gewichtet, z.B. die Bewegungselemente: während im Super-G oder der Abfahrt mehr statische Arbeit geleistet wird, ,werden im Slalom und Riesenslalom mehr Bewegungszyklen absolviert" (Temme 2001, S. 37). Weiterhin ist im Skisport generell die hohe Stressbelastung von Bedeutung (Vgl. Kindermann und Keul 1977a, S. 83), infolge erhöhter Katecholaminsekretion wird die Laktatproduktion durch Aktivierung der anaeroben Glykolyse noch unterstützt (Vgl. Kindermann und Keul 1977b, S.74).

Im Riesenslalom (Rennlauf) ist eine vorwiegend anaerobe Energiebereitstellung zu erwarten. Folglich ist anzunehmen, dass die Laktatkonzentration im Blut während und nach Belastung merklich ansteigen wird. Die Analyse des Parameters Laktat ist im Riesenslalom vor und nach Belastung möglich; der Verlauf der Laktatkonzentration sowie Maximalwerte können beschrieben werden. Auf Grund rascher und einfacher Analysemöglichkeiten ist diese Messung unter Feldbedingungen gut geeignet.

\subsubsection{Vergleich sitzende und stehende Sportler - Wettkampf und Training}

Die Auswertung der Ergebnisse erfolgte zunächst in Form von Einzelfallanalysen der sitzenden und stehenden Sportler.

Im Winter wurde jeweils vor dem Wettkampf und dem Trainingslauf ein Ruhewert bestimmt; die Messzeitpunkte in der Nachbelastungsphase sind unmittelbar nach Ende der Belastung, nach drei, fünf und sieben Minuten angesiedelt. In Jahr 2 liegen keine Werte zum Zeitpunkt R4 vor. Im Training wurde jeweils bis fünf Minuten nach Ende des Laufs gemessen (T3). 
In der Gruppe der sitzenden Sportler (insgesamt sechs Athleten) liegen die Ruhewerte in den Jahren im Wettkampf im Mittel zwischen 1,87 und 2,3 mmol/l, die Maximalwerte sind weit gestreut zwischen 2,9 und 6,9 mmol/l. Die im Training erreichten Werte sind insgesamt niedriger. Der Verlauf der Laktatkonzentrationskurve ähnelt sich bei den Probanden, jedoch nicht das Niveau der Laktatspiegel. Zumeist nähert sich die Kurve noch während des Messzeitraums dem Ruhewert an. Sportler 1 hat über alle Jahre betrachtet die niedrigsten Laktatkonzentrationen nach Belastung.

In der Gruppe der stehenden Sportler haben insgesamt acht Athleten an den Messungen teilgenommen. Auch sie weisen eine große Streuung der Laktatkonzentration in der Nachbelastungsphase auf. Bereits die Ruhewerte variieren stark, die Maximalwerte betragen vereinzelt über $10 \mathrm{mmol} / \mathrm{l}$. Die gemessenen Laktatkonzentrationen sind höher als bei den sitzenden Sportlern. Im Gegensatz zu anderen Probanden, die zum Teil einen verzögerten Rückgang oder gar erneute Anstiege des Laktats in der Phase nach Ende des Laufs zeigen, ist beispielsweise bei Sportler 4 noch im Messzeitraum ein deutlicher Abfall des Spiegels zu verzeichnen.

Die Unterschiede in der Höhe der Laktatkonzentrationen könnten durch die Heterogenität der gesamten Gruppe, aber auch der einzelnen Kollektive begründet sein.

Aigner et al. führten leistungsdiagnostische Untersuchungen mit Rollstuhlfahrern durch. Innerhalb der Probandengruppe wurden Untergruppen entsprechend der verschieden lokalisierten Querschnittlähmungen gebildet. Sie konnten nachweisen, dass die Laktatwerte umso höher waren, je niedriger der Querschnitt lokalisiert war und demzufolge mehr aktive Muskeln zur Bewegungsausführung eingesetzt werden konnten (Vgl. Aigner et al. 1990, S. 313 f.).

Sitzende Sportler fahren mit einem Monoski und verwenden zur Stabilisierung Krückenski, die an den Armen fixiert werden. Primär werden die Muskelgruppen des Rumpfes, der Schultern und Arme benutzt. Das Ausmaß variiert in Abhängigkeit von den körperlichen Behinderungen. Einige Athleten haben eine tief lokalisierte Querschnittlähmung, so dass sie die Bauchmuskeln und Teile der Hüftmuskulatur zur Bewegungsausführung einsetzen können. Andere wiederum - mit einem beispielsweise in Höhe des Thorakalmarks lokalisierten Querschnitt - setzen vermehrt die Muskeln von Armen und Schultern zur Stabilisierung des Rumpfes ein. 
Laktat wird unter Belastung in der Muskulatur produziert und gelangt anschließend ins Blut. Ist die Muskelmasse größer, bedeutet es, dass der Produktions- aber auch Eliminationsort für Laktat größer ist. Dies könnte eine höhere Laktatkonzentration im Blut erklären.

Auch für die Unterschiede innerhalb der Gruppe Stehend kann dieses Argument Verwendung finden: Die funktionelle Muskelmasse eines Sportlers, der beide Beine zum Skifahren einsetzen kann, ist größer als die eines einseitig oberschenkelamputieren Athleten. Höhere Laktatkonzentrationen im Blut wären die Folge.

In der Einzelfallbetrachtung fällt auf, dass bei vielen sitzenden Athleten geringere Laktatkonzentrationen im Blut nachgewiesen werden können. Eine exemplarische Gegenüberstellung zweier Sportler unterschiedlicher Gruppen (Sportler1 und 4) zeigt bereits Tendenzen auf, die sich im Gesamtvergleich der Gruppen bestätigen. Sportler 4 erreicht deutlich höhere Laktatkonzentrationen, die nach Belastung abfallen, jedoch innerhalb des Messzeitraums nicht Ruhewertniveau erreichen. Sportler 1 hingegen zeigt einen verhalteneren Anstieg der Laktatkonzentration und einen Rückgang in Bereiche von R0. Im Training ist ein ähnliches Verhalten zu beobachten, jedoch differieren die Werte von Rennen und Training bei Sportler 4 deutlich, während Sportler 1 sich ungefähr auf einem Niveau bewegt.

Im Gesamtvergleich der Gruppen Sitzend und Stehend, in den alle Einzelwerte der Athleten aus Rennen und Training eingeflossen sind, kann gezeigt werden, dass die Laktatkonzentrationen der sitzenden Probanden signifikant niedriger als jene der stehenden sind. Die Trainingswerte aller stehenden Athleten sind niedriger angesiedelt als die im Rennen erhobenen.

Die Streubreite der Werte ist groß, die Einzelwerte der sitzenden und stehenden Athleten im Rennen überlappen kaum, im Training kommt es hingegen vermehrt zu Überschneidungen.

Hinsichtlich des Verlaufs der Laktatkonzentration über die Zeit kann kein signifikanter Zusammenhang nachgewiesen werden.

In der Varianzanalyse der Daten aus dem Training bilden sich andere Zusammenhänge ab als in denen aus dem Rennen und der Gesamtauswertung. Zwischen sitzenden und stehenden Athleten zeigen sich keine signifikanten Unterschiede der Laktatkonzentrationen. Insgesamt ist die Streuung der Einzelwerte deutlich größer als im Rennen. Dies könnte durch eine zu geringe Zahl an Messwerten begründet werden. 
Als Ursache der insgesamt niedrigeren Trainingsergebnisse ist zum einen die geringere Distanz des Trainingsrennens im Vergleich zum Rennlauf zu vermuten, die Belastungszeit ist kürzer und damit die -intensität geringer. Zum anderen fehlt im Training die Stressbelastung. In einer Wettkampfsituation werden vermehrt Katecholamine ausgeschüttet; dies wirkt sich wiederum in einem Anstieg der Herzfrequenz sowie einer gesteigerten Glykolyse und daraus resultierend einer Erhöhung der Laktatkonzentration im Blut aus (Kindermann und Keul 1977b, S.74). Geringere Laktatkonzentrationen wären somit zu erklären.

Die Unterschiede der Laktatkonzentrationen zwischen sitzenden und stehenden Sportlern könnten auf die anders geartete Skitechnik und Belastung zurückzuführen sein. Des Weiteren ist der Anteil an funktioneller Muskelmasse unterschiedlich. Als Beispiel seien Sportler 1 und 4 angeführt: Sportler 1 rekrutiert v.a. die Muskulatur von Rumpf und Armen, Sportler 4 benutzt die Bein-, Hüft- und Rumpfmuskulatur zum Skifahren. Letzterer hat also mehr Muskelmasse zur Verfügung, um Laktat zu produzieren und abzubauen. Somit sind höhere Laktatkonzentrationen in oder nach einer Belastungssituation zu erwarten.

Der Gesamtvergleich von sitzenden und stehenden Probanden ist mit Vorsicht zu bewerten. Die Kollektive sind in sich sehr heterogen, so dass bereits Vergleiche innerhalb einer Gruppe schwierig sind. Weiterhin ist die gesamte Probandenanzahl gering.

Durch die Gegenüberstellung können deutliche Unterschiede in der Höhe der Laktatkonzentration nachgewiesen werden; die verschieden hohen Messwerte zeigen sich sowohl im Mittelwertvergleich als auch bei einer vergleichenden Betrachtung der Einzelfallanalysen der jeweiligen Jahre. Dies ist als Trend zu verstehen. Um die Ergebnisse verifizieren zu können, wäre ein größeres Kollektiv wünschenswert.

Die Ergebnisse der Messreihen sind im Weiteren dahingehend untersucht worden, ob genannte Unterschiede innerhalb der Gruppen auch auf die Leistungsstärke der Sportler zurückzuführen sein könnten.

Im Hinblick darauf wurden leistungsstärkere und -schwächere Athleten innerhalb der Gruppen miteinander verglichen. 


\subsubsection{Vergleich erfolgreiche und weniger erfolgreiche Sportler}

Die Laktatkinetik verändert sich in Abhängigkeit vom Training. Kindermann und Keul konnten bereits zeigen, dass Trainierte eine bessere Toleranz hinsichtlich einer höheren Laktatazidose aufweisen. Weiterhin ist die Laktatkonzentration bei Untrainierten höher; Trainierte erreichen erst zu einem späteren Zeitpunkt eine kritische Laktatzunahme, zudem wird die Muskulatur ökonomischer eingesetzt (Vgl. Kindermann und Keul 1977b, S. 82; Klinke und Silbernagl 2003, S. 523). Training führt also grundsätzlich zu metabolischen Veränderungen.

Im Vergleich von erfolgreichen und weniger erfolgreichen Athleten wurde erfolgreich definiert als Platzierung im Wettkampf innerhalb der ersten zehn Plätze, weniger erfolgreich als Platzierung ab dem elften Rang. Die Einzelwerte der jeweiligen Athleten wurden berücksichtigt.

Die erfolgreichen Sportler 1 und 4 weisen gegenüber den weniger erfolgreichen (14 und 5) niedrigere Laktatwerte, außerdem einen raschen Anstieg und einen kontinuierlichen Abfall der Laktatkonzentration im Blut in der Nachbelastungsphase bereits während des Messzeitraums, z.T. sogar auf Ruhewertniveau (sitzender Athlet 1), auf. Eine gute Regenerationsfähigkeit, die im Riesenslalom wegen zweier Renndurchläufe von großer Bedeutung ist, kann angenommen werden.

Im Gesamtvergleich zeigt sich folgende Anordnung hinsichtlich der Höhe der Laktatkonzentrationen im Blut:

Erfolgreich sitzend $<$ weniger erfolgreich sitzend $<$ erfolgreich stehend $<$ weniger erfolgreich stehend.

Der bereits nachgewiesene signifikante Unterschied der Laktatspiegel von sitzenden und stehenden Athleten bestätigt sich; ein statistisch signifikanter Zusammenhang mit der Leistungsstärke ist nicht nachweisbar.

Erfolgreiche Sportler zeigen eine bessere Regenerationsfähigkeit und geringere Laktatkonzentrationen, im Umkehrschluss wäre bei Vorliegen dieser Konstellation eine bessere Leistungsfähigkeit anzunehmen. Die Zahl der verglichenen Sportler ist sehr gering, so dass - auch wenn sich die Tendenzen eines Zusammenhangs von Laktatkinetik und Leistungsstärke in den Einzelfallbetrachtungen zeigen - nicht zu erwarten ist, dass sie sich auch statistisch bestätigen ließen. 


\subsubsection{Feldversuch versus unspezifische Messungen in der Vorbereitungsphase im Sommer}

Das Hauptaugenmerk in dieser Arbeit liegt auf sportartspezifischen Untersuchungen am Skihang.

Darüber hinaus sind in der Vorbereitungsphase der jeweiligen Jahre Untersuchungen unter standardisierten und reproduzierbaren Bedingungen im Labor durchgeführt worden. Diese Tests waren anders strukturiert:

Sitzende Sportler führten spiroergometrische Untersuchungen auf einem Handbike durch, das auf einem Laufband gesteuert wurde. Der Antrieb erfolgte über Drehkurbeln mit Hilfe der Arme. Unter anderem wurden die Herzfrequenz und Laktat im Blut in der Nachbelastungsphase untersucht. Stehende Sportler absolvierten die Tests auf einem Fahrradergometer; die Laktatbestimmung erfolgte v.a. während der Belastung, vereinzelt wurden Nachbelastungswerte erhoben. Aus diesen Untersuchungen sind vorwiegend Aussagen über die Ausdauerleistungsfähigkeit abzuleiten.

In der Gruppe der sitzenden Athleten wurden in den Sommermessungen deutlich höhere Laktatkonzentrationen im Blut ermittelt als im Winter. Bei dem erfolgreichen Sportler 1 zeigt sich ein Rückgang des Laktatspiegels, jedoch nicht bis auf Ruhewertniveau. Der Verlauf deutet insgesamt auf eine gute Regenerationsfähigkeit hin.

Die Laktatwerte der stehenden Sportler liegen ebenfalls deutlich über Wettkampf- und Trainingsniveau. Bei einigen Athleten sind Nachbelastungswerte bestimmt worden. In der Messung während der Belastung ist insbesondere Sportler 4 mit einer Leistung von 425 Watt hervorzuheben; insgesamt weisen aber alle Athleten entsprechend ihrer Konstitution eine gute bis sehr gute Grundlagenausdauer auf.

Die Sommermessungen im Gesamten betrachtet werden also in beiden Probandengruppen deutlich höhere Laktatkonzentrationen erreicht als unter sportartspezifischen Bedingungen. Ein bedeutender Unterschied besteht in der Struktur der leistungsdiagnostischen Tests. Unter sportartunspezifischen Bedingungen, beispielsweise auf einem Fahrradergometer, wird die Belastung stufenweise gesteigert und dauert länger an. Im Feldversuch am Skihang ist die Belastung kurz und die -intensität annähernd gleichbleibend. Ein Vergleich wird dadurch erschwert.

Die Zielsetzungen von sportartunspezifischen und sportartspezifischen leistungsdiagnostischen Untersuchungen unterscheiden sich: Grundsätzlich ist aus den 
Sommermessungen, die unter sportartunspezifischen Bedingungen und während der Belastung durchgeführt werden, eine Aussage über die Grundlagenausdauer der Athleten abzuleiten; die Tests sind in vielen Sportarten in gleicher Weise strukturiert.

Sportartspezifische Untersuchungen am Skihang in Form von Messungen in der Nachbelastungsphase hingegen dienen der Interpretation der Regenerationsfähigkeit eines Athleten.

Im Labor würde über eine Ausdehnung des Untersuchungszeitraums in die Nachbelastungsphase, wie bei den meisten sitzenden Sportlern, auch unter standardisierten Bedingungen eine Beurteilung der Regenerationsfähigkeit ermöglicht; ergänzende umfangreichere Messungen in dieser Phase wären also wünschenswert - sowohl im Hinblick auf einen Vergleich der Laktatkinetik in Sommer und Winter (sportartunspezifisch und sportartspezifisch) als auch einen Vergleich der Sportler.

\subsubsection{Vergleich Behindertensportler und gesunde Probandengruppe}

Die maximale Laktatkonzentration des gesamten Probandenkollektivs in den Messungen im Feldversuch liegt bei 10,7 mmol/l (Sportler 5, Jahr 1, R3) im Winter (Rennen). Der Trend unterschiedlich hoher Laktatkonzentrationen im Vergleich sitzender und stehender Sportler konnte gezeigt werden.

Sportartspezifische Untersuchungen existieren im Behindertensport Ski alpin bisher nicht, so dass keine Vergleiche der Ergebnisse mit anderen Studien angestellt werden können.

Im Nichtversehrtensport hingegen liegen einige Untersuchungsreihen vor (Vgl. Kapitel 1.5). Festzuhalten ist zunächst, dass die Laktatkonzentration abhängig vom Können eines Skifahrers und von der Belastungsform ist.

In den im Freizeitsportbereich durchgeführten leistungsdiagnostischen Tests wurden ebenfalls Laktatspiegel im Blut in der Nachbelastungsphase bestimmt. Es wurden eher niedrige Laktatkonzentrationen beobachtet. Der Versuchsaufbau der einzelnen Studien war anders strukturiert: nach Ende der Belastung wurde nur zu einem Zeitpunkt der Laktatwert im Blut bestimmt; im Hinblick auf die Laktatkinetik wurden keine Aussagen getroffen. Des Weiteren ist das Leistungsniveau der Probanden nicht vergleichbar mit dem der in dieser Studie untersuchten Sportler.

Diese Voraussetzungen sind eher in den Versuchsreihen aus dem Leistungssportbereich erfüllt. 
Lochner et al. (1983) untersuchten Skilehrer im Riesenslalom; die Belastungsdauer und -intensität entspräche also eher den behinderten Sportlern, dies gilt jedoch nicht für das Leistungsniveau.

Veicsteinas et al. (1984) und Saibene et al. (1985) führten Laktatmessungen im Rahmen konstruierter Riesenslalomrennen durch, die Probanden bewegten sich im Leistungssportbereich. Bei beiden Studien ist zu berücksichtigen, dass die Daten nicht unter Wettkampfbedingungen erhoben wurden, so dass die Stressbelastung als geringer einzustufen sein dürfte. Die Beurteilung des weiteren Verlaufs der Laktatkonzentration in der Nachbelastungsphase ist wegen der Bestimmung nur eines Wertes nicht möglich.

Mayr (2008) ermittelte während eines Freeride-Wettkampfes Laktatkonzentrationen im Blut von Probanden internationalen Niveaus, so dass eine entsprechende Stressbelastung anzunehmen ist; die Bewegungsstruktur der Disziplinen Freeride und Riesenslalom differiert jedoch. Des Weiteren wurde - wie bei den anderen Studien - nur eine Messung in der Nachbelastungsphase durchgeführt, so dass sich diese Versuchsreihe nicht für einen Vergleich mit den Ergebnissen der behinderten Sportler eignet.

Hintzy et al. (2009) untersuchten jugendliche Rennläufer im Riesenslalom; Leistungsniveau und Belastungsintensität wären vergleichbar. Jedoch ist zu berücksichtigen, dass Jugendliche eine andere Zusammensetzung der Skelettmuskulatur aufweisen und sich somit Laktatproduktion, -verteilung und -elimination von der Erwachsener unterscheiden (Vgl. Hintzy et al. 2009, S. 500). Kindermann und Keul nehmen Bezug auf Eriksson et al., die Unterschiede hinsichtlich der Höhe der Laktatkonzentration bei Kindern und Erwachsenen beschrieben; so gingen die niedrigeren Blutlaktatkonzentrationen im Kindesalter mit einer verminderten Produktion in der Muskulatur einher, weiterhin nehme die anaerobe Kapazität mit der Entwicklung zu (Vgl. Kindermann und Keul 1977b, S. 80).

Die größte Übereinstimmung von Versuchsbedingungen und -aufbau findet sich in der Studie von Temme (2001). Sie führte ebenfalls Laktatmessungen zu mehreren Zeitpunkten in der Nachbelastungsphase eines Wettkampfs durch, so dass sowohl Laktatkonzentration als auch die Kinetik beschrieben werden konnten. Weiterhin bewegen sich die Sportler auf einem ähnlichen Leistungsniveau.

Sie bestimmte im Feldversuch u.a. im Riesenslalom neben anderen Parametern die Laktatkonzentration im Blut vor und bis 30 Minuten nach Belastung. In der Gruppe der Frauen betrug der Maximalwert 13,8 mmol/l Blut (Mittelwerte zwischen 9,6 bis $12 \mathrm{mmol} / \mathrm{l}$ ), bei den Männern stiegen die Werte zum Teil bis über $20 \mathrm{mmol} / \mathrm{l}$ an; insgesamt lagen sie im 
Mittel zwischen 14-20 mmol/l. Ähnlich hohe Laktatkonzentrationen beschrieben Raas und Hörtnagel (1984) sowie Zintl und Gebauer (1985) mit 15,2 $\pm 2,1$ mmol/l bzw. 10-11 mmol/l (Vgl. Röder 2001, S. 13).

Eine Vergleichbarkeit mit den Probanden der vorliegenden Studie ist in der körperlichen Unversehrtheit nicht gegeben, jedoch im Hinblick auf das Leistungsniveau.

Zum Vergleich der Behindertensportler und der gesunden Probandengruppe von Temme sind verschiedene Kollektive gebildet worden:

Sitzend, Stehend, gesunde Frauen und gesunde Männer.

Aus den jeweiligen Einzelwerten erfolgte die Bestimmung eines Mittelwertes für jede Gruppe. Zum Zweck einer besseren Vergleichbarkeit zwischen behinderten und gesunden Sportlern wurden die Messzeitpunkte aus der Arbeit von Temme extrapoliert.

Das Niveau der Laktatkonzentrationen der gesunden Probandengruppe liegt bei einem Vergleich mit den sitzenden und stehenden Athleten aus dem Behindertensportbereich signifikant höher. Hinsichtlich des Verlaufs über die Zeit zeichnet sich ein signifikanter Zusammenhang ab.

Insgesamt zeigt sich folgende Anordnung hinsichtlich der Höhe der Laktatkonzentrationen im Blut:

Sitzend $<$ Stehend $<$ Gesunde Frauen $<$ Gesunde Männer.

Temme begründet den Unterschied zwischen Frauen und Männern u.a. mit der geringeren Menge der aktiven Muskelmasse ersterer (Vgl. Temme 2001, S. 35).

In der vorliegenden Studie wird keine weitere Unterteilung der Probanden in Frauen und Männer vorgenommen. Das Kollektiv ist heterogen, alle Sportler weisen andere körperliche Behinderungen auf. Eine Einzelfallbetrachtung erfolgt ohnehin.

Der maximal gemessene Wert in der Gruppe der Behindertensportler (10,7 mmol/l) wird von einem stehenden Probanden erreicht. Die Menge der aktiven Muskelmasse als Begründung für Unterschiede in der Höhe der Laktatkonzentration ist bereits beim Vergleich der einzelnen Kollektive dargelegt worden. Auch hier ist die beschriebene Diskrepanz der Spiegel entsprechend zu begründen.

Die sitzenden Athleten einmal - auf Grund der differierenden Technik - ausgeklammert, ist trotzdem ein signifikanter Unterschied der Laktatwerte zwischen stehenden Sportlern aus dem Behindertensportbereich und beiden Gruppen aus der gesunden Vergleichsgruppe gegeben. 
Ein weiteres Argument für verschieden hohe Laktatwerte könnte eine durch die Behinderung veränderte Statik sowie Bewegungsausführung und dadurch bedingt defensivere Fahrweise sein. Die Belastung gestaltet sich anders, die Laktatkonzentration könnte niedriger sein.

Beim Vergleich von Temme und der vorliegenden Studie wurden insgesamt nur wenige Probanden gegenübergestellt. Die Streubreite innerhalb der einzelnen Gruppen ist hoch. Die nachgewiesenen Signifikanzen sind mit Vorsicht zu bewerten; sie sind eher als Trends zu begreifen.

\subsubsection{Laktatkonzentrationskurven in der Nachbelastungsphase}

Zur Darstellung und Auswertung der Laktatkinetik über die Zeit eignen sich Laktatkonzentrationskurven. In der Literatur werden verschiedene Möglichkeiten der Darstellung angegeben. Grundsätzlich zu unterscheiden sind Kurven, die den Laktatverlauf während einer Belastung abbilden von Kurven aus der Nachbelastungsphase.

Einen Überblick darüber liefern Koch und Raschka: Wachstumskurven beispielsweise eignen sich insbesondere für die Darstellung des Laktatverlaufs während einer andauernden Belastung (Vgl. Koch und Raschka 1999). Bereits durch Training oder Ernährung ist eine Verschiebung der Kurven möglich. Letztlich dienen sie der Beurteilung der Grundlagenausdauer und des Leistungsstandes eines Sportlers (Vgl. Braumann et al. 1987, S. 37).

Die Interpretation von Laktatkonzentrationskurven in der Nachbelastungsphase dient hingegen vorrangig der Beurteilung der Regenerationsfähigkeit eines Sportlers. Laktatproduktion, Verteilung im Blut und Abbau sind Prozesse, die nebeneinander verlaufen; ein starres Modell zur Erfassung und Wiedergabe der physiologischen Prozesse scheint nicht geeignet. Eine Darstellung über Polynome oder Exponentialfunktionen wäre denkbar (Vgl. Koch und Raschka 1999).

Anhand der Ergebnisse einiger Sportler wurden die Verläufe der Laktatkonzentrationskurven der einzelnen Jahre sowie die Mittelwerte erneut dargestellt, Exponentialfunktionen und Polynome konstruiert. Weiterhin erfolgten anhand dieser Funktionen Berechnungen von Laktatwerten $\mathrm{zu}$ einem Zeitpunkt $\mathrm{x}(\mathrm{x}=4$ Minuten nach Belastungsende), $\mathrm{zu}$ dem keine Ergebnisse vorliegen.

Über das Polynom bestimmte Laktatwerte liegen bei allen Probanden oberhalb derer mithilfe der Exponentialfunktionen berechneten; weiterhin sind sie den tatsächlich gemessenen 
Werten nach drei und fünf Minuten näher. Der Verlauf der Laktatkonzentration in der Nachbelastungsphase scheint also genauer mit Hilfe von Polynomen abgebildet werden zu können.

Ziel der regelmäßigen Aufzeichnung von Laktatkonzentrationskurven ist, durch Verlaufsbeobachtungen Rückschlüsse auf die „Effizienz einer durchgeführten Trainingsperiode“ ziehen zu können (Vgl. Braumann et al. 1987, S. 38).

Beim Vergleich der Athleten fällt bei Sportler 1 und 4 (mit Ausnahme von Jahr 2) auf, dass sich die einzelnen Laktatwerte - sowohl gemessene als auch berechnete - dem Mittelwert relativ annähern. Bei Sportler 5 hingegen sind deutliche Abweichungen vom Mittelwert zu beobachten. Die Laktatkonzentrationskurven der einzelnen Jahre liegen weiter auseinander. Erstgenannte Athleten zeigen über die Jahre eine kontinuierlich gute Leistung. Die Leistungen von Sportler 5 variieren innerhalb der verschiedenen Messreihen. Es ließe sich nun vermuten, dass Sportler in einem annähernd gleichbleibend guten Trainingszustand relativ konstante Verläufe der Laktatkonzentrationskurven zeigen.

Zusammenfassend eignet sich also aus physiologischer Perspektive die Darstellung des Verlaufs der Laktatkonzentration im Blut über die Zeit nach Belastung über Polynome als Kurvenanpassungsverfahren.

Eine Besonderheit stellen die Messwerte aus dem Rennen im zweiten Jahr dar. Die errechneten Werte differieren deutlich von allen anderen. Bei Betrachtung des Polynoms fällt auf, dass es sich bei Sportler 4 kurzfristig im negativen Bereich bewegt und auch bei Sportler 1 einen Abfall nahe einer Konzentration von $0 \mathrm{mmol} / \mathrm{l}$ aufweist. Dies ist unter physiologischen Bedingungen nicht möglich, so dass es sich um einen Fehler handeln muss. In Jahr 2 wurden nur drei Messwerte in der Nachbelastungsphase bestimmt. In diesem Fall könnte fraglich sein, ob eine Darstellung der Laktatkonzentration über ein Polynom bei wenigen Werten geeignet ist. Die anhand der Exponentialfunktion berechneten Werte sind in Jahr 2 konstant. Eine Darstellung der Laktatkonzentration über Exponentialfunktionen könnte also bei nur wenigen Messwerten besser geeignet sein.

Insgesamt betrachtet sind die anhand des Polynoms berechneten Werte bei Vorliegen mehrerer Messwerte jedoch genauer.

Die Messintervalle in dieser Studie sind vergleichsweise groß. Der Zusammenhang von Leistung und Verhalten der Laktatkonzentrationskurve müsste in zahlreichen Messreihen in 
kleineren Intervallen bestätigt werden; denkbar wären regelmäßige Untersuchungen während der Trainings- und Wettkampfphase.

Diese sind v.a. in den Disziplinen Riesenslalom und Slalom sinnvoll, da eine gute Regenerationsfähigkeit wegen vieler Richtungswechsel und zweier $\mathrm{zu}$ absolvierender Renndurchläufe bedeutend ist. In Abfahrt und Super-G wird nur jeweils ein Renndurchlauf gefahren, die Läufer erholen sich anschließend.

Die Bestimmung der Laktatspiegel in der Nachbelastungsphase wäre im Bereich des Behindertenskisports also gerade in Slalom und Riesenslalom in der sportartspezifischen Diagnostik ein adäquates Mittel zur Trainingssteuerung.

\subsection{Ausblick}

Es hat sich gezeigt, dass eine sportartspezifische Leistungsdiagnostik im Behindertensport Ski alpin grundsätzlich praktikabel ist. Laktatmessungen eignen sich zur Interpretation der Leistungsfähigkeit und Regenerationsfähigkeit eines Skifahrers.

Im Skisport, in dieser Untersuchung anhand des Riesenslaloms betrachtet, ist eine gute Regeneration von großer Bedeutung - v.a. in den Disziplinen Slalom und Riesenslalom, in denen zwei Renndurchläufe in kürzester Zeit zu absolvieren sind. Durch bisherige sportartunspezifische leistungsdiagnostische Untersuchungen in der Vorbereitungsphase sind Aussagen hinsichtlich der Ausdauerleistungsfähigkeit eines Athleten möglich, die in der Trainingsvorbereitung bedeutend ist. Die Ergänzung durch sportartspezifische Leistungsdiagnostik verfolgt das Ziel, unter Wettkampfbedingungen bzw. unter Simulation der Biomechanik der Sportart Aussagen zur Leistungsfähigkeit und Regenerationsfähigkeit treffen zu können.

Die Zusammensetzung des Probandenkollektivs dieser Studie spiegelt auch die Heterogenität einer Mannschaft im Behindertensport wider. Jede körperliche Behinderung bedeutet unterschiedliche Bewegungsmöglichkeiten. Aus den Laktatmessungen ist zu erkennen, wie unterschiedlich das Niveau der Laktatkonzentration auch innerhalb eines hinsichtlich der Leitungsstärke (Leistungssportler) ähnlichen Kollektivs sein kann. Dies zeigt sich sowohl innerhalb der einzelnen Gruppen als auch im Vergleich der Gruppen Sitzend und Stehend. Eine Vergleichbarkeit der Leistungen untereinander ist erschwert. 
Laktatkonzentrationskurven in der Nachbelastungsphase erlauben eine Beurteilung der Regenerationsfähigkeit eines Athleten. Eine gemittelte Laktatkonzentrationskurve aller Sportler, zu der einzelne in Vergleich gesetzt werden können, ist aus eben genannten Gründen nicht denkbar.

Regelmäßige Erhebungen solcher Kurven bei jedem einzelnen Sportler - auch Messungen nach beiden Renndurchläufen - würden die Beurteilung des aktuellen Leistungsstandes im Sinne einer Verlaufsbeobachtung unterstützen. So könnte gezielt auf den Trainingsprozess Einfluss genommen werden. Die in der Studie durchgeführten Untersuchungen haben gezeigt, dass die Interpretation der Laktatkonzentrationskurven im Riesenslalom Erkenntnisse bezüglich des Trainingsprozesses liefern kann. Interessant wäre weiterhin zu untersuchen, ob dies ebenso für die anderen Skidisziplinen, v.a. für den Slalom, anwendbar ist.

Zusammengefasst bedeutet dies, dass regelmäßige Untersuchungen am Skihang gerade im Behindertenskisport wünschenswert wären, um eine noch gezieltere Trainingssteuerung vornehmen zu können. Eine Erweiterung der Untersuchungen am Hang durch andere leistungsdiagnostische Parameter, v.a. der Herzfrequenz, ist anzustreben, um differenziertere Ergebnisse zu erhalten; und gerade die Herzfrequenzableitung ist eine unter Feldbedingungen einfach durchzuführende Untersuchung. Auch wären ergänzende Untersuchungen in den anderen Skidisziplinen wünschenswert.

Unterstützend sollten weiterhin die Untersuchungen im Labor erfolgen. Die zusätzliche Erhebung von Nachbelastungswerten, v.a. bei den stehenden Athleten, würde weitere vergleichbare Ergebnisse liefern können.

Im Skisport besteht eine große Besonderheit des Trainingsprozesses darin, dass eine sportartspezifische Vorbereitung gerade im Sommer schwierig ist. Trainingsmöglichkeiten bestehen in Gletscherskigebieten oder auch seit einigen Jahren in Skihallen.

Diese Möglichkeit sollte auch zur Durchführung leistungsdiagnostischer Untersuchungen genutzt werden. Die Bedingungen in einer Skihalle sind relativ stabil. Umgebungs- und Schneetemperatur variieren kaum. Zudem kann dasselbe Übungsgelände genutzt werden. Die Durchführung von Tests wäre also ohne großen Aufwand möglich und somit die Gewinnung von unmittelbaren Vergleichsdaten während der Ausübung der Sportart für Wintermessungen am Skihang. 
Seit Kurzem werden Untersuchungen im Windkanal durchgeführt (Vgl. http://www.dlr.de.Home). Gerade für Monoskifahrer, die während einer Abfahrt durch ihre sitzende Position großen Luftwiderständen ausgesetzt sind, ist eine optimale Körperposition von großer Bedeutung. Die Experimente, bei denen u.a. Videoanalysen vorgenommen werden, zielen darauf ab, Material und Körperposition zu optimieren. Diese Untersuchungen sollen bisherige leistungsdiagnostische Tests wie jene in der Studie durchgeführten im Hinblick auf eine Trainingssteuerung ergänzen.

Sowohl im Training in der Skihalle als auch im Winter am Skihang ist der Einsatz von Videoaufzeichnungen denkbar, um die Umsetzung der aus den Untersuchungen im Windkanal gewonnenen Ergebnisse in der Praxis zu überprüfen.

Wünschenswert wären insgesamt Untersuchungen mit einem größeren Probandenkollektiv. Dies birgt zwei Schwierigkeiten in sich: Zum einen wird trotzdem eine große Heterogenität der Gruppe bestehen und damit eine eingeschränkte Vergleichbarkeit bleiben. Zum anderen sind die Mannschaften im Behindertensport weniger personenstark als im Nichtversehrtensport. 


\section{Zusammenfassung}

Das Thema der vorliegenden Arbeit ist eine trainingsbegleitende Leistungsdiagnostik im Behindertensport Ski alpin.

Als Probanden der Studie fungierten Sportler aus dem Leistungssportbereich; auf Grund eines breiten Spektrums körperlicher Behinderungen und einer daraus resultierenden Heterogenität der Probandengruppe erfolgte eine Unterteilung der Athleten in die Gruppen Sitzend und Stehend.

Die Sportler sind über drei aufeinanderfolgende Jahre in Training und Wettkampf (Riesenslalom) sowie der zugehörigen Vorbereitungsphase im Sommer begleitet worden. Als leistungsdiagnostischer Parameter diente die Laktatkonzentration im Vollblut vor und zu verschiedenen Zeitpunkten nach Belastung im Verlauf. Die Entnahme erfolgte über Kapillarblut aus dem Ohrläppchen, die Auswertung anhand des Geräts Lactate Scout.

Die gewonnenen Daten wurden wegen der erwähnten Heterogenität der Probandengruppe in Form einer Einzelfallbetrachtung ausgewertet; anschließend erfolgten Vergleiche zwischen den Sportlern innerhalb einer Untergruppe sowie zwischen den Kollektiven Sitzend und Stehend.

Innerhalb der Gruppen wurden unterschiedlich hohe Laktatkonzentrationen erreicht, auch der Verlauf der Spiegel in der Nachbelastungsphase differierte. Dies ist zum einen damit zu begründen, dass der Anteil der funktionellen Muskelmasse in Abhängigkeit von der physischen Behinderung variiert. Somit ist eine geringere Menge an produziertem Laktat anzunehmen. Ein weiteres Argument könnte die Leistungsstärke der Athleten sein. Sportler, die einen guten Trainingszustand aufweisen, haben niedrigere Laktatwerte, und auch andere metabolische Veränderungen sind nachweisbar. Bei einer Gegenüberstellung einzelner erfolgreicher und weniger erfolgreicher Sportler konnten Unterschiede in der Laktatkonzentration beschrieben werden, ein statistisch signifikanter Unterschied bestätigte sich jedoch nicht.

Ein bedeutender Unterschied zwischen Laktatspiegeln von sitzenden und stehenden Sportlern konnte hingegen beschrieben werden. Im Vergleich zeigten die sitzenden Sportler signifikant niedrigere Laktatwerte als die stehenden Athleten; auf Grund des kleinen und heterogenen Probandenkollektivs wird dies jedoch als Trend begriffen. Diese Ergebnisse sind ebenfalls auf die Diskrepanz der aktiven Muskulatur zurückzuführen.

Ein abschließender Vergleich der Daten erfolgte mit einer Studie, die Temme (2001) an Leistungssportlern durchführte. Da die Untersuchungen sowohl während des Wettkampfs durchgeführt wurden, als auch der Versuchsaufbau dem vorliegenden sehr ähnlich war 
(Bestimmung von Laktat in der Nachbelastungsphase), konnte ein solcher Vergleich angestellt werden. Die gesunden Athleten zeigten durchweg signifikant höhere Laktatkonzentrationen nach Belastung als die Behindertensportler - Frauen wie Männer.

Über die exemplarische Darstellung der Laktatkonzentrationskurven einiger Athleten in Form von Exponentialfunktionen und Polynomen konnte gezeigt werden, dass Polynome den Verlauf des Laktats im Blut nach Belastung relativ gut abbilden können. Bei konstanter Leistung ähneln sich die Kurven der verschiedenen Messreihen eines Athleten.

Der Einsatz von Laktatkonzentrationskurven im Rahmen der Trainingsplanung könnte im Behindertenskisport - v.a. in den Disziplinen Slalom und Riesenslalom - ein geeignetes Mittel zur Leistungsdiagnose sein. Denn auf Grund der großen Heterogenität sind Vergleiche unter den Athleten nur begrenzt möglich. Stünden aber ausreichend Messreihen der einzelnen Athleten zur Verfügung, könnten weitere Informationen den Trainingsprozess betreffend gewonnen werden. Dies wäre über häufigere Untersuchungen - auch in den anderen Skidisziplinen - zu realisieren.

Denkbar wäre weiterhin eine Ergänzung der sportartunspezifischen Untersuchungen im Labor in der Vorbereitungsphase durch Messungen während eines Trainings in der Skihalle - auch in Form von Videoanalysen zur Kontrolle der technischen Umsetzung. Die Hinzunahme weiterer Untersuchungsparameter - wie der Herzfrequenz - ist dabei dringend anzuraten. 


\section{Anhang}

\subsection{System der Klassifizierung}

„Kategorie Sehbehindert mit 3 Klassen

Klasse B1 Total Sehbehindert

Klasse B2 Sehbehindert wenig Restsehvermögen

Klasse B3 Sehbehindert mehr Restsehvermögen

\section{$\underline{\text { Kategorie Stehend mit } 8 \text { Klassen }}$}

Klasse LW1 Doppel-Oberschenkelamputiert

Klasse LW2 Einseitig-Oberschenkelamputiert - Krückenskiläufer

Klasse LW3

Klasse LW3/1 Doppel-Unterschenkelamputiert oder inkomplette Querschnittlähmung

Klasse LW3/2 Cerebralparese

Klasse LW4 Einseitig-Unterschenkelamputiert - Prothesenskiläufer

Klasse LW5/7 Behinderung an beiden oberen Extremitäten

Klasse LW6/8 Behinderung an einer oberen Extremität - Einstockfahrer

Klasse LW9

Klasse LW9/1 Armbeh. und Oberschenkelamputiert oder schwere bis mäßige Hemiplegie

Klasse LW9/2 Armbeh. und Unterschenkelamputiert oder leichte Hemiplegie

\section{$\underline{\text { Kategorie Sitzend mit } 4 \text { Klassen }}$}

Klasse LW10

Klasse LW10/1 Monoskiläufer (ohne Rumpfmuskulatur)

Klasse LW10/2 Monoskiläufer (wenig Rumpfmuskulatur)

Klasse LW11 Monoskiläufer (mit Rumpfmuskulatur)

Klasse LW12

Klasse LW12/1 Monoskiläufer (niedrige inkomplette Querschnittlähmung)

Klasse LW12/2 Monoskiläufer (Doppeloberschenkelamputiert)“ 
(http:// www.skiteam-alpin.de 2008: Reglement: Klassen. Klassen im Behindertensport Ski Alpin).

\subsection{Der Monoski}

Querschnittgelähmte Skifahrer und jene mit beidseits lokalisierten Beinamputationen bedienen sich eines Monoskis.

Zur Konstruktion eines Monoskis sind Körpergröße und -gewicht sowie Länge von Ober- und Unterschenkeln wichtig, um die Breite und Tiefe sowie Winkel der Sitzschale festzulegen. Für den Bau der Schale sind weiterhin die Lokalisation der Querschnittlähmung bzw. Stabilisierungsmöglichkeiten der Rumpfmuskulatur sowie Bewegungsmöglichkeiten der Hüfte von Bedeutung. Bei einer im Thorakalmark lokalisierten Läsion kann der Rumpf in den unteren Anteilen nicht aktiv stabilisiert und verspannt werden; die Sitzschale des Monoskis muss dann höher gebaut werden, um diese Stabilisierung von außen zu gewährleisten. Die Rückenlehne ist durch einen Bauchgurt am Oberkörper befestigt. In der folgenden Abbildung ist das Gerüst des Monoskis zu erkennen:

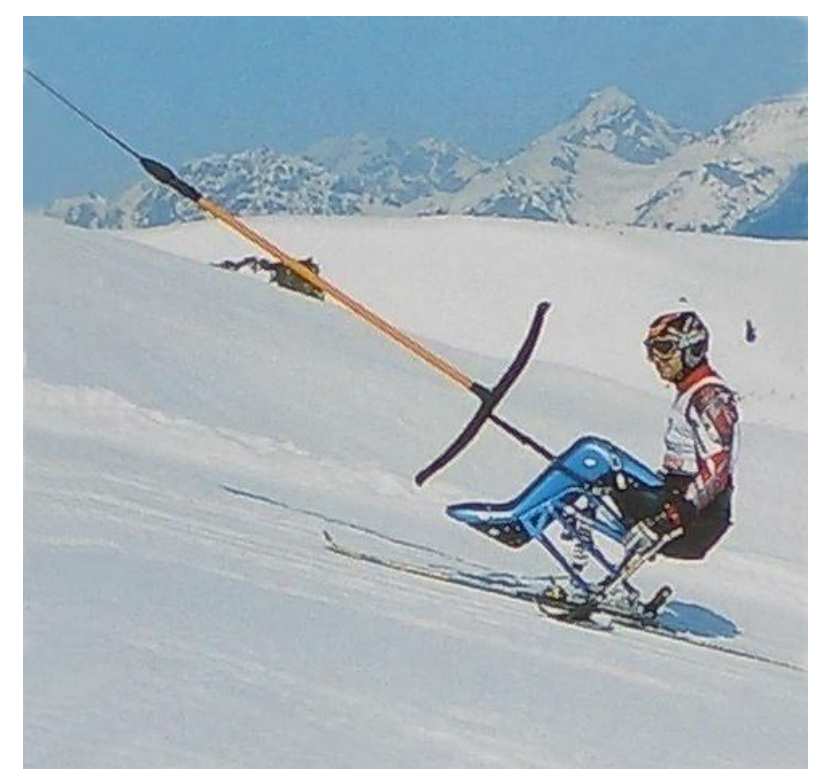

Abbildung 38: Monoski Liftfahrt (Quelle: PRASCHBERGER 2004)

In die Bindung des Skis ist eine Platte eingefasst; von dieser ausgehend ziehen Traversen zur Sitzschale. Zusätzlich eingebaute Stoßdämpfer ermöglichen eine Federung.

Die In Breiten- und Leistungssport eingesetzten Geräte unterscheiden sich in Material und Bauweise (Vgl. Wippermann 2007). 


\subsection{Definitionen}

\section{Leistungsdiagnostik}

„Lehre und Komplex von Verfahren der Leistungsdiagnose, d.h. der Erfassung und Beurteilung der sportlichen Leistungen und der aktuellen Leistungsfähigkeit... auf der Grundlage von Kennwerten, Kennlinien und Merkmalen des Leistungsvollzuges sowie von Kennwerten der wesentlichen personalen Leistungsvoraussetzungen“ (Schnabel G 1997, S. 49).

\section{Trainingssteuerung}

Diese ist definiert als ,gezielte Abstimmung aller kurz- und langfristigen Maßnahmen der Planung, Durchführung und Korrektur des Trainings zum Zwecke der Leistungsoptimierung“ (de Marées 2002, S. 479).

\section{Ergometrie}

„Messung physiologischer Größen während definierter muskulärer Belastung“ (de Marées 2002, S. 430).

\section{Trainingsstruktur}

„Aufbau, innerer Zusammenhang der wesentlichen Seiten, Bestandteile und Glieder des Trainingsprozesses, deren gesetzmäßiges Verhältnis und Zusammenwirken sowie ihre allgemeine Aufeinanderfolge.“ (Schnabel G und Thieß 1986)

\subsection{Lactate Scout - Technische Daten}

\section{„Gerätetyp: $\quad$ Lactate SCOUT}

Hersteller: $\quad$ SensLab GmbH, Bautzner Str. 67, D-04347 Leipzig

Messverfahren: $\quad$ Enzymatisch-amperometrische Bestimmung von Laktat in frischem Kapillarblut

Messbereich: $\quad 0,5-25,0 \mathrm{mmol} / \mathrm{l}$

Probevolumen: $\quad 0,5 \mu 1$

Messdauer: $\quad 15$ s bzw. 10 s (Lactate SCOUT bzw. Lactate SCOUT+)

Unpräzision: $\quad \leq 3,4 \mathrm{mmol} / \mathrm{l}: \pm 0,2 \mathrm{mmol} / \mathrm{l}$

$>3,4 \mathrm{mmol} / \mathrm{l}: \pm 6 \%$

Messtemperatur: $\quad \min .+5{ }^{\circ} \mathrm{C}, \max .+45^{\circ} \mathrm{C}$

Luftfeuchtigkeit: bis $85 \%$ relative Feuchtigkeit

Messwertspeicher: 250 Messwerte mit Zusatzparametern

Größe: $\quad 91 \times 55$ × $24 \mathrm{~mm}$ 
Gewicht: $\quad$ ca. $80 \mathrm{~g}$ mit Batterien

Anzeige: $\quad$ LC-Anzeige mit Symbolen

Stromversorgung: $2 \times 1,5 \mathrm{~V}$ AAA/LR03-Batterien“

(http:// www.senslab.de. Produkte. Lactate SCOUT. Technische Daten) 


\section{Literatur}

1. Aigner A, Muß N, Pfaller W (1990): Die anaerobe Schwelle von Rollstuhlfahrern. Dtsch Z Sportmed $\underline{41(9)}, 312-315$

2. Bachl N, Baron R, Smekal G, Tschan H: Validität sportartspezifischer Leistungsdiagnostik; in: Clasing D, Weicker H, Böning D: Stellenwert der Laktatbestimmung in der Leistungsdiagnostik, Gustav Fischer Verlag, Stuttgart 1994, 165 174

3. Berghold F, Bachl N, Hamar D, Erd E (1984): Metabolische Beanspruchungen im Tourenskilauf. Dtsch Z Sportmed 35 (12), 407-414

4. Braumann K-M, Busse M, Maassen N (1987): Zur Interpretation von LaktatLeitungskurven. Leistungssport 17 (4), 35-38

5. Clasing D: Leistungsdiagnostik - (sportartspezifische) Tests im Labor; in: Clasing D, Weicker H, Böning D: Stellenwert der Laktatbestimmung in der Leistungsdiagnostik, Gustav Fischer Verlag, Stuttgart 1994, 221-227

6. De Marées H : Sportphysiologie. 9. Auflage, Verlag Sport und Buch Strauß, Köln 2002

7. Fecht R: Gelände, Schneebeschaffenheit, Wetter und Streckenführung als Bedingungsfaktoren für Bewegungshandlungen im Skilauf; in: Skilauf in der Sportlehrerausbildung - Materialien für die Ausbildung im Skilauf, hrsg. v. Arbeitsgemeinschaft Ausbildung im Skilauf an Hochschulen, Heft 4, Esslingen 1980, 8595

8. Fendt E (2002) : Monoski-Lehrplan, (im Druck)

9. Heck H: Energiestoffwechsel und medizinische Leistungsdiagnostik. Verlag Karl Hofmann, Schorndorf 1990

10. Heck H, Hess G, Mader A (1985): Vergleichende Untersuchung zu verschiedenen LaktatSchwellenkonzepten. Dtsch Z Sportmed $\underline{36}$ (1), $19-25 ; \underline{36(2)}, 40-52$

11. Hintzy F, Grenier J, Coulmy N: Energetic and respective part of lactic and aerobic sources over a giant slalom in young ski racers; in: $14^{\text {th }}$ annual Congress of the European College of Sport Science. Book of Abstracts. Oslo/Norway, June 24-27, 2009, 500

12. Hollmann W: Kardio- Pulmonale und metabolische Reaktionen und Anpassungserscheinungen sowie Belastbarkeit beim Skisport im Erwachsenen- und Seniorenalter; in: Medizinische Probleme des Skisports. (Schriftenreihe des Deutschen 
Skiverbandes, hrsg. v. Jäger M, Ulmrich E, Heft 13), 1. Auflage, DSV München 1982, 189-200

13. http://www.dbs-npc.de. Deutscher Behindertensportverband e.V. - National Paralympic Committee Germany. (Letzter Zugriff: 06.03.2011)

14. http://www.dlr.de. Deutsches Zentrum für Luft- und Raumfahrt in der HeimholtzGemeinschaft, Göttingen. (Letzter Zugriff: 20.04.2011)

15. http://www.skiteam-alpin.de. Förderverein Skiteam alpin der Behinderten e.V. - Deutsches Paralympic Skiteam alpin (2008): Reglement. Handycap-System. Das Handicap-System. (Letzter Zugriff: 06.03.2011)

16. http://www.skiteam-alpin.de. Förderverein Skiteam alpin der Behinderten e.V. - Deutsches Paralympic Skiteam alpin (2008): Reglement: Die Klassifizierung. (Letzter Zugriff: 02.03.2011)

17. http://www.skiteam-alpin.de. Förderverein Skiteam alpin der Behinderten e.V. - Deutsches Paralympic Skiteam alpin (2008): Reglement: Klassen. Klassen im Behindertensport Ski Alpin. (Letzter Zugriff: 29.03.2011)

18. http://www.senslab.de. SensLab Gesellschaft zur Entwicklung und Herstellung bioelektrochemischer Sensoren mbH Leipzig: Produkte. Lactate SCOUT. Technische Daten. (Letzter Zugriff: 31.03.2011)

19. Jonath U, Krempel R: Konditionstraining - Training Technik Taktik, Rowohlt Verlag, Reinbek 1981

20. Keul J: Die Anpassung der Energiebereitstellung im Muskel an körperliche Arbeit; in: Keul J, Doll E, Keppler D: Muskelstoffwechsel. Die Energiebereitstellung im Skelettmuskel als Grundlage seiner Funktion. (Wissenschaftliche Schriftenreihe des Deutschen Sportbundes) Johann Ambrosius Barth, München 1969, 193-200

21. Keul J, Huber G, Kindermann W (1975): Unterschiedliche Wirkung des Skilanglaufes und des Skiabfahrtslaufes auf Kreislauf und Stoffwechsel. Sportarzt + Sportmedizin 26 (3), 49-58

22. Keul J, Berg A, Huber G, Lehmann M, Dickhuth H-H: Kardiozirkulatorische und metabolische Veränderungen bei alpinen und nordischen Skiläufern; in: Medizinische Probleme des Skisports. (Schriftenreihe des Deutschen Skiverbandes, hrsg. v. Jäger M, Ulmrich E, Heft 13), 1. Auflage, DSV München 1982, 124- 145

23. Kindermann W, Keul J (1977a): Anaerobe Kapazität bei verschiedenen körperlichen Belastungsformen; in: Deutscher Sportbund. Informationen zum Training. 
Ausdauertraining - Stoffwechselgrundlagen und Steuerungsansätze. Beiheft zu Leistungssport. Verlag Bartels \& Wernitz, Berlin 1977, 80-92

24. Kindermann W, Keul J (1977b): Anaerobe Energiebereitstellung im Hochleistungssport. Die Bedeutung der metabolischen Azidose unter physiologischen und pathologischen Bedingungen. 1. Auflage (Wissenschaftliche Schriftenreihe des Deutschen Sportbundes, Band 13), Hofmann, Schorndorf 1977

25. Klinke R, Silbernagl S: Lehrbuch der Physiologie. 4. Auflage Thieme Verlag, Stuttgart 2003

26. Koch HJ, Rachka C (1999): Empirische und mechanistische Auswertungsmodelle für Laktatkonzentrationskurven im Dauerleistungstest. Nagoya J Med Sci $\underline{62}$, 127-134

27. Lochner S, Rettenmeier A, Barwich D, Weicker H (1983): Stoffwechseluntersuchungen an Skilehrern im Riesentorlauf und Langlauf. Dtsch Z Sportmed 34 (4), 109-122

28. Löffler G: Basiswissen Biochemie mit Pathobiochemie. 4. Auflage, Springer Verlag, Berlin 2001

29. Mayr M: Untersuchung der Belastungsparameter bei Freeskiern. Sport Diss. Wien, 2008

30. Mester J, de Marées H (1981): Zur Kontrolle der physischen Beanspruchung beim alpinen Skilauf. Sportwissenschaft 11 (1), 75-87

31. Neumann G, Gottschalk K: Ausgewählte sportmedizinische Beiträge zur Leistungsentwicklung in der Sportartengruppe Ausdauer. 2. Auflage, DHfK Leipzig 1982

32. Pansold B: Beurteilung der aeroben und anaeroben Leistungsvoraussetzungen in der Leistungsdiagnostik und Trainingssteuerung durch Bestimmung der Laktatkonzentration im Blut; in: Empfehlungen zum Einsatz der Parameter Laktat und Kreatinkinase in der Leistungsdiagnostik. Zur sportmedizinischen Betreuung. (Schriftenreihe Sportmedizinischer Dienst, Heft 8), Sportmedizinischer Dienst Kreischa 1983, 4-22

33. Pansold B, Zinner J: Die Laktat-Leistungskurve - ein Analyse- und Interpretationsmodell der Leistungsdiagnostik im Schwimmen; in: Clasing D, Weicker H, Böning D: Stellenwert der Laktatbestimmung in der Leistungsdiagnostik, Gustav Fischer Verlag, Stuttgart 1994, 47-64

34. Pessenhofer H, Schwaberger G: Stellenwert der der Laktatbestimmung in der Leistungsdiagnostik; in: Clasing D, Weicker H, Böning D: Stellenwert der Laktatbestimmung in der Leistungsdiagnostik, Gustav Fischer Verlag, Stuttgart 1994, 153164 
35. Praschberger A Rolltechnik und Sport GmbH, schriftliche Mitteilung, Ebbs 2004

36. Pschyrembel. Klinisches Wörterbuch. S.v. „Behinderung“. 256. Auflage, De Gruyter, Berlin 1990, 190

37. Pschyrembel. Klinisches Wörterbuch. S.v. „Behinderung“. 261. Auflage. De Gruyter, Berlin 2007, 221

38. Röder Y: Metabolische und kardiale Parameter beim alpinen Skilauf - Komparative Analysen zur Belastung von Freizeit-, Seniorenskiläufern und aktiven Skilehrern beim Feldtest mit differierenden Skitaillierungen und beim Labortest auf dem Abfahrtssimulator. SoWi. Diss. Konstanz 2001 (Shaker, Aachen 2002)

39. Saibene F, Cortili G, Gavazzi P, Magistri P (1985): Energy sources in alpine skiing (giant slalom). Eur J Appl Physiol 53, 312-316

40. Schnabel A, Kindermann W, Keul J, Schmitt W-M (1979): Beurteilung der anaeroben Ausdauer („Stehvermögen“) im Labor. Leistungssport 9 (6), 503-507

41. Schnabel G: Trainingswissenschaft: Leistung - Training - Wettkampf. 2. Auflage, SVB Sportverlag, Berlin 1997

42. Schnabel G, Thieß G: Grundbegriffe des Trainings. S.v. „Trainingsstruktur“. 1. Auflage, Sportverlag, Berlin 1986

43. Schnabel G, Thieß G (Ltg.): Leistungsfaktoren in Training und Wettkampf. 1. Auflage, Sportverlag, Berlin 1987

44. Schnabel G, Harre H-D, Krug J: Trainingslehre - Trainingswissenschaft, Meyer\&Meyer Verlag, Aachen 2008

45. Simon G, Berg A, Dickhuth H-H, Simon-Alt A, Keul J (1981): Bestimmung der anaeroben Schwelle in Abhängigkeit vom Alter und von der Leistungsfähigkeit. Dtsch Z Sportmed $\underline{32(1)}, 7-14$

46. Sozialgesetzbuch IX, §2 Absatz 1

47. Stegemann J : Leistungsphysiologie. 4. überarb. Auflage, Georg Thieme Verlag, Stuttgart 1991

48. Temme B: Laktatkinetik und Catecholaminverhalten im alpinen Skirennsport. Med. Diss Freiburg 2001

49. Veicsteinas A, Ferretti G, Margonato V, Rosa G, Tagliabue D (1984): Energy cost of and 
energy sources for alpine skiing in top athletes. J Appl Physiol: Respiratory,

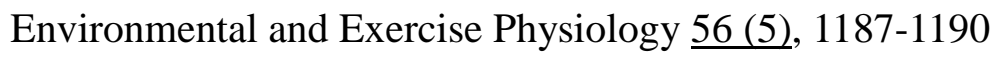

50. Weicker H: Interaktion zwischen aerober und anaerober Energieproduktion, Laktatproduktion, Release und Elimination; in: Clasing D, Weicker H, Böning D: Stellenwert der Laktatbestimmung in der Leistungsdiagnostik, Gustav Fischer Verlag, Stuttgart 1994, 11-25

51. Weineck J: Optimales Training - leistungsphysiologische Trainingslehre unter besonderer Berücksichtigung des Kindes- und Jugendtrainings. 15. Auflage, Spitta Verlag, Balingen 2007

52. Wippermann F: Behindertensport im Bereich Ski alpin - Vermittlung des Skifahrens im Breitensport am Beispiel des Lehrplans Monoski. Magisterarbeit, Göttingen 2007

53. Zimmer M (2007): Orthopädische Aspekte des Behindertensports; in: Dickhuth H-H: Sportmedizin für Ärzte, Deutscher Ärzteverlag, Köln, 451-457 


\section{Danksagung}

Ich danke Herrn Prof. Dr. Dr. A. Niklas für die Überlassung des Themas, seine sehr engagierte Betreuung und wertvollen inhaltlichen Anregungen. Bei Frau Dr. U. HillmerVogel und Herrn Dr. H. Stinus bedanke ich mich für die Unterstützung und anregende Hinweise.

Frau Dr. K. Lange und den Mitarbeitern der Abteilung Medizinische Statistik danke ich für die Beratung.

Insbesondere möchte ich mich bei den Probanden der Studie für ihr Engagement bedanken. 